\title{
Numerical treatment and Global Error Estimation of a MHD Flows of an Oldroyd 6-Constant Nano-Fluid through a non- Darcy Porous medium with Heat and Mass Transfer
}

\begin{abstract}
Abeer A. Shaaban
Department of Mathematics, Faculty of Education, Ain Shams University, Roxy, Cairo, Egypt

Department of Management Information Systems, Faculty of Business Administration in Rass, Qassim University, Qassim, KSA

Abstract - Explicit Finite-Difference method was used to obtain the solution of the system of the non-linear ordinary differential equations which transform from the non-linear partial differential equations. These equations describe the steady magneto-hydrodynamic flow of an oldroyd 6-constant non-Newtonian nano-fluid through a non-Darcy porous medium with heat and mass transfer. The numerical formula of the velocity, the temperature, the concentration, and the nanoparticles concentration distributions of the problem were illustrated graphically. The effect of Darcy number $D_{a}$, Forchheimer number $F_{s}$, magnetic field parameter $M$, local temperature Grashof number $G_{r}$, local nanoparticle Grashof $B_{r}$, Prandtl number $P_{r}$, Dufour number $N_{d}$, Brownian motion parameter $N_{b}$, Thermophoresis parameter $N_{t}$, Lewis number $L_{e}$, Sort number $L_{d}$, Chemical reaction parameter $R_{c}$, and Chemical reaction order $m$ on those formula were discussed at the values of material parameters $\left(\alpha_{1}=8, \alpha_{2}=2\right)$ specially in the case of pure Coutte flow. Then, the effects of modified pressure gradients on those formulas were discussed in the case of pure Poiseuille flow and the generalized Couette flow. Also, an estimation of the global error for the numerical values of the solutions is calculated by using Zadunaisky technique.
\end{abstract}

Keywords - Finite-difference method, Heat and Mass transfer, MHD flows, Non-Darcy Porous medium, Oldoryd 6Constant non-Newtonian nano-Fluid.

\section{NOMENCLATURE}

\begin{tabular}{|c|c|c|c|}
\hline$A$ & Chemical Reaction rate constant & $R_{c}$ & Chemical reaction parameter, defined by Eq. (42) \\
\hline$B_{r}$ & $\begin{array}{l}\text { Local nanoparticle Grashof number, } \\
\text { defined by Eq. ( } 42 \text { ) }\end{array}$ & $S$ & The dimensionless concentration \\
\hline$c^{*}$ & Forchheimer number & $t$ & The time \\
\hline$C$ & The concentration of the fluid & $T$ & The fluid temperature \\
\hline$C_{1}$ & The concentration at lower plate $(y=0)$ & $T_{1}$ & The temperature at lower plate $(y=0)$ \\
\hline$C_{2}$ & The concentration at upper plate $(y=H)$ & $T_{2}$ & The temperature at upper plate $(y=H)$ \\
\hline$C_{s}$ & Nanoparticle susceptibility & $\underline{V}$ & The velocity vector \\
\hline$D_{\mathrm{a}}$ & Darcy number, defined by Eq. (42) & & \\
\hline $\begin{array}{c}D \\
\text { в }\end{array}$ & Brownian diffusion coefficient & & \\
\hline $\begin{array}{c}D \\
\mathrm{~T}\end{array}$ & Thermophoretic diffusion coefficient & & Greek symbols \\
\hline$\underline{E}$ & Electrical field & $\hat{\phi}$ & The nanoparticles phenomena \\
\hline$\underline{F}$ & The external force & $\Phi$ & The dissipation function \\
\hline$F_{s}$ & Forchheimer number, defined by Eq. (42) & $\phi$ & The dimensionless nanoparticles \\
\hline$G$ & Gravitational acceleration & $\theta$ & The dimensionless temperature \\
\hline$G_{r}$ & $\begin{array}{l}\text { Local temperature Grashof number, } \\
\text { defined by Eq. ( } 42 \text { ) }\end{array}$ & $\alpha_{1}, \alpha_{2}$ & The non-Newtonian parameters, defined by Eq. (36) \\
\hline$\underline{H}$ & The magnetic field & & \\
\hline$\underline{J}$ & The current density & $\nabla$ & Gradient operator \\
\hline$k$ & Thermal conductivity & $\nabla^{2}$ & Laplacian operator \\
\hline
\end{tabular}




\begin{tabular}{|c|c|c|c|}
\hline$k^{*}$ & Permeability constant & $\mu$ & the dynamic viscosity of fluid \\
\hline$K_{T}$ & Thermal diffusion ratio & $\mu_{e}$ & the magnetic permeability \\
\hline$L_{e}$ & Lewis number, defined by Eq. (42) & $v$ & The kinematic viscosity $\left(\mu / \rho_{f}\right)$ \\
\hline$L_{d}$ & Sort number, defined by Eq. (42) & $c_{p}$ & The specific heat capacity at constant pressure \\
\hline$M$ & Chemical Reaction order & $\rho_{f}$ & The density of the fluid \\
\hline$M$ & $\begin{array}{l}\text { Magnetic field parameter, defined by Eq. } \\
(42)\end{array}$ & $\rho_{p}$ & The density of the particle \\
\hline$N_{b}$ & $\begin{array}{l}\text { Brownian motion parameter, defined by Eq. } \\
(42)\end{array}$ & $(\rho c)_{f}$ & heat capacity of the fluid \\
\hline$N_{d}$ & Dufour numbe, defined by Eq. (42) & $(\rho c)_{p}$ & effective heat capacity of the nanoparticle material \\
\hline$N_{t}$ & $\begin{array}{l}\text { The thermophoresis parameter, defined by } \\
\text { Eq. }(42)\end{array}$ & $\sigma$ & Electrical conductivity of the fluid \\
\hline$P$ & The fluid pressure & $\tau$ & The Cauchy Stress tensor \\
\hline$P_{r}$ & Prandtl number, defined by Eq. (42) & $\alpha_{T}, \alpha_{C}$ & $\begin{array}{l}\text { Volumetric thermal and solute expansion coefficients of the } \\
\text { base fluid }\end{array}$ \\
\hline
\end{tabular}

\section{INTRODUCTION}

The study of non-Newtonian fluids has gained much attention recently in view of its promising applications in engineering and industry. Such fluids exhibit a non-linear relationship between the stresses and the rate of strain. Due to non-linear dependence, the analysis of the behavior of the non-Newtonian fluids tends to be much more complicated and subtle in comparison with that of Newtonian fluids. Flow of fluids with complex microstructure (e. g. molten polymer, polymer solutions, blood, paints, greases, oils, ketchup, etc.) cannot be described by a single model of non-Newtonian fluids. Many models that exist are based either on natural modifications of established macroscopic theories or molecular considerations. In general, the equations of motion for non-Newtonian fluids are of higher order than the Navier-Stokes equations and thus one need conditions in addition to the usual adherence boundary condition [28].

Guillope and Saut [2] has established existence results for some shearing motions of viscoelastic fluids of Oldroyd type. Some exact solutions of an Oldroyd 3-constant fluid are studied in [4], [5], [11], [13]. Baris [21] investigated the steady flow of an Oldroyd 6-constant fluid between intersecting planes using the series expansion method. Hayat et al. [23] studied the Couette and Poiseuille flows of an Oldroyd 6-constant fluid with magnetic field by using the Homotopy analysis method. Hayat et al. [25] studied the steady flow of a magneto-hydrodynamic Oldroyd 6-constant fluid by the motion of an infinite plate using the Homotopy analysis method. Wang et al. [30] investigated the non-linear magnetohydrodynamic problems of an Oldroyd 6-constant fluid by using analytical method and the finite-difference discretization method. Hayat et al. [24] studied the effect of the slip condition on flows of an Oldroyd 6-constant fluid. Rana et al. [14] studied the Hall effects on hydromagnetic flow of an Oldroyd 6-constant fluid between concentric cylinders by the finite difference method. Hayat et al. [26] investigated the exact solution of a thin film flow of an Oldroyd 6-constant fluid over a moving belt by the Homotopy perturbation.

Investigation of nanofluid flow has received special focus in the past due to its relevance in numerous industrial applications. The researchers not only discovered unexpected thermal properties of nanofluids but also proposed mechanisms behind the enhanced thermal properties of nanofluids and thus identified unusual opportunities to develop them as next generation coolants for computers and safe coolants for nuclear reactors. A combination of nanofluid with biotechnological components can provide potential applications in agriculture, pharmaceuticals and biological sensors. Various types of nanomaterials including nanoparticles, nanowires, nanofibers, nanostructures, and nanomachines are used in biotechnological applications. The commercialization of nano-biotechnological products seems to have a potential future and within next a few years many new products of this nature are likely to be used. Nano and micro-fluidics is a new area which has potential for engineering applications, especially for the development of new biomedical devices and procedures [1]. 
The magnetic nanofluids possess both liquid and magnetic properties. These fluids have key importance in modulators, optical switches, optical gratings and tunable optical fiber filters. The magnetic nanoparticles are significant in medicine, construction of loud speakers, sink float separation, cancer therapy and tumor analysis. Nowadays, the sustainable energy generation is one of the most important issues across the globe. Perhaps the solar energy is also one of the best sources of renewable energy with minimal environment impact. Solar power is a direct way obtaining heat, water, and electricity from the nature. Researchers concluded that heat transfer and solar collection processes can be improved through the addition of nanoparticles in the fluids. Fluid heating and cooling are required in many industrial fields such as power manufacturing and transportation. Effective cooling techniques are needed for cooling any sort of high energy device. Common heat transfer fluids such as ethylene glycol, water and engine oil have limited heat transfer capabilities due to their low heat transfer properties and thus cannot meet the modern cooling requirements [1].

The terminology of nanofluid was first used by Choi [22] when he experimentally discovered a significant improvement in the heat transfer performance through the addition of small amount nanometer sized particles in the base fluids. This addition also causes scattering of the incident radiation which allows a deeper absorption within the fluid. Recently, Trieb and Nitsch [3] proposed the idea of solar thermal collectors by using nanofluids to directly absorb the solar radiation.

The phenomenon of thermal conductivity enhancement by dispersing nanoparticles in the liquid was also observed by Masuda et al. [7]. Buongiorno [9] recognized that the two main effects namely the Brownian motion and thermophoretic diffusion of nanoparticles contribute to the massive increase in the thermal conductivity of the liquids. He also proposed the modifications in the transport equations due to these effects.

Vajravelu et al. [12] investigated heat and mass transfer properties of three-layer fluid flow in which nano-fluid layer is squeezed between two clear viscous fluid. Farooq et al. [27] studied heat and mass transfer of two-layer flows of third-grade nano-fluids in a vertical channel. Abou-zeid et al. [16] obtained numerical solutions and Global error estimation of natural convection effects on gliding motion of bacteria on a power-law nano-slime through a non-Darcy porous medium. El-Dabe et al. [17] investigated magneto-hydrodynamic non-Newtonian nano-fluid flow over a stretching sheet through a non-Darcy porous medium with radiation and chemical reaction.

Magneto-hydrodynamic (MHD) is concerning the mathematical and physical scaffold that introduces magnetic-dynamics in electrically conducting fluids (e.g. in plasmas and liquid metals ). The applications of Magneto-hydrodynamic incompressible viscous flow in science and engineering involving heat and mass transfer under the influence of chemical reaction is great importance to many areas of science and engineering. This frequently occurs in agriculture, engineering, plasma studies and petroleum industries [15].

Flow through porous media plays an important role in countless practical applications such as ground water flows, enhanced oil recovery processes, contamination of soils by hazardous wastes, pollution movement [20]. An understanding of the dynamics of fluids in a porous medium is of principal interest because these flows are quite prevalent in nature. Such flows have attracted the attention of number of scholars due to their applications in many branches of science and technology, viz. in the fields of agricultural engineering to study the underground water resources; seepage of water in river beds; in petroleum technology to study the movement of natural gas, oil, and water through the oil reservoirs. In the widely used continuum approach to transport processes in a porous media, the differential equation governing the macroscopic fluid motion is based on the experimentally established Darcy law [8], which accounts for the drag exerted on the fluid by porous medium [29].

The objective of this work is to investigate the numerical solution by using Explicit Finite Difference method [6] for the system of non-linear differential equations which arises from magneto-hydrodynamic flows of an Oldroyd 6-constant Nanofluid with a magnetic field through a non-Darcy porous medium with heat and mass transfer. We obtained the distributions of the velocity, the temperature, the concentration, and the Nanoparticles. Numerical results are found for different values of various non-dimensional parameters in the case of pure Coutte flow $\left(\mathrm{U}_{0}=1, \frac{\mathrm{d} \widehat{\mathrm{P}}}{\mathrm{dx}}=0\right)$. The effects of modified pressure gradients on those formulas were discussed in the case of pure Poiseuille flow and the generalized Couette flow. The results are shown graphically and discussed in detail. Also, the global error estimation for the error propagation is obtained by Zadunaisky technique [19].

\section{FLUID MODEL}

For an Oldroyd 6-constant fluid, the Cauchy stress tensor $\tau$ is given by [30] 


$$
\begin{aligned}
& \tau=-P I+S^{*} \\
& S^{*}+\lambda_{1} \frac{D S^{*}}{D t}+\frac{\lambda_{3}}{2}\left(S^{*} A_{1}+A_{1} S^{*}\right)+\frac{\lambda_{5}}{2}\left(\operatorname{tr} S^{*}\right) A_{1}=\mu\left[A_{1}+\lambda_{2} \frac{D A_{1}}{D t}+\lambda_{4} A_{1}^{2}\right] .
\end{aligned}
$$

Where,

$$
A_{1}=L+L^{T} \quad, \quad L=\operatorname{grad} V
$$

$-P I$ is the indeterminate part of the stress due to the constraint of incompressibility, $\mathrm{S}^{*}$ is the extra stress tensor, $A_{1}$ is the first Rivlin-Ericksen tensor, $\mathrm{L}$ is the velocity gradient, $\underline{V}$ is the velocity vector, and $\mu, \lambda_{i}(i=1,2, \ldots \ldots, 5)$ are the six material constants.

The contra variant convected derivative $\frac{D}{D t}$ satisfying the principle of material frame in difference in terms of the material $\frac{d}{d t}$ is defined by the following equation

$$
\frac{D S^{*}}{D t}=\frac{d S^{*}}{d t}-L S^{*}-S^{*} L^{T}
$$

in which the superscript $\mathrm{T}$ is used for the transpose operation.

It should be noted that when $\lambda_{i}=0(i=1,2,3,4,5)$ the model reduces to the classical linearly viscous model of Newtonian fluid. For $\lambda_{i}=0(i=3,4,5)$ a 3 -constant model of an Oldroyd-B fluid is described, while for $\lambda_{i}=0(i=2,3,4,5)$ a Maxwell model is formulated. For $\lambda_{i}=0(i=1,3,4,5)$ the model describes a second-grade fluid.

\section{MATHEMATICAL FORMUlation}

We consider magnetohydrodynamic flows of an Oldroyd 6-constant Nano-fluid through a non-Darcy porous medium between two parallel plates (see FIG. 1). We take in our consideration the presence of heat and mass transfer with chemical reaction.

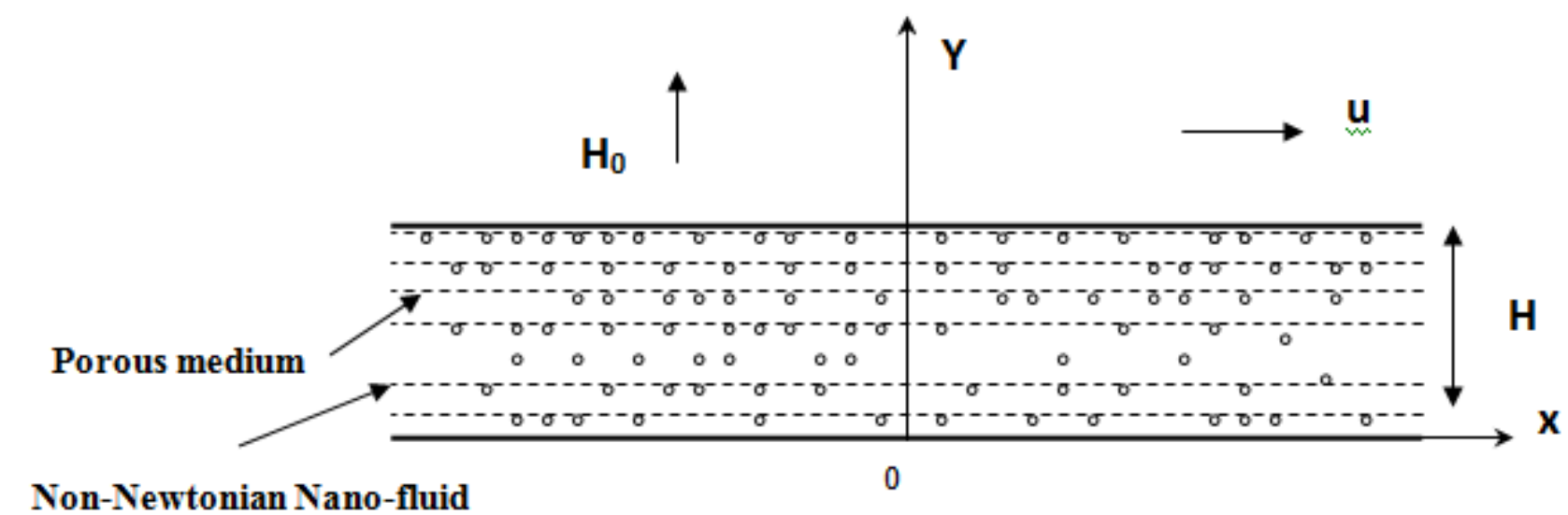

\section{(Oldroyed 6-Constant Nano-fluid)}

\section{FIGURE 1: MAGNETOHYDRODYNAMIC FLOWS OF AN OLDROYD 6-CONSTANT NANO-FLUID THROUGH A NON- DARCY POROUS MEDIUM BETWEEN TWO PARALLEL PLATES}

\section{BASIC EQUATIONS}

The basic equations governing the flow of an incompressible fluid are the following equations [10, 18],

The continuity equation

$$
\nabla \cdot \underline{V}=0 \quad,
$$

The momentum equation

$$
\rho_{f} \frac{d \underline{V}}{d t}=\nabla \cdot \tau+\mu_{e}(\underline{J} \wedge \underline{H})-\rho_{f} c^{*} \underline{V}|\underline{V}|-\frac{\mu}{k^{*}} \underline{V}+\underline{F},
$$


The energy equation

$$
\left(\rho c_{p}\right)_{f}\left[\frac{d T}{d t}\right]=k \nabla^{2} T+\Phi+\left(\rho c_{p}\right)_{p}\left[D_{B}(\nabla T . \nabla \hat{\phi})+\frac{D_{T}}{T_{1}}(\nabla T)^{2}\right]+\frac{\rho_{f} D_{B} \kappa_{T}}{C_{S}}
$$

The concentration equation

$$
\frac{d C}{d t}=D_{B} \nabla^{2} C+\frac{D_{T} \kappa_{T}}{T_{1}} \nabla^{2} T-A\left(C-C_{2}\right)^{m} \quad,
$$

The nanoparticles concentration equation

$$
\frac{d \widehat{\phi}}{d t}=D_{B} \nabla^{2} \hat{\phi}+\frac{D_{T}}{T_{2}} \nabla^{2} T
$$

Where, $\frac{d}{d t}=\left[\frac{\partial}{\partial t}+(\underline{V} . \nabla)\right]$ denotes the material time derivative, $\underline{J}=\sigma\left(\underline{E}+\mu_{e} \underline{V} \wedge \underline{H}\right)$, and $\underline{E}=0$.

For a simple one-dimensional shearing flow considered in this work, the extra stress tensor and the velocity are of the form

$$
S(y, t)=\left(\begin{array}{ccc}
S_{x x} & S_{x y} & S_{x z} \\
S_{y x} & S_{y y} & S_{y z} \\
S_{z x} & S_{z y} & S_{z z}
\end{array}\right), \quad V(y, t)=\left(\begin{array}{c}
u(y, t) \\
0 \\
0
\end{array}\right)
$$

Also, the constant magnetic field, the temperature, the concentration, and the nanoparticles are of the form

$$
\underline{H}=\left(0, H_{0}, 0\right), T=T(y, t), C=C(y, t), \hat{\phi}=\hat{\phi}(y, t) .
$$

For steady motion, it is clear that with the simplified dependence (10-11), the continuity equation (5) is satisfied identically, and equations (1-3) and (6) become,

$$
\begin{aligned}
& \frac{\partial P}{\partial x}=\frac{d S_{x y}}{d y}-\sigma \mu_{e}^{2} H_{0}^{2} u-\rho c^{*} u^{2}-\frac{\mu}{\kappa^{*}} u+\rho_{f} g \alpha_{T}\left(T-T_{2}\right)+\rho_{f} g \alpha_{C}\left(C-C_{2}\right), \\
& \frac{\partial P}{\partial y}=\frac{d S_{y y}}{d y}, \\
& \frac{\partial P}{\partial z}=\frac{d S_{z y}}{d y}, \\
& S_{x x}+\left(\lambda_{3}-2 \lambda_{1}\right) S_{x y} \frac{d u}{d y}=\mu\left(\lambda_{4}-2 \lambda_{2}\right)\left(\frac{d u}{d y}\right)^{2}, \\
& S_{x y}-\lambda_{1} S_{y y} \frac{d u}{d y}+\left(\frac{\lambda_{3}+\lambda_{5}}{2}\right)\left(S_{x x}+S_{y y}\right) \frac{d u}{d y}+\frac{\lambda_{5}}{2} S_{z z} \frac{d u}{d y}=\mu\left(\frac{d u}{d y}\right), \\
& S_{z x}+\left(\frac{\lambda_{3}-2 \lambda_{1}}{2}\right) S_{z y} \frac{d u}{d y}=0 \\
& S_{y y}+\lambda_{3} S_{x y} \frac{d u}{d y}=\mu \lambda_{4}\left(\frac{d u}{d y}\right)^{2}, \\
& S_{z y}+\frac{\lambda_{3}}{2} S_{z x} \frac{d u}{d y}=0 \\
& S_{z z}=0
\end{aligned}
$$

by addition of two equations (15) and (18) we have,

$$
S_{x x}+S_{y y}=2 \mu\left(\lambda_{4}-\lambda_{2}\right)\left(\frac{d u}{d y}\right)^{2}-2\left(\lambda_{3}-\lambda_{1}\right) S_{x y} \frac{d u}{d y}
$$

from equations (17) and (19) we get 


$$
S_{z x}=S_{z y}=0
$$

Substituting (18) - (22) in (14) and (16) we have

$$
\begin{aligned}
& \frac{\partial P}{\partial z}=0 \\
& S_{x y}=\frac{\left(\mu \frac{d u}{d y}+\mu \alpha_{1}\left(\frac{d u}{d y}\right)^{3}\right)}{\left(1+\alpha_{2}\left(\frac{d u}{d y}\right)^{2}\right)}
\end{aligned}
$$

where,

$$
\begin{aligned}
& \alpha_{1}=\lambda_{1} \lambda_{4}-\left(\lambda_{3}+\lambda_{5}\right)\left(\lambda_{4}-\lambda_{2}\right) \\
& \alpha_{2}=\lambda_{1} \lambda_{3}-\left(\lambda_{3}+\lambda_{5}\right)\left(\lambda_{3}-\lambda_{1}\right)
\end{aligned}
$$

Defining the modified pressure

$$
\hat{P}=P-S_{y y},
$$

and using (12) - (14) and (24) we arrive at

$$
\begin{aligned}
& \frac{\partial \hat{P}}{\partial x}=\frac{d}{d y}\left[\frac{\left(\mu \frac{d u}{d y}+\mu \alpha_{1}\left(\frac{d u}{d y}\right)^{3}\right)}{\left(1+\alpha_{2}\left(\frac{d u}{d y}\right)^{2}\right)}\right]-\sigma \mu_{e}^{2} H_{0}^{2} u-\rho c^{*} u^{2}-\frac{\mu}{\kappa^{*}} u+\rho_{f} g \alpha\left(T-T_{2}\right)+\rho_{f} g \alpha\left(C-C_{2}\right), \\
& \frac{\partial \hat{P}}{\partial y}=\frac{\partial \hat{P}}{\partial z}=0
\end{aligned}
$$

The dissipation function $\Phi$ can be written as follows

$$
\Phi=\tau_{i j} \frac{\partial V_{i}}{\partial x_{j}}
$$

By using the definitions (10) we have,

$$
\Phi=\mathrm{S}_{x y} \frac{d u}{d y}
$$

Then, equations (7 - 9) can be written as follows:

$$
\begin{aligned}
& \kappa \frac{d^{2} T}{d y^{2}}+\frac{\rho_{f} D_{B} \kappa_{T}}{C_{S}} \frac{d^{2} C}{d y^{2}}+\left(\rho c_{p}\right)_{p}\left[D_{B} \frac{d T}{d y} \frac{d \widehat{\phi}}{d y}+\frac{D_{T}}{T_{1}}\left(\frac{\mathrm{d} T}{d y}\right)^{2}\right]+\left[\frac{\left(\mu \frac{d u}{d y}+\mu \alpha_{1}\left(\frac{d u}{d y}\right)^{3}\right)}{\left(1+\alpha_{2}\left(\frac{d u}{d y}\right)^{2}\right)}\right] \frac{d u}{d y}=0 \\
& D_{B} \frac{d^{2} C}{d y^{2}}+\frac{D_{T} \kappa_{T}}{T_{1}} \frac{d^{2} T}{d y^{2}}-A\left(C-C_{2}\right)^{m}=0 \\
& D_{B} \frac{d^{2} \widehat{\phi}}{d y^{2}}+\frac{D_{T}}{T_{2}} \frac{d^{2} T}{d y^{2}}=0
\end{aligned}
$$

The boundary conditions of the problem are given by:

$$
\begin{aligned}
& u=0, T=T_{1}, C=C_{1}, \hat{\phi}=\hat{\phi}_{1} \quad \text { at } \quad y=0, \\
& u=u_{0}, T=T_{2}, C=C_{2}, \hat{\phi}=\hat{\phi}_{2} \quad \text { at } \quad y=H .
\end{aligned}
$$

We shall now write the field equations (28), $(32-34)$ and the boundary conditions (35) in terms of a set of dimensionless variables and, for this purpose, we choose $\mathrm{H}$ and $\mathrm{U}$ as the characteristic length and velocity and introduce the following 
dimensionless variables of both:

$$
\begin{gathered}
\bar{y}=\frac{y}{H}, \bar{u}=\frac{u}{U}, \bar{\alpha}_{1}=\frac{\alpha_{1}}{(H / U)^{2}}, \quad \bar{\alpha}_{2}=\frac{\alpha_{2}}{(H / U)^{2}}, \bar{x}=\frac{x}{H}, \overline{\hat{P}}=\frac{\hat{P}}{\left(\frac{\mu U}{H}\right)}, \bar{\kappa}^{*}=\frac{\kappa^{*}}{H^{2}}, \\
\theta=\frac{T-T_{2}}{T_{1}-T_{2}}, S=\frac{C-C_{2}}{C_{1}-C_{2}}, \phi=\frac{\widehat{\phi}-\widehat{\phi}_{2}}{\widehat{\phi}_{1}-\widehat{\phi}_{2}} .
\end{gathered}
$$

Thus, the system of our non-linear differential equations (28), (32 - 34) and the boundary conditions (35) can be rewritten in a non-dimensional forms:

$$
\begin{aligned}
& \frac{d}{d \bar{y}}\left[\frac{\left(\frac{d \bar{u}}{d \bar{y}}+\bar{\alpha}_{1}\left(\frac{d \bar{u}}{d \bar{y}}\right)^{3}\right)}{\left(1+\bar{\alpha}_{2}\left(\frac{d \bar{u}}{d \bar{y}}\right)^{2}\right)}\right]-\frac{\partial \bar{P}}{\partial \bar{x}}-F_{S} \bar{u}^{2}-\left(M+\frac{1}{D_{a}}\right) \bar{u}+G_{r} \theta+B_{r} S=0 \\
& \frac{1}{P_{r}} \frac{d^{2} \theta}{d \bar{y}^{2}}+N_{d} \frac{d^{2} S}{d \bar{y}^{2}}+N_{b}\left(\frac{d \theta}{d \bar{y}}\right)\left(\frac{d \phi}{d \bar{y}}\right)+N_{t}\left(\frac{\mathrm{d} \theta}{d \bar{y}}\right)^{2}+\left[\frac{\left(\frac{d \bar{u}}{d \bar{y}}+\bar{\alpha}_{1}\left(\frac{d \bar{u}}{d \bar{y}}\right)^{3}\right)}{\left(1+\bar{\alpha}_{2}\left(\frac{d \bar{u}}{d \bar{y}}\right)^{2}\right)}\right] \frac{d \bar{u}}{d \bar{y}}=0 \\
& \frac{1}{L_{e}} \frac{d^{2} S}{d \bar{y}^{2}}+L_{d} \frac{d^{2} \theta}{d \bar{y}^{2}}-R_{c} S^{m}=0 \\
& N_{b} \frac{d^{2} \phi}{d \bar{y}^{2}}+N_{t} \frac{d^{2} \theta}{d \bar{y}^{2}}=0
\end{aligned}
$$

with the dimensionless boundary conditions:

$$
\begin{aligned}
& \bar{u}=0, \theta=1, S=1, \phi=1 \quad \text { at } \quad \bar{y}=0 \\
& \bar{u}=\bar{U}_{0}, \theta=0, S=0, \phi=0 \quad \text { at } \quad \bar{y}=1
\end{aligned}
$$

Where, the dimensionless parameters are defined by

$$
\begin{gathered}
D_{a}=\frac{\kappa^{*}}{H^{2}}, \quad F_{S}=\frac{\rho c^{*} U H^{2}}{\mu}, \quad M=\frac{\sigma \mu_{e}^{2} H_{0}^{2} H^{2}}{\mu}, G_{r}=\frac{g \alpha_{T} H\left(T_{1}-T_{2}\right)}{U^{2}}, B_{r}=\frac{g \alpha_{C} H\left(C_{1}-C_{2}\right)}{U^{2}}, \\
P_{r}=\frac{\mu U^{2}}{\kappa\left(T_{1}-T_{2}\right)}, \quad N_{d}=\frac{D_{B} \kappa_{T}\left(C_{1}-C_{2}\right)}{C_{S} c_{p} v\left(T_{1}-T_{2}\right)}, N_{b}=\frac{D_{B}\left(\widehat{\phi}_{1}-\widehat{\phi}_{2}\right)}{v}, N_{t}=\frac{D_{T}\left(T_{1}-T_{2}\right)}{T_{1} v}, \\
L_{e}=\frac{v}{D_{B}}, \quad L_{d}=\frac{D_{T} \kappa_{T}\left(T_{1}-T_{2}\right)}{T_{1} v\left(C_{1}-C_{2}\right)}, R_{C}=\frac{H^{2} A\left(C_{1}-C_{2}\right)^{m-1}}{v} .
\end{gathered}
$$

For convenience, we shall drop the bars that identify the dimensionless quantities. The system of non-linear ordinary differential equations (37) - (40) with the boundary conditions (41), will be solved numerically by using the explicit finitedifference method. And, we computed the global error for the solutions of the problem.

\section{NUMERICAL SOLUTION}

The equations (37) - (40) can be written after applied explicit finite difference schemes [6] as:

$$
\begin{aligned}
& \left(\frac{u[i-1]-2 u[i]+u[i+1]}{h^{2}}\right)-\frac{\left[\frac{d \widehat{P}}{d x}+F_{S}(u[i])^{2}+\left(M+\frac{1}{D_{a}}\right) u[i]-G_{r} \theta[i]-B_{r} S[i]\right] \cdot\left[1+\alpha_{2}\left(\frac{u[i]-u[i-1]}{h}\right)^{2}\right]^{2}}{\left[1+3 \alpha_{1}\left(\frac{u[i]-u[i-1]}{h}\right)^{2}-\alpha_{2}\left(\frac{u[i]-u[i-1]}{h}\right)^{2}+\alpha_{1} \alpha_{2}\left(\frac{u[i]-u[i-1]}{h}\right)^{4}\right]}=0 \\
& \left(\frac{\theta[i-1]-2 \theta[i]+\theta[i+1]}{h^{2}}\right)+P_{r} N_{d}\left(\frac{S[i-1]-2 S[i]+S[i+1]}{h^{2}}\right)+P_{r} N_{b}\left(\frac{\theta[i]-\theta[i-1]}{h}\right)\left(\frac{\phi[i]-\phi[i-1]}{h}\right)+P_{r} N_{t}\left(\frac{\theta[i]-\theta[i-1]}{h}\right)^{2}+ \\
& P_{r}\left[\frac{1+\alpha_{1}\left(\frac{u[i]-u[i-1]}{h}\right)^{2}}{1+\alpha_{2}\left(\frac{u[i]-u[i-1]}{h}\right)^{2}}\right]\left(\frac{u[i]-u[i-1]}{h}\right)^{2}=0
\end{aligned}
$$




$$
\begin{aligned}
& \left(\frac{S[i-1]-2 S[i]+S[i+1]}{h^{2}}\right)+L_{e} L_{d}\left(\frac{\theta[i-1]-2 \theta[i]+\theta[i+1]}{h^{2}}\right)-L_{e} R_{c}(S[i])^{m}=0, \\
& \left(\frac{\phi[i-1]-2 \phi[i]+\phi[i+1]}{h^{2}}\right)+N_{t} / N_{b}\left(\frac{\theta[i-1]-2 \theta[i]+\theta[i+1]}{h^{2}}\right)=0 .
\end{aligned}
$$

Where the index $i$ refers to $y$ and the $\Delta y=h=0.04$. According to the boundary conditions (41) we can solved equations (43) - (46) numerically, then a Newtonian iteration method continues until either of goals specified by accuracy goal or precision goal is achieved.

\section{ESTIMATION OF THE GLOBAL ERROR}

We used Zadunaisky technique [19] for calculating the global error, which can be explained in the following steps:

1. We fined the interpolating polynomial of $u, u^{\prime}, \theta, \theta^{\prime}, S, S^{\prime}, \phi, \phi^{\prime}$ from the values of them which came from the explicit finite-difference method. Then we named it $P_{i}(x),(i=1,2,3, \ldots \ldots \ldots, 8)$, and we found the interpolating functions of $u^{\prime \prime}, \theta^{\prime \prime}, S^{\prime \prime}$, and $\phi^{\prime \prime}$, and we named them as:

$$
u^{\prime \prime}=q_{1}(x), \quad \theta^{\prime \prime}=q_{2}(x), \quad S^{\prime \prime}=q_{3}(x), \quad \phi^{\prime \prime}=q_{4}(x),
$$

$q_{1}(x)=\frac{\left[\frac{d \widehat{P}}{d x}+F_{S}\left(P_{1}(x)\right)^{2}+\left(M+\frac{1}{D a}\right) P_{1}(x)-G_{r} P_{3}(x)-B_{r} P_{5}(x)\right] \cdot\left[1+\alpha_{2}\left(P_{2}(x)\right)^{2}\right]^{2}}{\left[1+3 \alpha_{1}\left(P_{2}(x)\right)^{2}-\alpha_{2}\left(P_{2}(x)\right)^{2}+\alpha_{1} \alpha_{2}\left(P_{2}(x)\right)^{4}\right]} \quad$,

$$
q_{2}(x)=\frac{1}{\left(1-P_{r} N_{d} L_{e} L_{d}\right)}\left[-P_{r} N_{d} L_{e} R_{c}\left(P_{5}(x)\right)^{m}-P_{r} N_{b} P_{4}(x) P_{8}(x)-P_{r} N_{t}\left(P_{4}(x)\right)^{2}-P_{r}\left[\frac{1+\alpha_{1}\left(P_{2}(x)\right)^{2}}{1+\alpha_{2}\left(P_{2}(x)\right)^{2}}\right]\left(P_{2}(x)\right)^{2}\right],
$$

$q_{3}(x)=-L_{e} L_{d} q_{2}(x)+L_{e} R_{c}\left(P_{5}(x)\right)^{m} \quad$,

$$
q_{4}(x)=-N_{t} / N_{b} q_{2}(x)
$$

2. We calculate the defect functions $D_{i}(x),(i=1,2,3, \ldots \ldots, 8)$, which can be written as follows:

$$
\begin{array}{cc}
D_{1}(x)=P_{1}^{\prime}(x)-P_{2}(x)=0, & D_{2}(x)=P_{1}^{\prime \prime}(x)-q_{1}(x), \\
D_{3}(x)=P_{3}^{\prime}(x)-P_{4}(x)=0, & D_{4}(x)=P_{3}^{\prime \prime}(x)-q_{2}(x), \\
D_{5}(x)=P_{5}^{\prime}(x)-P_{6}(x)=0, & D_{6}(x)=P_{5}^{\prime \prime}(x)-q_{3}(x), \\
D_{7}(x)=P_{7}^{\prime}(x)-P_{8}(x)=0, & D_{8}(x)=P_{7}^{\prime \prime}(x)-q_{4}(x) .
\end{array}
$$

3. We add the defect functions $D_{i}(x),(i=1,2,3, \ldots \ldots, 8)$ to the original problem.

4. We solved the pseudo-problem (new problem) by the same method which is used for solving the main problem, and we will have the new solutions.

5. We calculate an estimation of the global error from the formulas,

$$
\underline{e}_{n}=\underline{f_{n}}-\underline{f}\left(x_{n}\right)=\underline{f}_{n}-\underline{P}\left(x_{n}\right), \quad(n=0,1,2, \ldots \ldots \ldots, 25)
$$

In this relation, $\underline{f}_{n}$ is the approximate solutions of the new problem (the pseudo-problem) at the point $x_{n}$, and $\underline{f}\left(x_{n}\right)$ is the exact solutions of pseudo-problem at $x_{n}$.

The values of global error for the solutions of the problem which solved by the explicit finite difference method are shown in table (1). The error in table (1) based on using 26 points to find interpolating polynomials $P_{i}(x)$ of degree 25 . In order to achieve the above task, we used the Mathematica package. 
TABLE 1

DIFFERENT VALUES OF THE DIMENSIONLESS PHYSICAL QUANTITIES

\begin{tabular}{|c|c|c|c|c|c|c|c|c|}
\hline \multicolumn{10}{|c|}{ The global error by using finite difference technique } \\
\hline $\mathbf{y}$ & $\mathbf{u}=\mathbf{Y 1}$ & error $(\mathbf{e} 1 \mathbf{n})$ & $\boldsymbol{\theta}=\mathbf{Y 3}$ & $\mathbf{e r r o r}(\mathbf{e} 3 \mathbf{n})$ & $\mathbf{S = Y 5}$ & $\mathbf{e r r o r}\left(\mathbf{e}_{\mathbf{5 n}}\right)$ & $\boldsymbol{\phi}=\mathbf{Y} 7$ & error $(\mathbf{e} 7 \mathbf{n})$ \\
\hline 0 & $0.00 \mathrm{E}+00$ & $0.00 \mathrm{E}+00$ & $1.00 \mathrm{E}+00$ & $0.00 \mathrm{E}+00$ & $1.00 \mathrm{E}+00$ & $0.00 \mathrm{E}+00$ & $1.00 \mathrm{E}+00$ & $0.00 \mathrm{E}+00$ \\
\hline 0.08 & 0.084677 & $2.45938 \mathrm{E}-06$ & 1.0654 & $1.06548 \mathrm{E}-05$ & 0.877711 & $4.73414 \mathrm{E}-06$ & 0.832759 & $9.45491 \mathrm{E}-06$ \\
\hline 0.16 & 0.167804 & $4.11468 \mathrm{E}-06$ & 1.10363 & $1.91716 \mathrm{E}-05$ & 0.765806 & $8.26993 \mathrm{E}-06$ & 0.681824 & $1.69842 \mathrm{E}-05$ \\
\hline 0.24 & 0.249488 & $5.13257 \mathrm{E}-06$ & 1.11623 & $2.58221 \mathrm{E}-05$ & 0.662794 & $1.08326 \mathrm{E}-05$ & 0.546264 & $2.28215 \mathrm{E}-05$ \\
\hline 0.32 & 0.329882 & $5.63641 \mathrm{E}-06$ & 1.10429 & $3.07583 \mathrm{E}-05$ & 0.567513 & $1.25695 \mathrm{E}-05$ & 0.425427 & $2.71033 \mathrm{E}-05$ \\
\hline 0.4 & 0.40918 & $5.70977 \mathrm{E}-06$ & 1.06848 & $3.40436 \mathrm{E}-05$ & 0.479077 & $1.35718 \mathrm{E}-05$ & 0.318913 & $2.98941 \mathrm{E}-05$ \\
\hline 0.48 & 0.487619 & $5.39798 \mathrm{E}-06$ & 1.00906 & $3.56739 \mathrm{E}-05$ & 0.39684 & $1.38901 \mathrm{E}-05$ & 0.226562 & $3.12032 \mathrm{E}-05$ \\
\hline 0.56 & 0.565479 & $4.70768 \mathrm{E}-06$ & 0.925913 & $3.55924 \mathrm{E}-05$ & 0.320364 & $1.3545 \mathrm{E}-05$ & 0.148452 & $3.09962 \mathrm{E}-05$ \\
\hline 0.64 & 0.64308 & $3.60483 \mathrm{E}-06$ & 0.818507 & $3.37017 \mathrm{E}-05$ & 0.249402 & $1.25357 \mathrm{E}-05$ & 0.0848959 & $2.92048 \mathrm{E}-05$ \\
\hline 0.72 & 0.720789 & $2.019 \mathrm{E}-06$ & 0.685922 & $2.98767 \mathrm{E}-05$ & 0.183879 & $1.0847 \mathrm{E}-05$ & 0.0364469 & $2.57363 \mathrm{E}-05$ \\
\hline 0.8 & 0.799017 & $9.48686 \mathrm{E}-08$ & 0.526812 & $2.39868 \mathrm{E}-05$ & 0.123881 & $8.45801 \mathrm{E}-06$ & 0.00391268 & $2.04927 \mathrm{E}-05$ \\
\hline 0.88 & 0.878223 & $2.40736 \mathrm{E}-06$ & 0.339383 & $1.59365 \mathrm{E}-05$ & 0.0696485 & $5.37175 \mathrm{E}-06$ & -0.0116299 & $1.34196 \mathrm{E}-05$ \\
\hline 0.96 & 0.958919 & $2.76915 \mathrm{E}-06$ & 0.121352 & $5.76248 \mathrm{E}-06$ & 0.0215698 & $1.761 \mathrm{E}-06$ & -0.00881092 & $4.69398 \mathrm{E}-06$ \\
\hline $\mathbf{1}$ & $1.00 \mathrm{E}+00$ & $0.00 \mathrm{E}+00$ & $0.00 \mathrm{E}+00$ & $0.00 \mathrm{E}+00$ & $0.00 \mathrm{E}+00$ & $0.00 \mathrm{E}+00$ & $0.00 \mathrm{E}+00$ & $0.00 \mathrm{E}+00$ \\
\hline
\end{tabular}

\section{NUMERICAL RESULTS AND DISCUSSION}

In this paper we generalized the problem of MHD flows to include the non-Newtonian Nano-fluid obeying Oldroyd 6constant model through a porous medium of non-Darcy type with heat and mass transfer. The system of non-linear ordinary differential equations (37) - (40) with the boundary conditions (41) was solved numerically by using an Explicit finite Difference method. The functions $u, \theta, S$, and $\phi$ are obtained and illustrated graphically as shown in figures (a-1 - a-5), (b-1 - b-13), (c-1 - c-13), and (d-1 - d-13) for different values of the parameters of the problem in the case of pure Couette flow $\left(\mathrm{U}_{0}=1, \frac{\mathrm{d} \widehat{\mathrm{P}}=0}{\mathrm{dx}}\right)$, at the values of non-Newtonian parameters $\left(\alpha_{1}=8, \alpha_{2}=2\right)$. And, figures (e-1 - e-4) show the effects of modified pressure gradients on those formula in the case of pure Poiseuille flow $\left(U_{0}=0\right)$, at the values of nonNewtonian parameters $\left(\alpha_{1}=8, \alpha_{2}=2\right)$. And, figures ( $\left.\mathrm{f}-1-\mathrm{f}-4\right)$ show the effects of modified pressure gradients on those formula in the case of generalized Couette flow $\left(U_{0}=1\right)$, at the values of non-Newtonian parameters $\left(\alpha_{1}=8, \alpha_{2}=2\right)$.

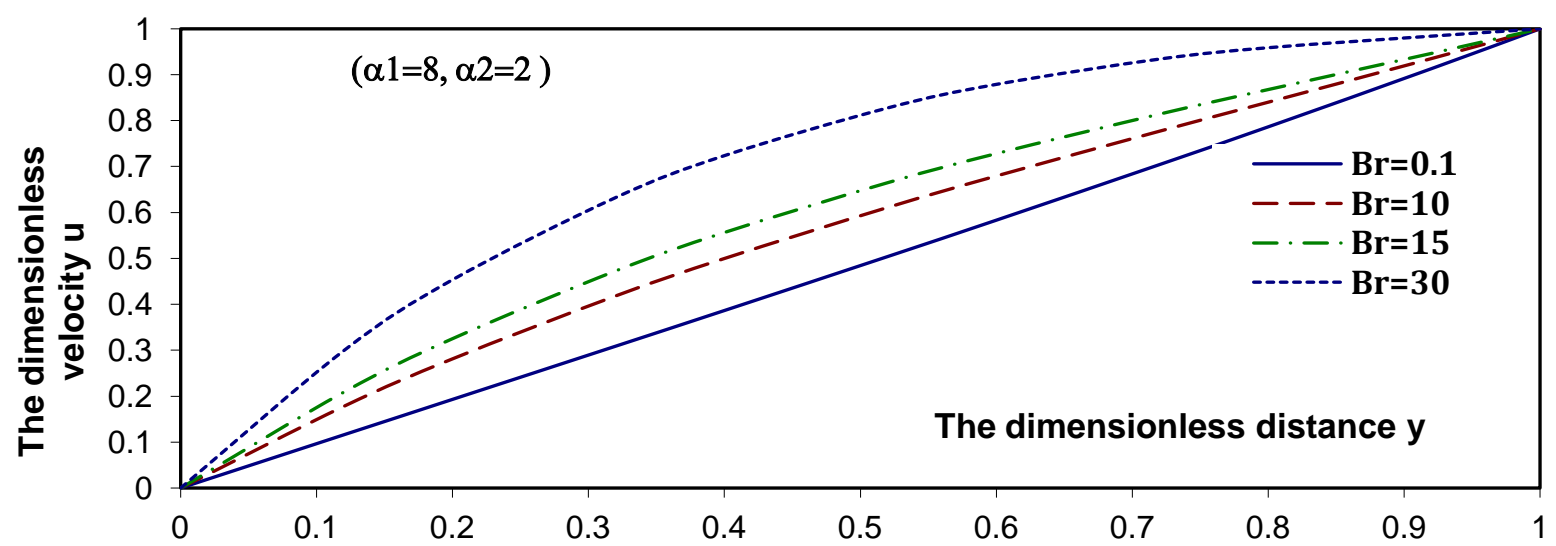

FIG. A-1: Profiles of the velocity $u(y)$ for a pure Couette flow with various values of $B r$ for a system have the particulars $\mathrm{M}=0.1, \mathrm{Da}=1, \mathrm{Fs}=0.5, \mathrm{Gr}=0.1, \mathrm{Pr}=0.7, \mathrm{Nd}=2, \mathrm{Nb}=0.5, \mathrm{Nt}=0.3, \mathrm{Le}=2, \mathrm{Ld}=0.1, \mathrm{Rc}=0.5, \mathrm{~m}=2$. 


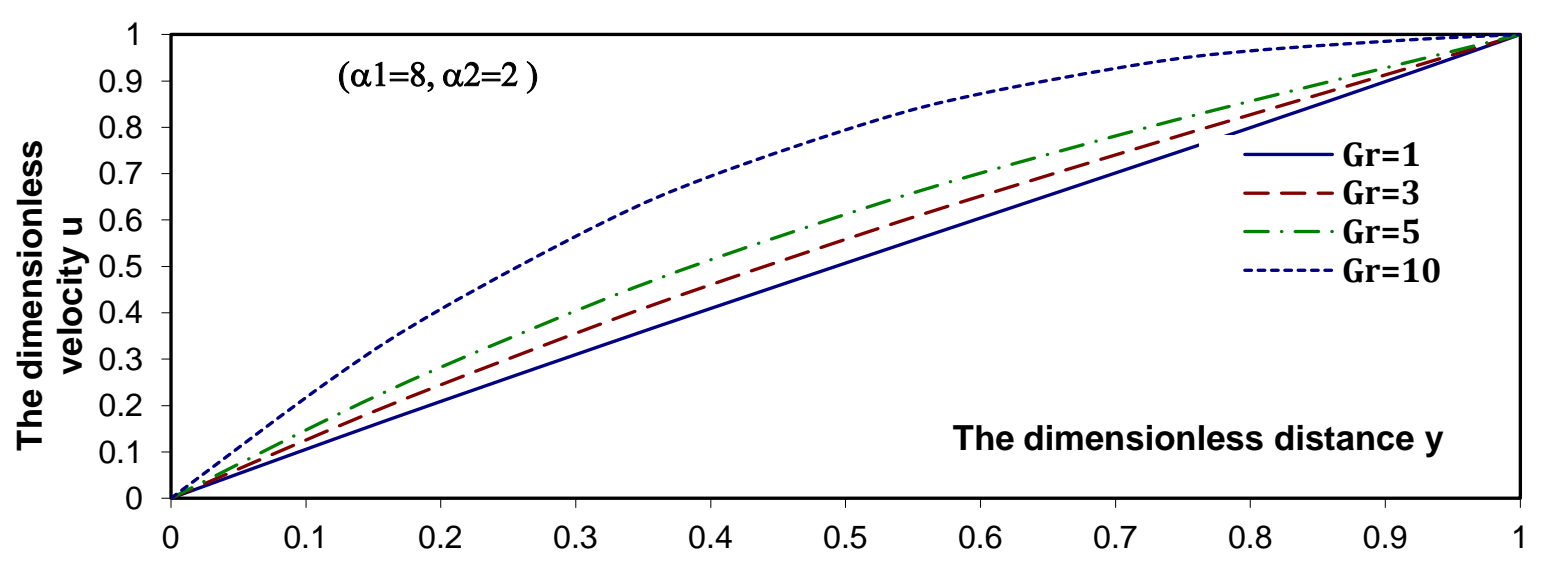

FIG. A-2: Profiles of the velocity $u(y)$ for a pure Couette flow with various values of $\mathrm{Gr}$ for a system have the particulars $\mathrm{M}=0.1, \mathrm{Da}=1, \mathrm{Fs}=0.5, \mathrm{Br}=0.1, \mathrm{Pr}=0.7, \mathrm{Nd}=2, \mathrm{Nb}=0.5, \mathrm{Nt}=0.3, \mathrm{Le}=2, \mathrm{Ld}=0.1, \mathrm{Rc}=0.5, \mathrm{~m}=2$.

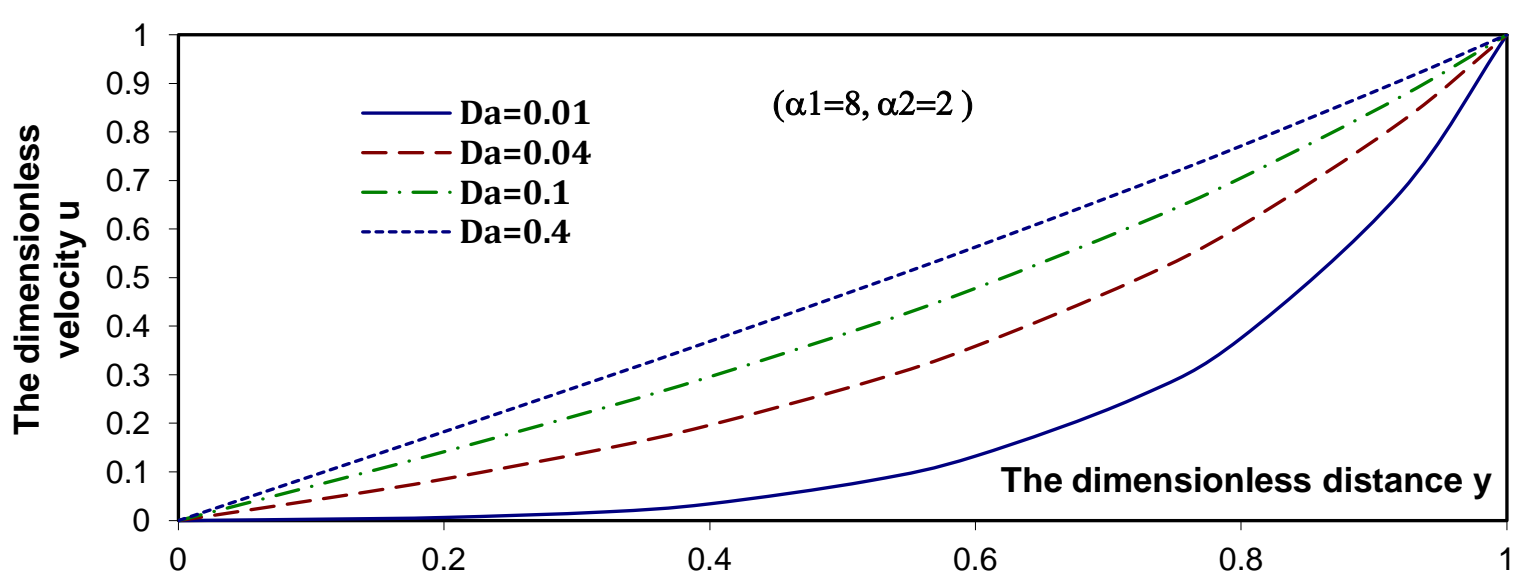

FIG. A-3: Profiles of the velocity $u(y)$ for a pure Couette flow with various values of Da for a system have the particulars $\mathrm{M}=0.1, \mathrm{Fs}=0.5, \mathrm{Nt}=0.3, \mathrm{Gr}=0.1, \mathrm{Br}=0.1, \mathrm{Pr}=0.7, \mathrm{Nd}=2, \mathrm{Nb}=0.5, \mathrm{Nt}=0.3, \mathrm{Le}=2, \mathrm{Ld}=0.1$, $\mathrm{Rc}=0.5, \mathrm{~m}=2$.

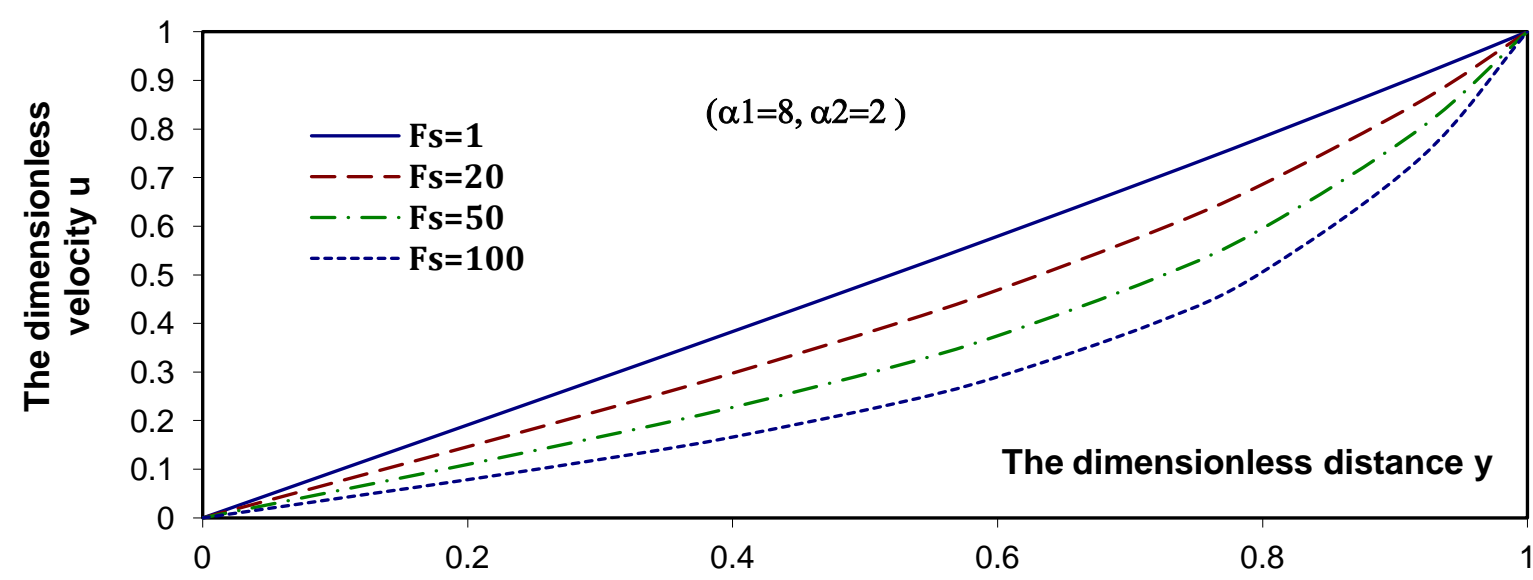

FIG. A-4: Profiles of the velocity $u(y)$ for a pure Couette flow with various values of Fs for a system have the particulars $\mathrm{M}=0.1, \mathrm{Da}=1, \mathrm{Nt}=0.3, \mathrm{Gr}=0.1, \mathrm{Br}=0.1, \mathrm{Pr}=0.7, \mathrm{Nd}=2, \mathrm{Nb}=0.5, \mathrm{Nt}=0.3, \mathrm{Le}=2, \mathrm{Ld}=0.1, \mathrm{Rc}=0.5$, $\mathbf{m}=2$. 


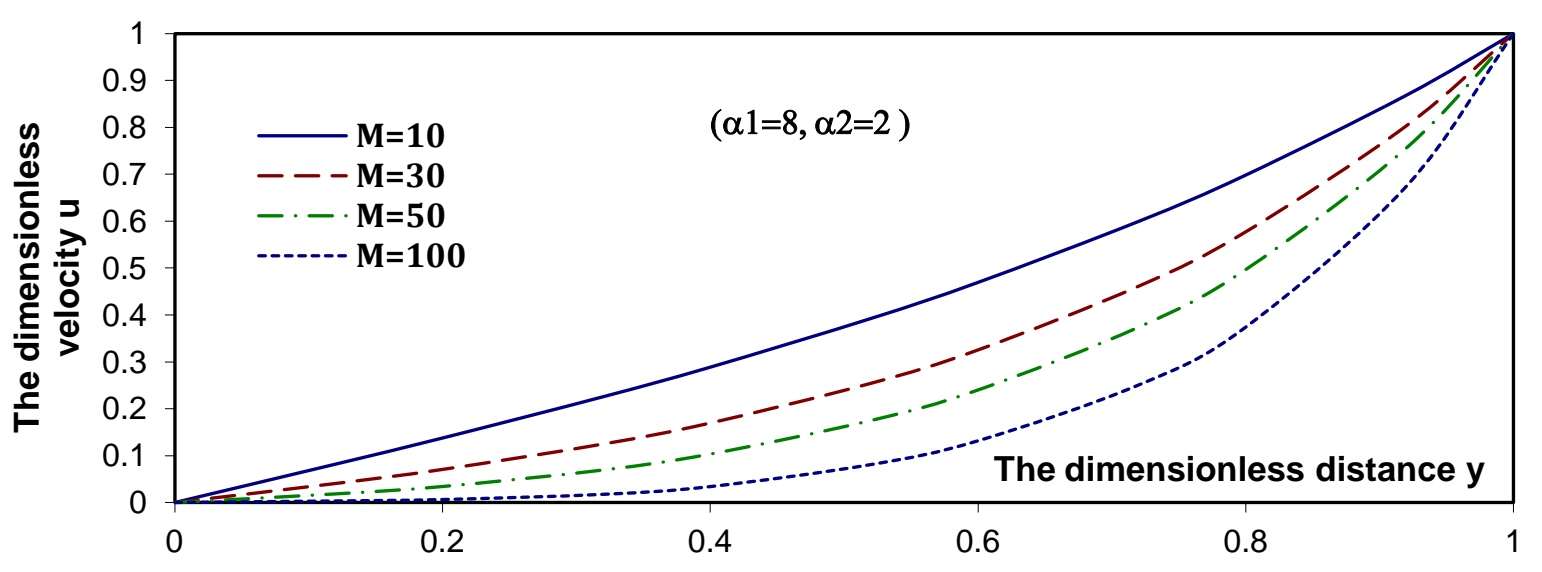

FIG. A-5: Profiles of the velocity $\mathrm{u}(\mathrm{y})$ for a pure Couette flow with various values of $\mathrm{M}$ for a system have the particulars $\mathrm{Da}=1, \mathrm{Fs}=0.5, \mathrm{Nt}=0.3, \mathrm{Gr}=0.1, \mathrm{Br}=0.1, \mathrm{Pr}=0.7, \mathrm{Nd}=2, \mathrm{Nb}=0.5, \mathrm{Nt}=0.3, \mathrm{Le}=2, \mathrm{Ld}=0.1$, $\mathrm{Rc}=0.5, \mathrm{~m}=2$.

\section{1}

\section{Couette flow}

Considering the Couette flow of an Oldroyd 6-constant fluid between two infinite parallel plates, for which the lower plate is fixed and the top plate moves with the velocity $\left(U_{0}=1\right)$, it is assumed that the flow is driven only by the motion of the top plate. The modified pressure gradient in the flow direction vanishes instant $\left(\frac{\mathrm{d} \widehat{\mathrm{P}}}{\mathrm{d}}=0\right)$. Figures (a-1 - a-5), (b-1 - b-13), (c-1 - c13), and (d-1 - d-13) will show the effect of the problem parameters on the problem solutions in this case.

Figures (a-1 - a-5) show the distributions of the velocity profile $u$ at different values of some parameters of the problem. It is clear that, the velocity increases by increasing each of local temperature Grashof number $G_{r}$, local nanoparticle Grashof $B_{r}$, and Darcy number $\mathrm{D}_{\mathrm{a}}$. But, the velocity decreases by increasing each of Forchheimer number $\mathrm{F}_{\mathrm{s}}$, magnetic field parameter $\mathrm{M}$.

Figures (b-1 - b-13) describe the distributions of the temperature profile $\theta$ at different values of some parameters of the problem. It is noted that, as the local nanoparticle Grashof $B_{r}$, local temperature Grashof number $G_{r}$, and Darcy number $D_{a}$ increase, the temperature increases in the region $0 \leq y \leq 0.58$ and, it returns decrease to $0.58 \leq y \leq 1$. The temperature increases by increasing each of Sort number $L_{d}$, Lewis number $L_{e}$, Brownian motion parameter $N_{b}$, Dufour number $N_{d}$, Thermophoresis parameter $\mathrm{N}_{\mathrm{t}}$, Prandtl number $\mathrm{P}_{\mathrm{r}}$, and Chemical reaction parameter $\mathrm{R}_{\mathrm{c}}$. And, as Forchheimer number $\mathrm{F}_{\mathrm{s}}$ increases, the temperature decreases in the region $0 \leq y \leq 0.7$ and, it returns increase to $0.7 \leq y \leq 1$. Also, as magnetic field parameter $\mathrm{M}$ increases, the temperature decreases in the region $0 \leq y \leq 0.5$ and, it returns increase to $0.5 \leq y \leq 1$. Finally, the temperature decreases by increasing Chemical reaction order $\mathrm{m}$.

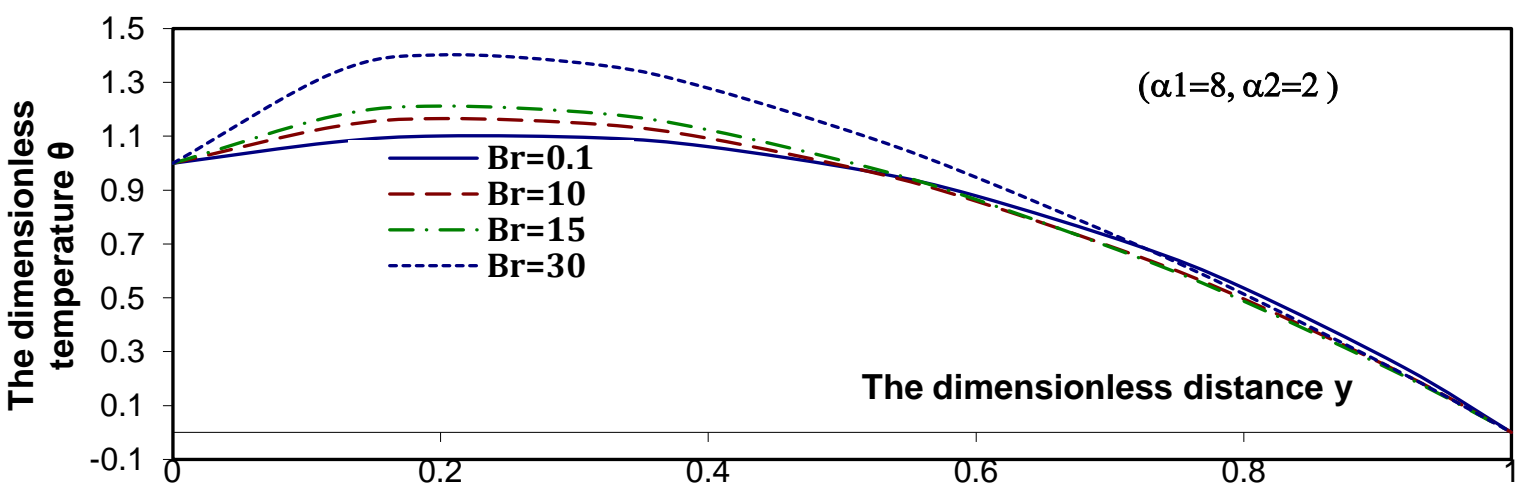

FIG. B-1: Profiles of the temperature $\theta(y)$ for a pure Couette flow with various values of $B r$ for a system have the particulars $\mathrm{M}=0.1, \mathrm{Da}=1, \mathrm{Fs}=0.5, \mathrm{Gr}=0.1, \mathrm{Pr}=0.7, \mathrm{Nd}=2, \mathrm{Nb}=0.5, \mathrm{Nt}=0.3, \mathrm{Le}=2, \mathrm{Ld}=0.1, \mathrm{Rc}=0.5$, $\mathbf{m}=2$. 


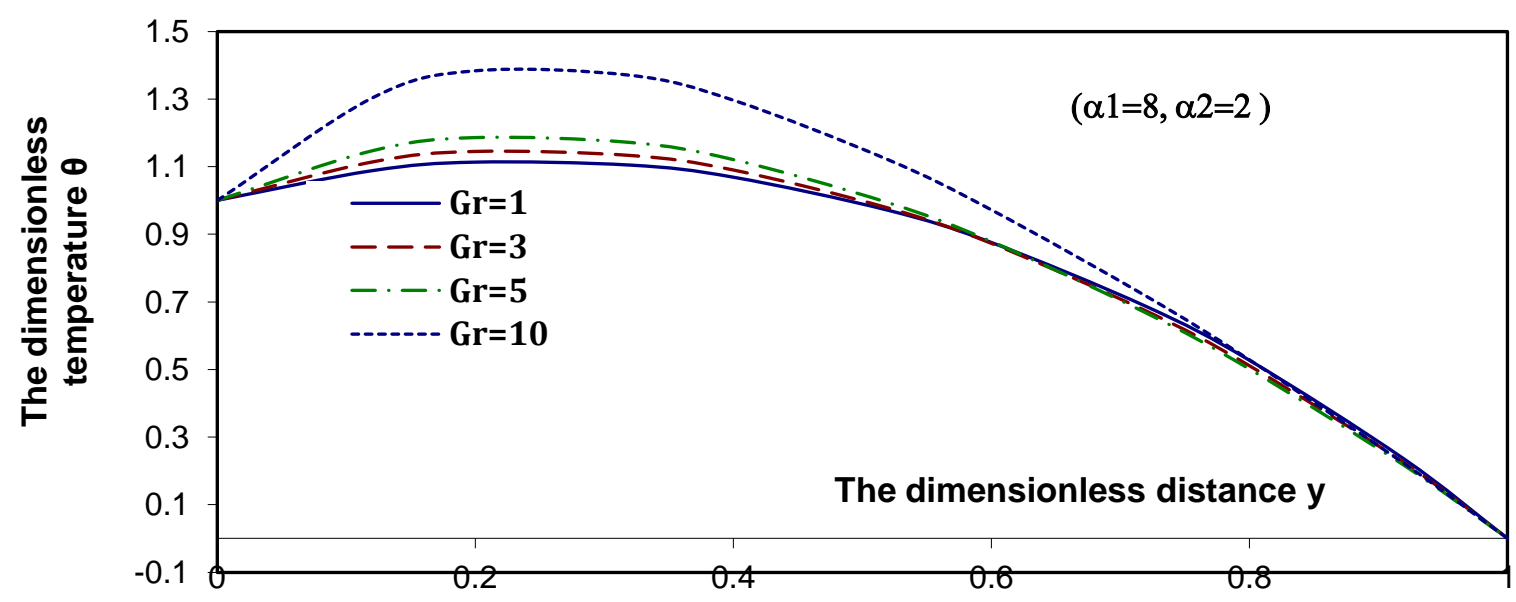

FIG. B-2: Profiles of the temperature $\theta(y)$ for a pure Couette flow with various values of $\mathrm{Gr}$ for a system have the particulars $\mathrm{M}=0.1, \mathrm{Da}=1, \mathrm{Fs}=0.5, \mathrm{Br}=0.1, \mathrm{Pr}=0.7, \mathrm{Nd}=2, \mathrm{Nb}=0.5, \mathrm{Nt}=0.3, \mathrm{Le}=2, \mathrm{Ld}=0.1, \mathrm{Rc}=0.5$, $\mathbf{m}=2$.

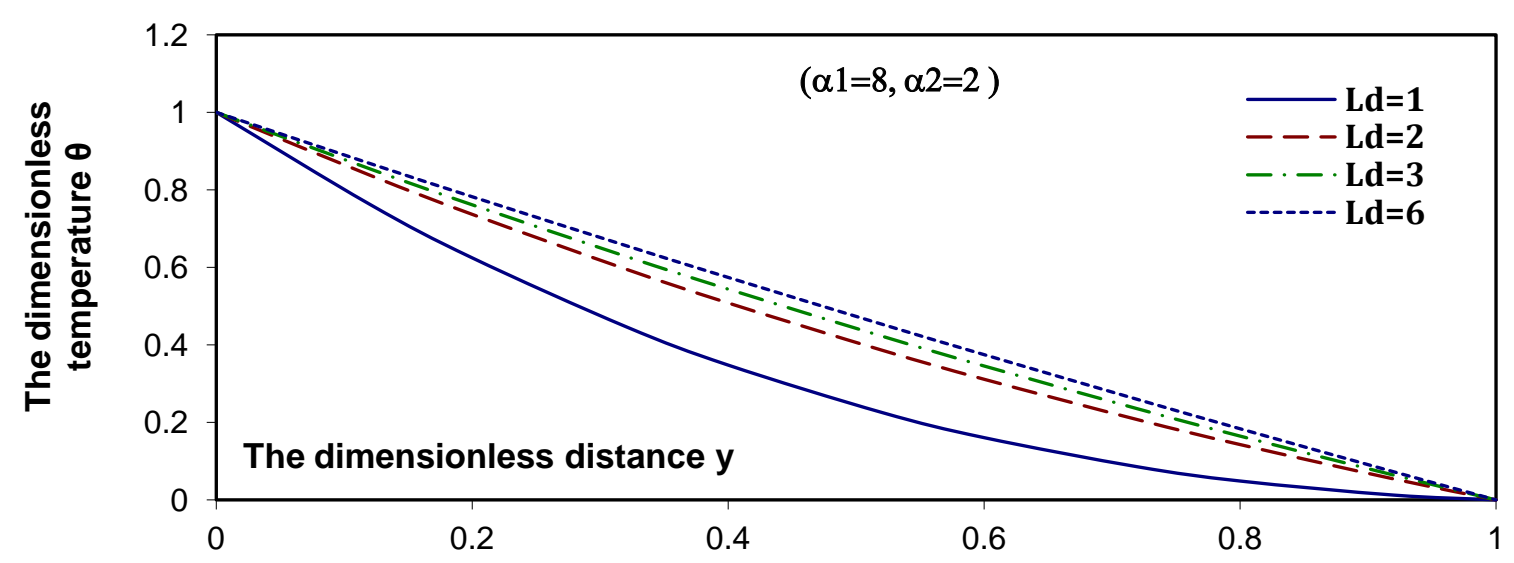

FIG. B-3: Profiles of the temperature $\theta(y)$ for a pure Couette flow with various values $L d$ for a system have the particulars $\mathrm{M}=0.1, \mathrm{Da}=1, \mathrm{Fs}=0.5, \mathrm{Gr}=0.1, \mathrm{Br}=0.1, \mathrm{Pr}=0.7, \mathrm{Nd}=2, \mathrm{Nb}=0.5, \mathrm{Nt}=0.3, \mathrm{Le}=2, \mathrm{Rc}=0.5$, $\mathbf{m}=\mathbf{2}$.

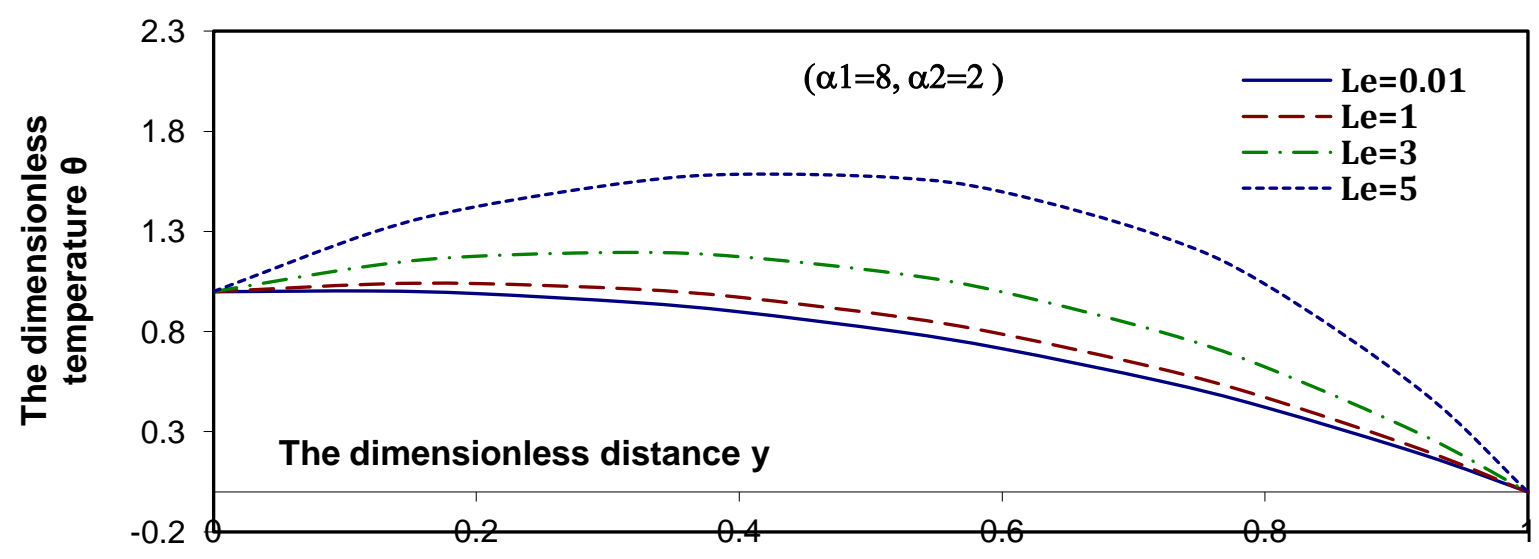

FIG. B-4: Profiles of the temperature $\theta(y)$ for a pure Couette flow with various values of Le for a system have the particulars $\mathrm{M}=0.1, \mathrm{Da}=1, \mathrm{Fs}=0.5, \mathrm{Gr}=0.1, \mathrm{Br}=0.1, \mathrm{Pr}=0.7, \mathrm{Nd}=2, \mathrm{Nb}=0.5, \mathrm{Nt}=0.3, \mathrm{Ld}=0.1, \mathrm{Rc}=0.5$, $\mathbf{m}=\mathbf{2}$. 


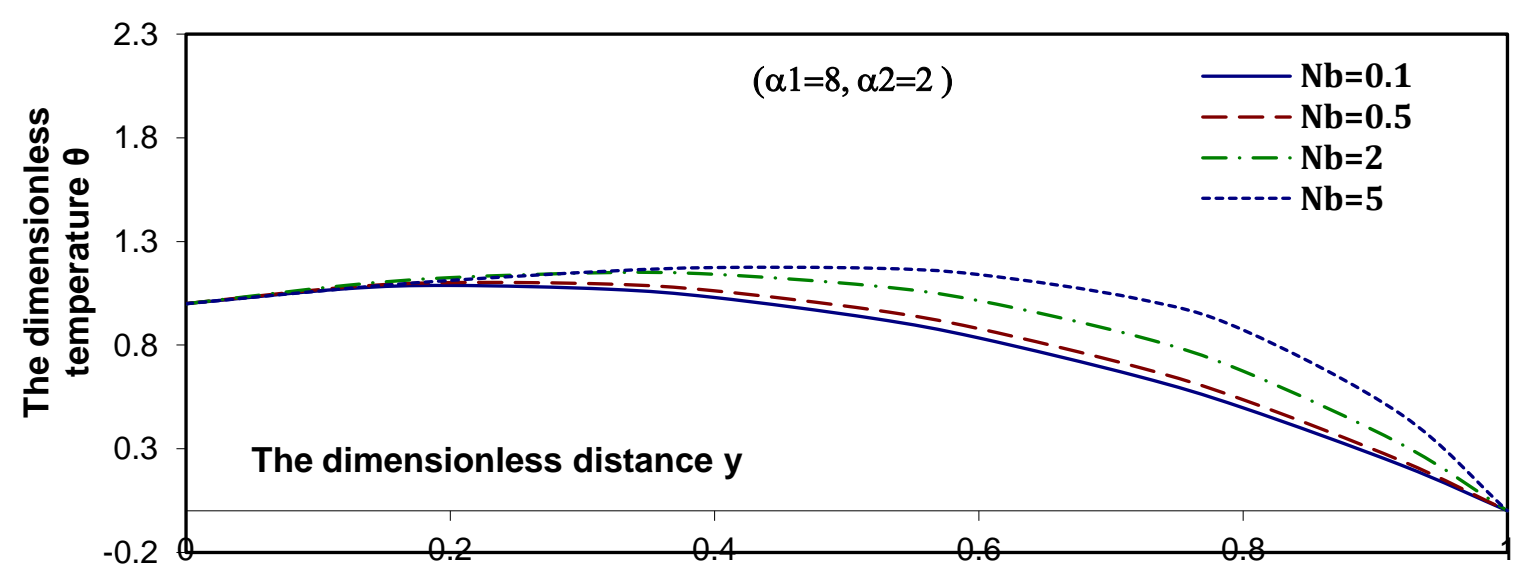

FIG. b-5: Profiles of the temperature $\theta(\mathrm{y})$ for a pure Couette flow with various values of $\mathrm{Nb}$ for a system have the particulars $\mathrm{M}=0.1, \mathrm{Da}=1, \mathrm{Fs}=0.5, \mathrm{Gr}=0.1, \mathrm{Br}=0.1, \mathrm{Pr}=0.7, \mathrm{Nd}=2, \mathrm{Le}=2, \mathrm{Nt}=0.3, \mathrm{Ld}=0.1, \mathrm{Rc}=0.5$, $\mathbf{m}=\mathbf{2}$.

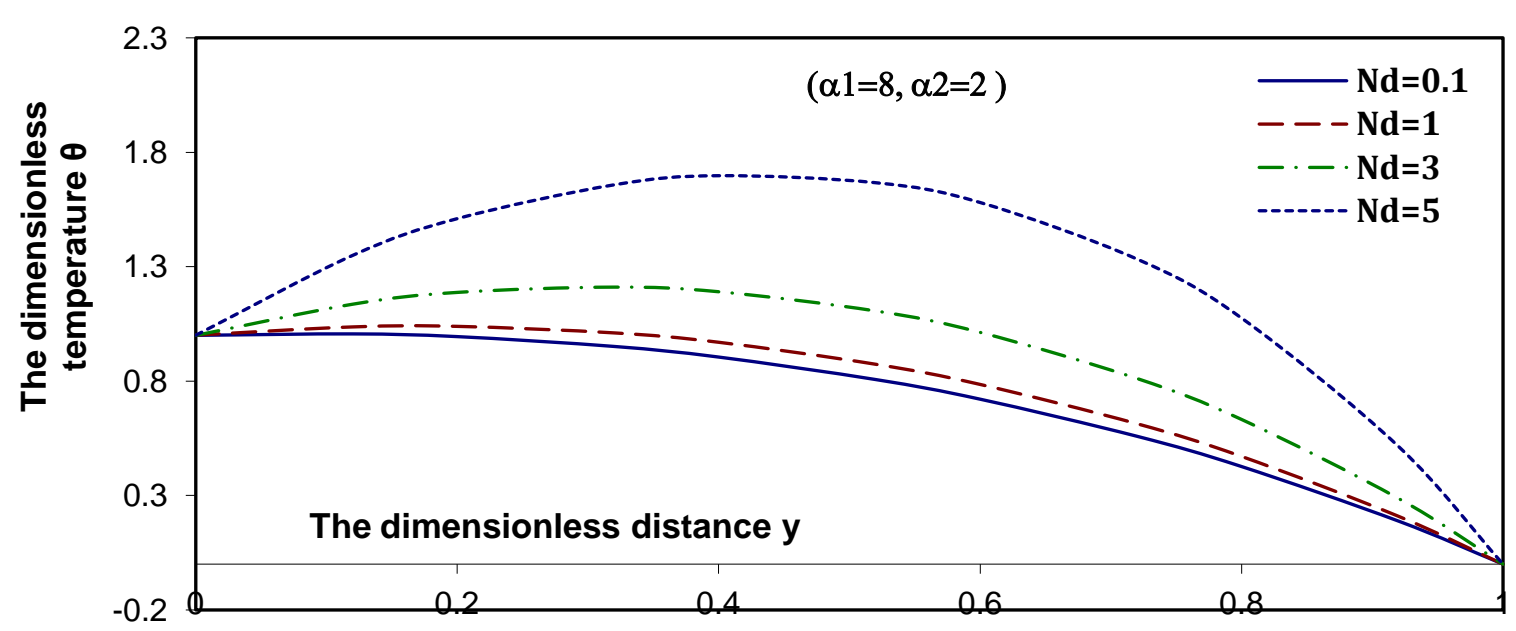

FIG. b-6: Profiles of the temperature $\theta(y)$ for a pure Couette flow with various values of $\mathrm{Nd}$ for a system have the particulars $\mathrm{M}=0.1, \mathrm{Da}=1, \mathrm{Fs}=0.5, \mathrm{Gr}=0.1, \mathrm{Br}=0.1, \mathrm{Pr}=0.7, \mathrm{Nb}=0.5, \mathrm{Le}=2, \mathrm{Nt}=0.3, \mathrm{Ld}=0.1, \mathrm{Rc}=0.5$, $\mathbf{m}=2$.

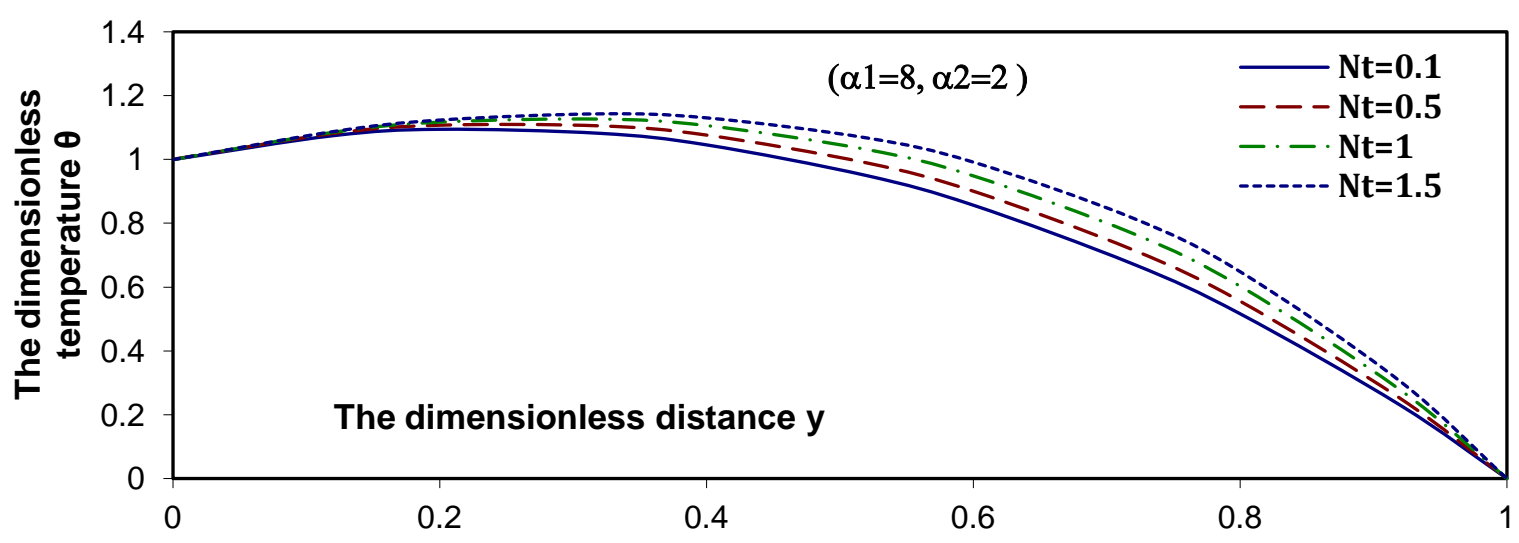

FIG. b-7: Profiles of the temperature $\theta(y)$ for a pure Couette flow with various values of $\mathrm{Nt}$ for a system have the particulars $\mathrm{M}=0.1, \mathrm{Da}=1, \mathrm{Fs}=0.5, \mathrm{Gr}=0.1, \mathrm{Br}=0.1, \mathrm{Pr}=0.7, \mathrm{Nb}=0.5, \mathrm{Le}=2, \mathrm{Nd}=2, \mathrm{Ld}=0.1, \mathrm{Rc}=0.5$, $\mathbf{m}=2$. 


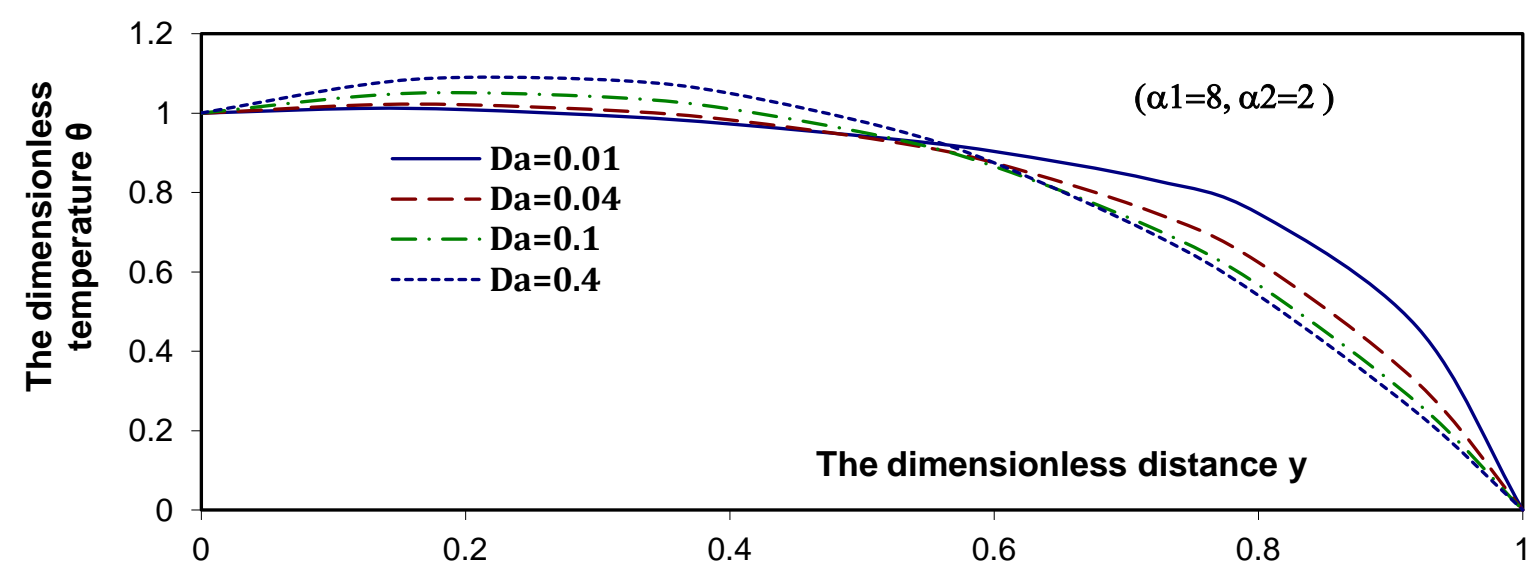

FIG. b-8: Profiles of the temperature $\theta(y)$ for a pure Couette flow with various values of $D a$ for a system have the particulars $\mathrm{M}=0.1, \mathrm{Fs}=0.5=1, \mathrm{Nt}=0.3, \mathrm{Gr}=0.1, \mathrm{Br}=0.1, \mathrm{Pr}=0.7, \mathrm{Nd}=2, \mathrm{Nb}=0.5, \mathrm{Nt}=0.3, \mathrm{Le}=2$, $\mathrm{Ld}=0.1, \mathrm{Rc}=0.5, \mathrm{~m}=2$.

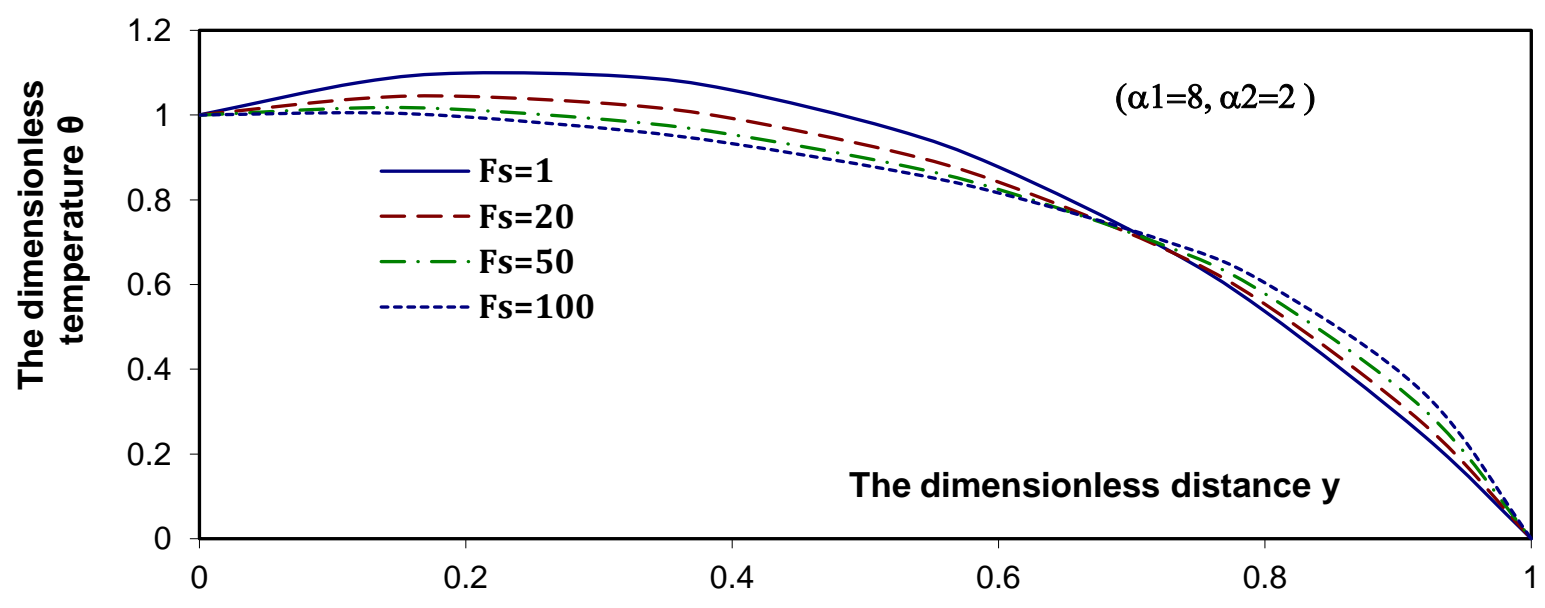

FIG. b-9: Profiles of the temperature $\theta(y)$ for a pure Couette flow with various values of Fs for a system have the particulars $\mathrm{M}=0.1, \mathrm{Da}=1, \mathrm{Nt}=0.3, \mathrm{Gr}=0.1, \mathrm{Br}=0.1, \mathrm{Pr}=0.7, \mathrm{Nd}=2, \mathrm{Nb}=0.5, \mathrm{Nt}=0.3, \mathrm{Le}=2, \mathrm{Ld}=0.1$, $\mathrm{Rc}=\mathbf{0 . 5}, \mathrm{m}=\mathbf{2}$.

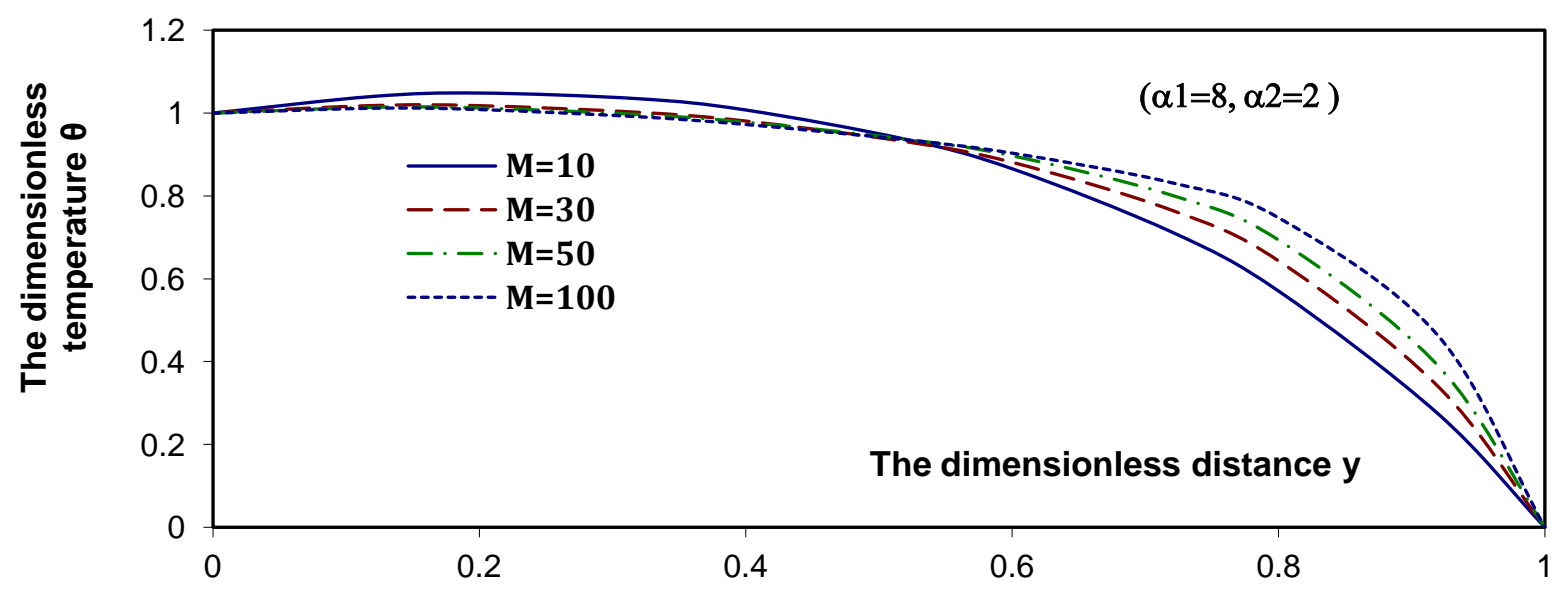

FIG. b-10: Profiles of the temperature $\theta(y)$ for a pure Couette flow with various values of $M$ for a system have the particulars $\mathrm{Da}=1, \mathrm{Fs}=0.5=1, \mathrm{Nt}=0.3, \mathrm{Gr}=0.1, \mathrm{Br}=0.1, \mathrm{Pr}=0.7, \mathrm{Nd}=2, \mathrm{Nb}=0.5, \mathrm{Nt}=0.3, \mathrm{Le}=2, \mathrm{Ld}=0.1$, $\mathrm{Rc}=0.5, \mathrm{~m}=2$. 


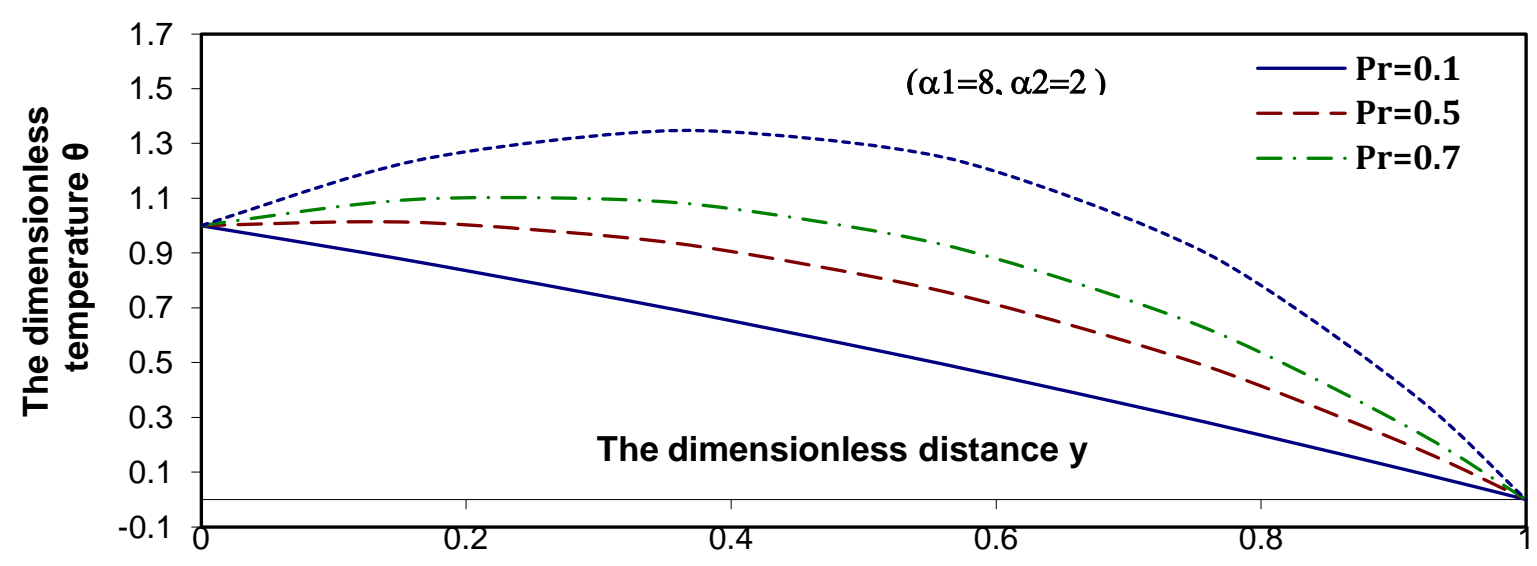

FIG. b-11: Profiles of the temperature $\theta(y)$ for a pure Couette flow with various values of Pr for a system have the particulars $\mathrm{Da}=1, \mathrm{Fs}=0.5=1, \mathrm{Nt}=0.3, \mathrm{Gr}=0.1, \mathrm{Br}=0.1, \mathrm{M}=0.1, \mathrm{Nd}=2, \mathrm{Nb}=0.5, \mathrm{Nt}=0.3, \mathrm{Le}=2, \mathrm{Ld}=0.1$, $\mathrm{Rc}=0.5, \mathrm{~m}=2$.

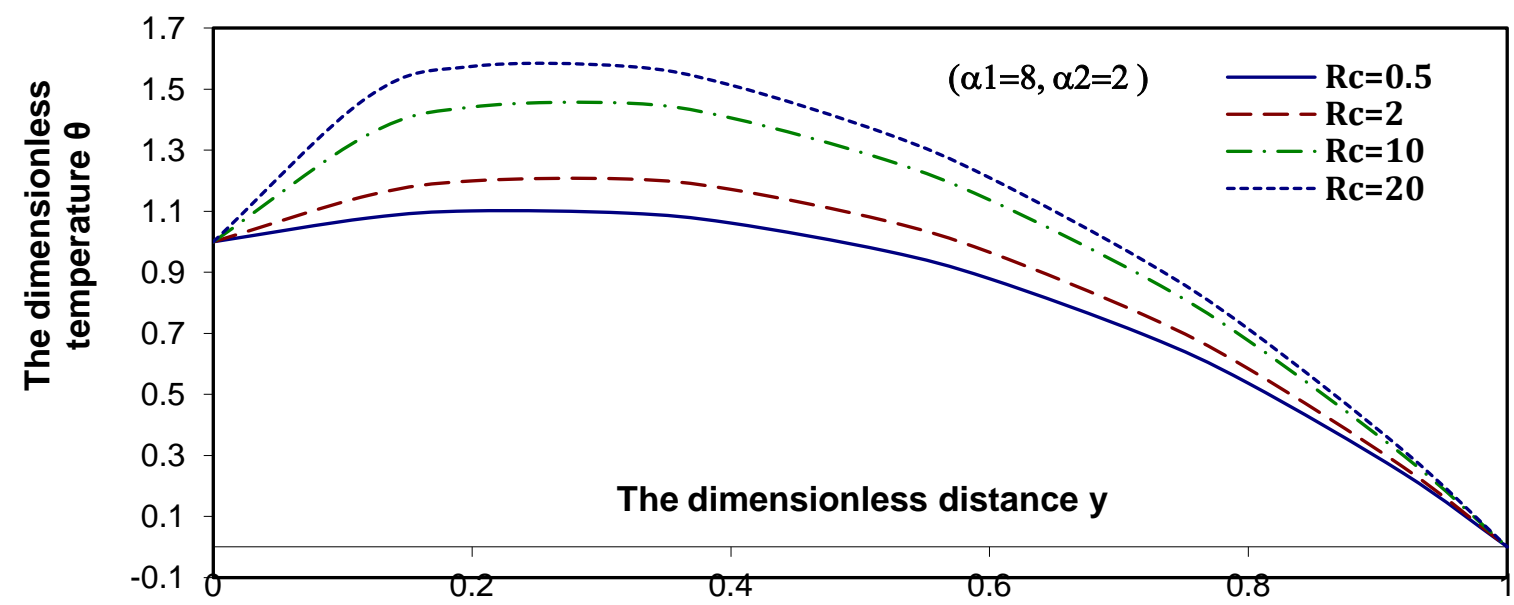

FIG. b-12: Profiles of the temperature $\theta(y)$ for a pure Couette flow with various values of Rc for a system have the particulars $\operatorname{Pr}=0.7, \mathrm{Da}=1, \mathrm{Fs}=0.5=1, \mathrm{Nt}=0.3, \mathrm{Gr}=0.1, \mathrm{Br}=0.1, \mathrm{M}=0.1, \mathrm{Nd}=2, \mathrm{Nb}=0.5, \mathrm{Nt}=0.3, \mathrm{Le}=2$, $\mathrm{Ld}=\mathbf{0 . 1}, \mathrm{m}=\mathbf{2}$.

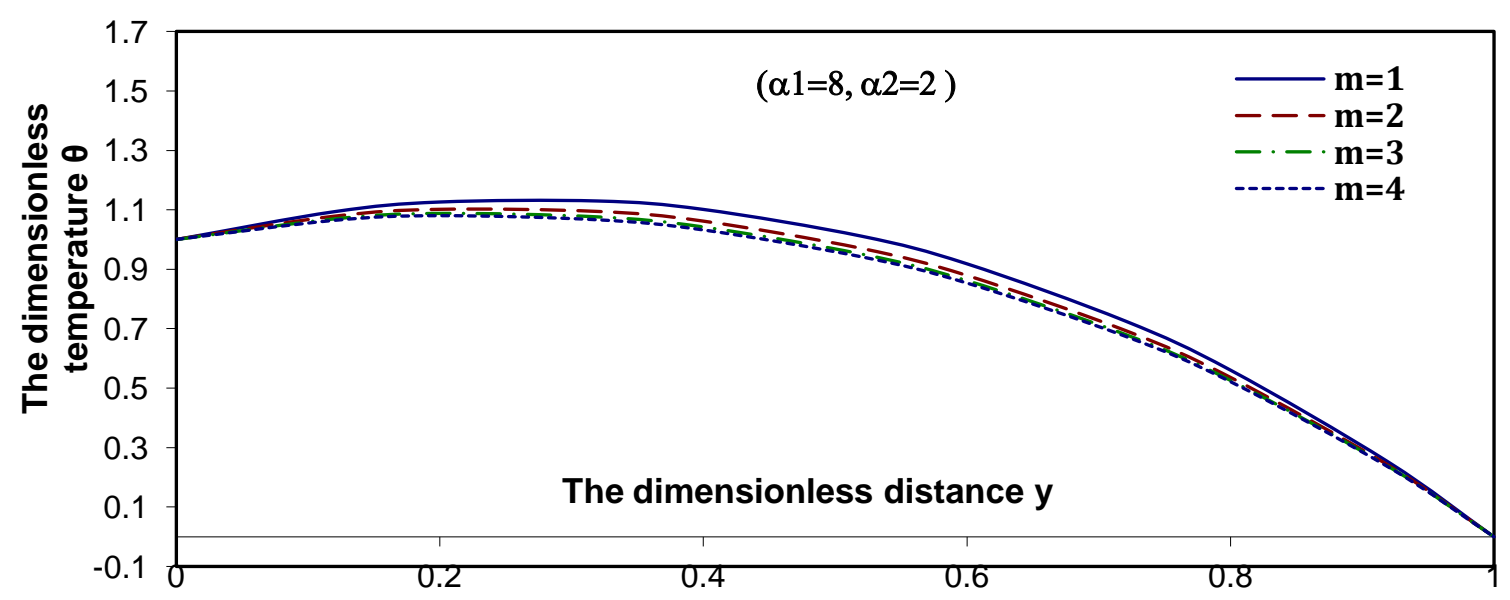

FIG. b-13: Profiles of the temperature $\theta(y)$ for a pure Couette flow with various values of $\mathbf{m}$ for a system have the particulars $\mathrm{Pr}=0.7, \mathrm{Da}=1, \mathrm{Fs}=0.5=1, \mathrm{Nt}=0.3, \mathrm{Gr}=0.1, \mathrm{Br}=0.1, \mathrm{M}=0.1, \mathrm{Nd}=2, \mathrm{Nb}=0.5, \mathrm{Nt}=0.3, \mathrm{Le}=2$, $L d=0.1, R c=0.5$. 
Figures (c-1 - c-13) illustrate the distributions of the concentration profile $S$ at different values of some parameters of the problem. It is seen that, the concentration decreases by increasing each of local nanoparticle Grashof $B_{r}$, local temperature Grashof number $G_{r}$, Sort number $L_{d}$, Lewis number $L_{e}$, Brownian motion parameter $N_{b}$, Dufour number $N_{d}$, Thermophoresis parameter $\mathrm{N}_{\mathrm{t}}$, Prandtl number $\mathrm{P}_{\mathrm{r}}$, and Chemical reaction parameter $\mathrm{R}_{\mathrm{c}}$. And, as Darcy number $\mathrm{D}_{\mathrm{a}}$ increases, the concentration decreases in the region $0 \leq y \leq 0.6$ and, it returns increase to $0.6 \leq y \leq 1$. But, as Forchheimer number $\mathrm{F}_{\mathrm{s}}$ increases, the concentration increases in the region $0 \leq y \leq 0.7$ and, it returns decrease to $0.7 \leq y \leq 1$. The effect of magnetic field parameter $\mathrm{M}$ on the concentration disappears in the region $0 \leq y \leq 0.6$, the concentration returns decrease to $0.6 \leq y \leq 1$ at large values of M. Finally, the concentration increases by increasing Chemical reaction order $\mathrm{m}$.

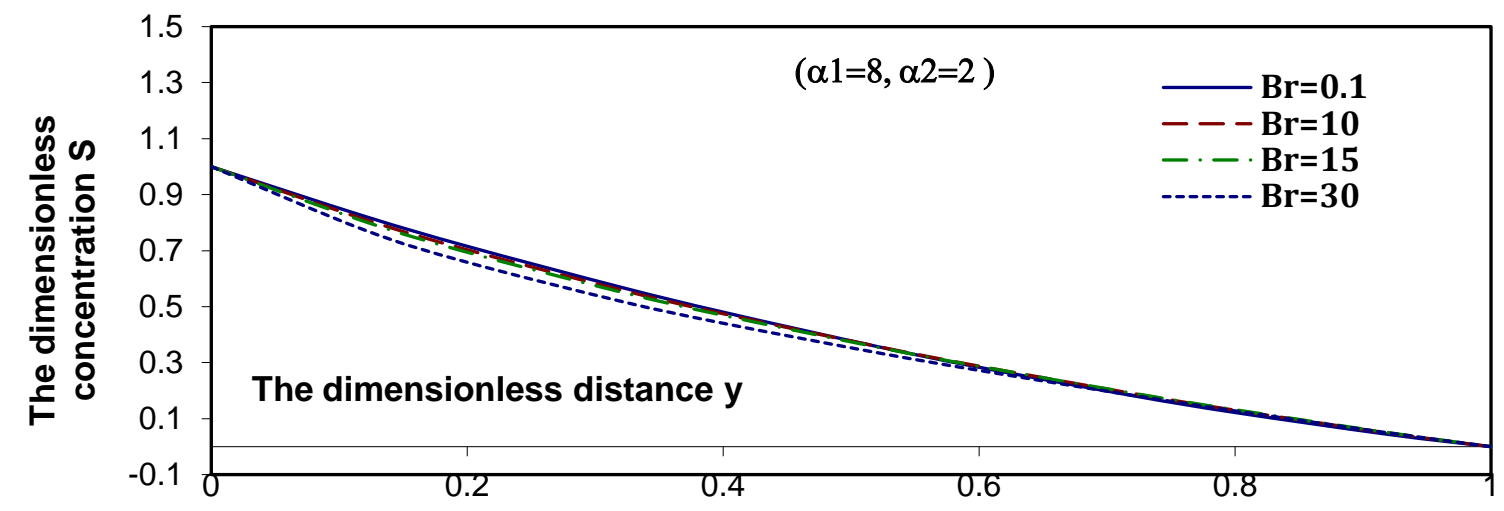

FIG. c-1: Profiles of the concentration $S(y)$ for a pure Couette flow with various values of $B r$ for a system have the particulars M=0.1, Da=1, Fs=0.5, Gr=0.1, $\mathrm{Pr}=0.7, \mathrm{Nd}=2, \mathrm{Nb}=0.5, \mathrm{Nt}=0.3, \mathrm{Le}=2, \mathrm{Ld}=0.1, \mathrm{Rc}=0.5, \mathrm{~m}=2$.

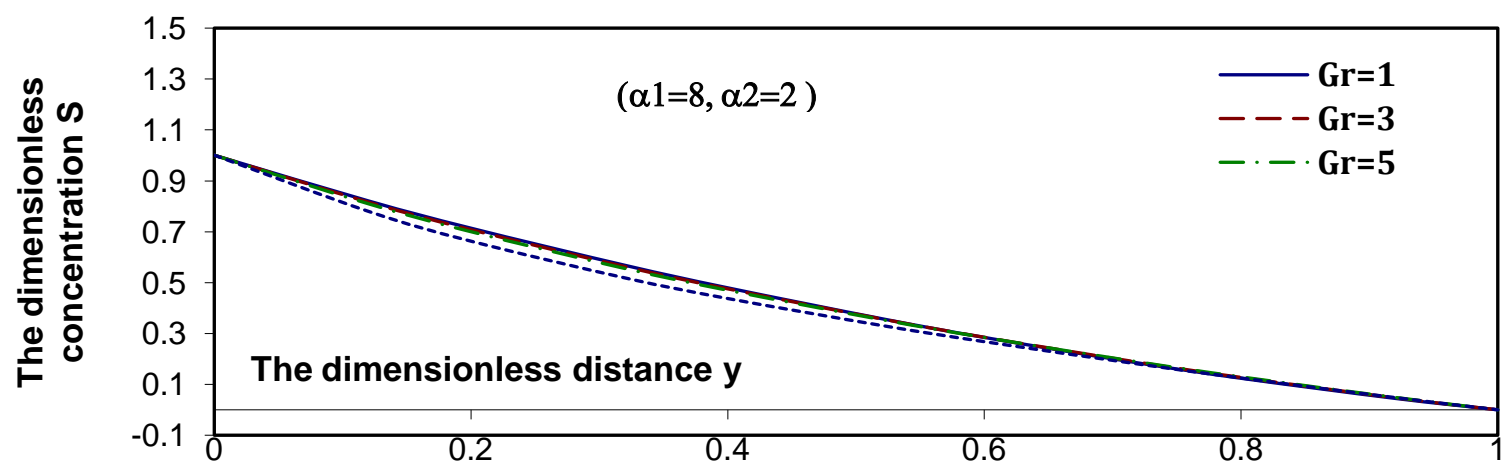

FIG. c-2: Profiles of the concentration $\mathrm{S}(\mathrm{y})$ for a pure Couette flow with various values of $\mathrm{Gr}$ for a system have the particulars $\mathrm{M}=0.1, \mathrm{Da}=1, \mathrm{Fs}=0.5, \mathrm{Br}=0.1, \mathrm{Pr}=0.7, \mathrm{Nd}=2, \mathrm{Nb}=0.5, \mathrm{Nt}=0.3, \mathrm{Le}=2, \mathrm{Ld}=0.1, \mathrm{Rc}=0.5$, $\mathbf{m}=2$.

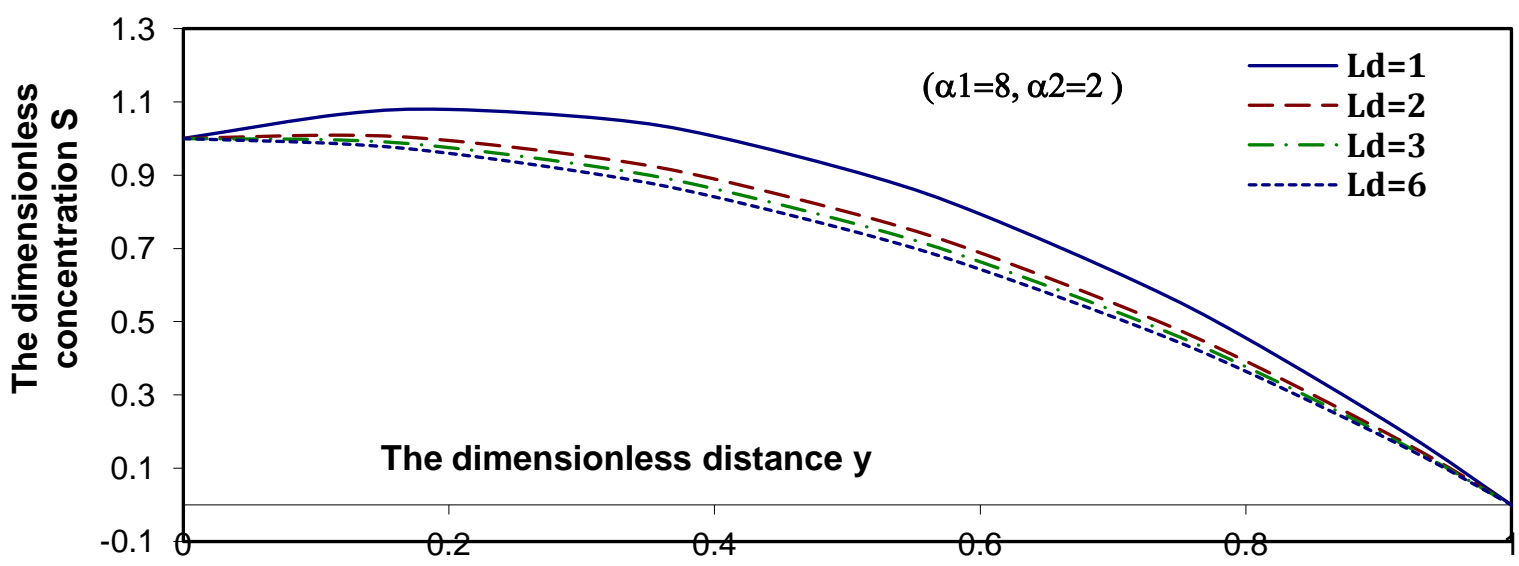

FIG. c-3: Profiles of the concentration $S(y)$ for a pure Couette flow with various values of Ld for a system have the particulars $\mathrm{M}=0.1, \mathrm{Da}=1, \mathrm{Fs}=0.5, \mathrm{Gr}=0.1, \mathrm{Br}=0.1, \mathrm{Pr}=0.7, \mathrm{Nd}=2, \mathrm{Nb}=0.5, \mathrm{Nt}=0.3, \mathrm{Le}=2, \mathrm{Rc}=0.5, \mathrm{~m}=2$. 


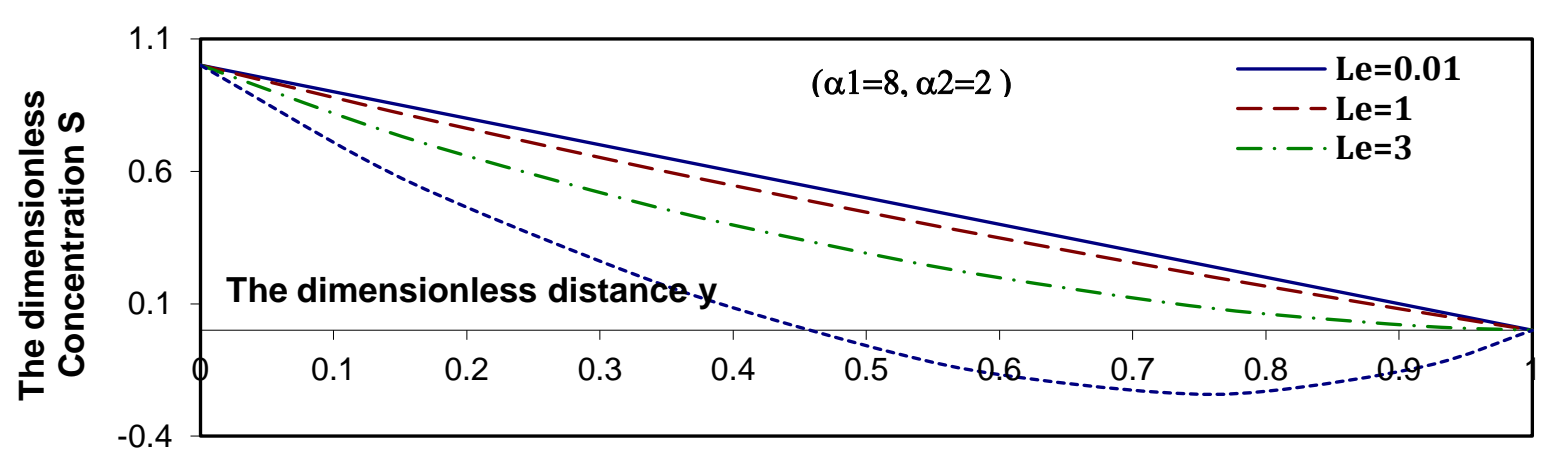

FIG. c-4: Profiles of the Concentration S(y) for a pure Couette flow with various values of Le for a system have the particulars M=0.1, Da=1, Fs=0.5, Gr=0.1, Br=0.1, Pr=0.7, Nd=2, Nb=0.5, Nt=0.3, Ld=0.1, Rc=0.5, m=2.

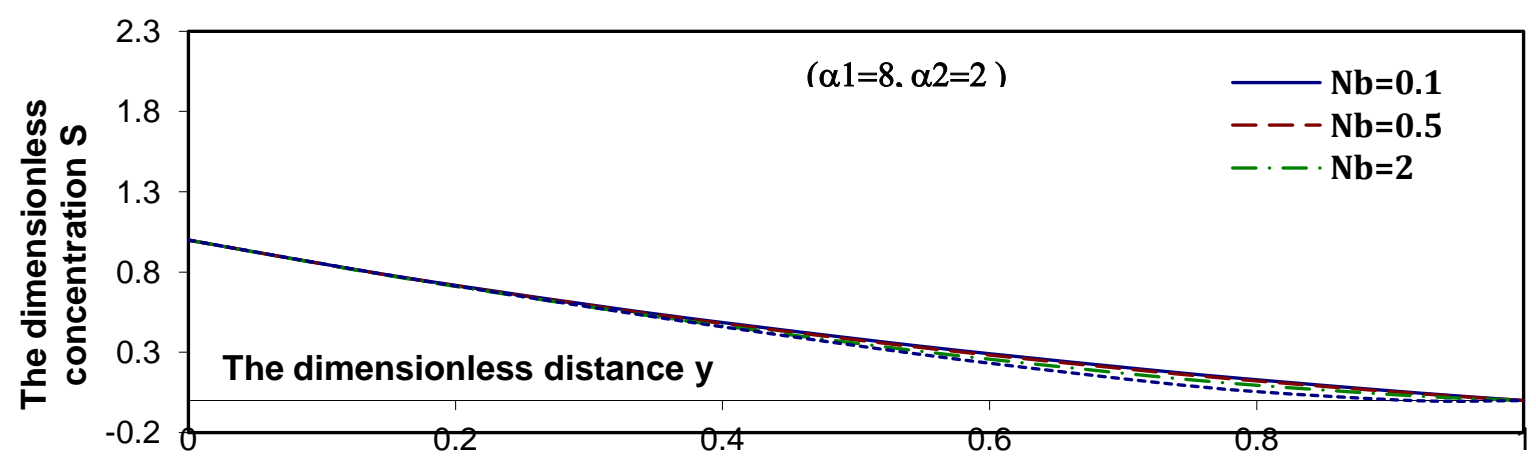

FIG. c-5: Profiles of the concentration $\mathrm{S}(\mathrm{y})$ for a pure Couette flow with various values of $\mathrm{Nb}$ for a system have the particulars M=0.1, Da=1, Fs=0.5, Gr=0.1, Br=0.1, Pr=0.7, Nd=2, Le=2, Nt=0.3, Ld=0.1, Rc=0.5, m=2.

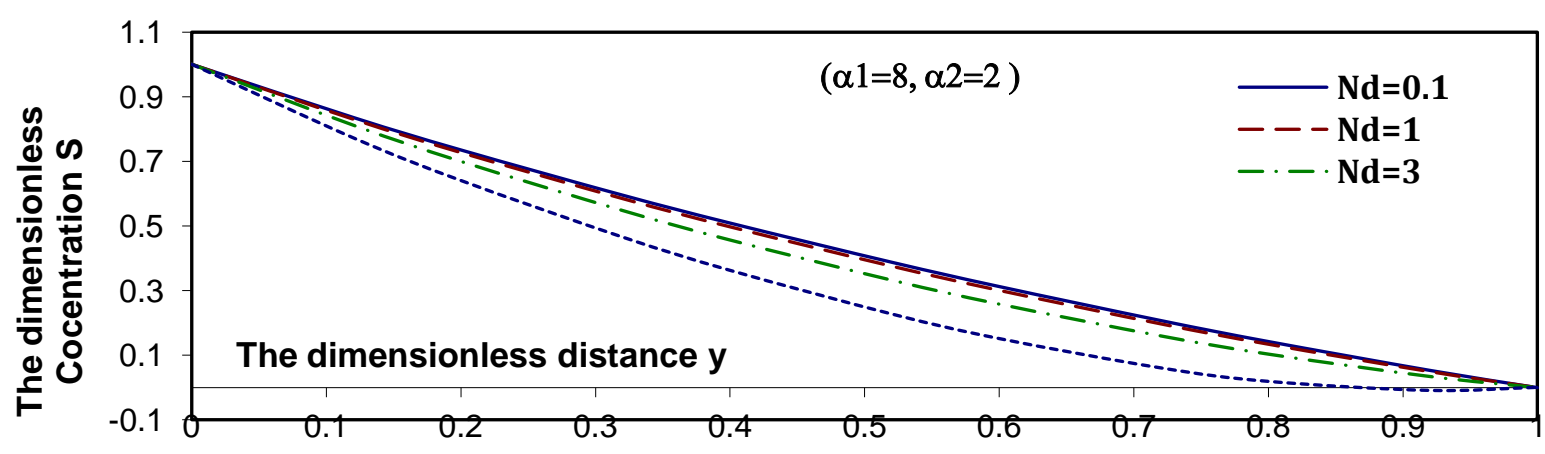

FIG. c-6: Profiles of the Concentration $\mathrm{S}(\mathrm{y})$ for a pure Couette flow with various values of $\mathrm{Nd}$ for a system have the particulars M=0.1, Da=1, Fs=0.5, Gr=0.1, Br=0.1, Pr=0.7, Nb=0.5, Le=2, Nt=0.3, Ld=0.1, Rc=0.5, m=2.

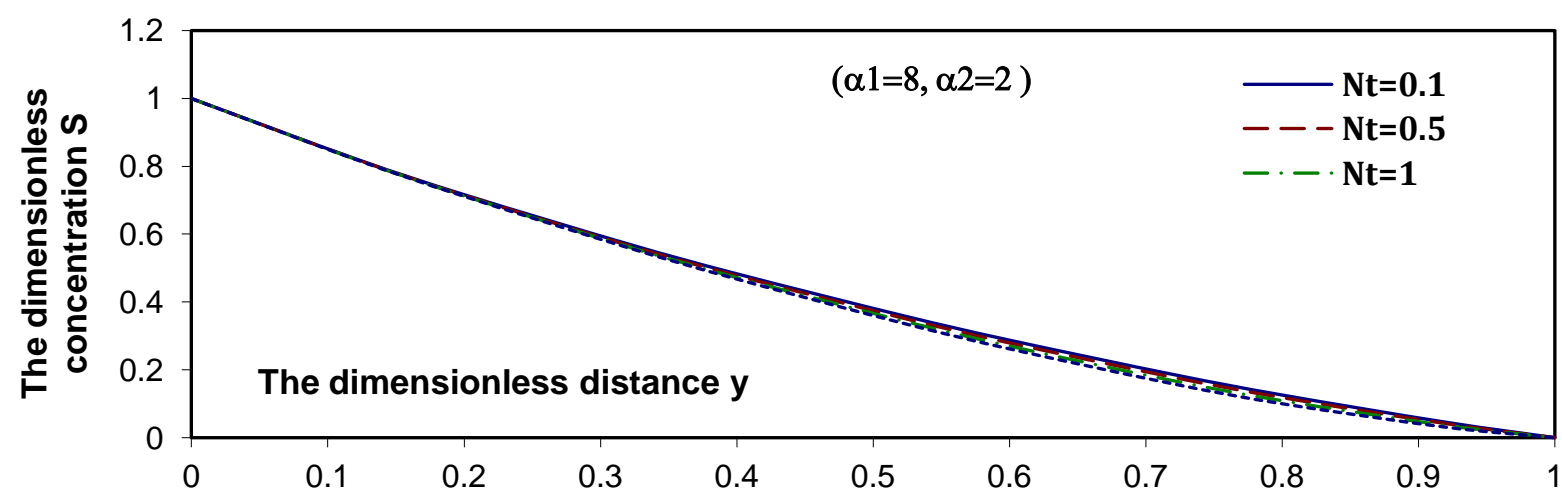

FIG. c-7: Profiles of the Concentration S(y) for a pure Couette flow with various values of $\mathrm{Nt}$ for a system have the particulars M=0.1, Da=1, Fs=0.5, Gr=0.1, Br=0.1, $\mathrm{Pr}=0.7, \mathrm{Nb}=0.5, \mathrm{Le}=2, \mathrm{Nd}=2, \mathrm{Ld}=0.1, \mathrm{Rc}=0.5, \mathrm{~m}=2$. 


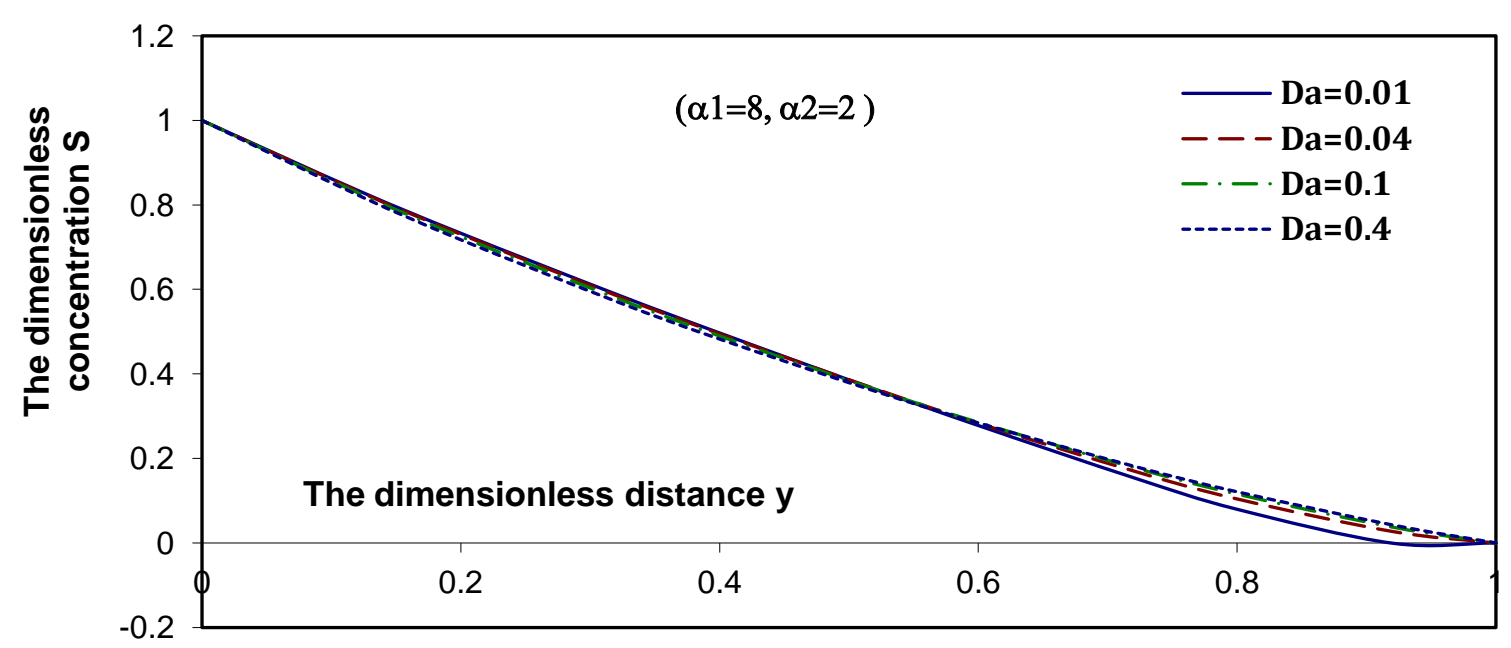

FIG. c-8: Profiles of the concentration $S(y)$ for a pure Couette flow with various values of Da for a system have the particulars $\mathrm{M}=0.1, \mathrm{Fs}=1, \mathrm{Nt}=0.3, \mathrm{Gr}=0.1, \mathrm{Br}=0.1, \mathrm{Pr}=0.7, \mathrm{Nd}=2, \mathrm{Nb}=0.5, \mathrm{Nt}=0.3, \mathrm{Le}=2, \mathrm{Ld}=0.1, \mathrm{Rc}=0.5, \mathrm{~m}=2$.

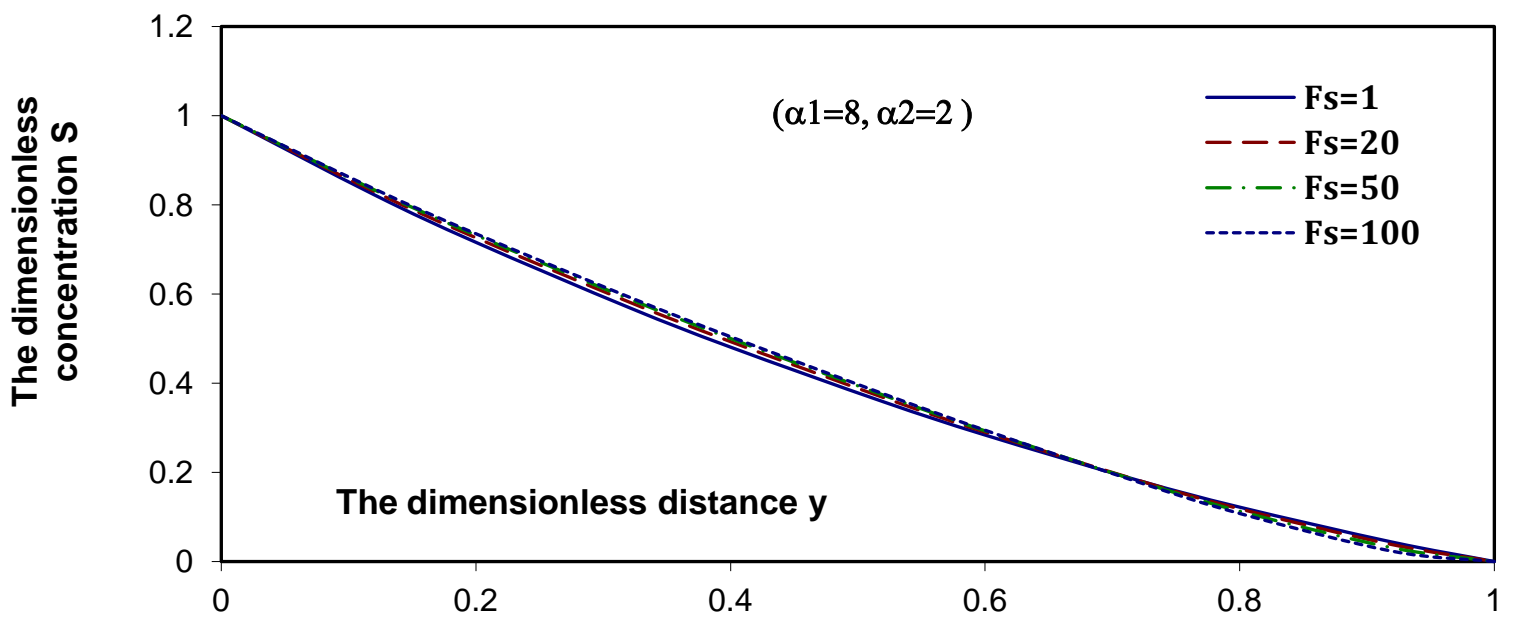

FIG. c-9: Profiles of the concentration S(y) for a pure Couette flow with various values of Fs for a system have the particulars $\mathrm{M}=0.1, \mathrm{Da}=1, \mathrm{Nt}=0.3, \mathrm{Gr}=0.1, \mathrm{Br}=0.1, \mathrm{Pr}=0.7, \mathrm{Nd}=2, \mathrm{Nb}=0.5, \mathrm{Nt}=0.3, \mathrm{Le}=2, \mathrm{Ld}=0.1, \mathrm{Rc}=0.5, \mathrm{~m}=2$.

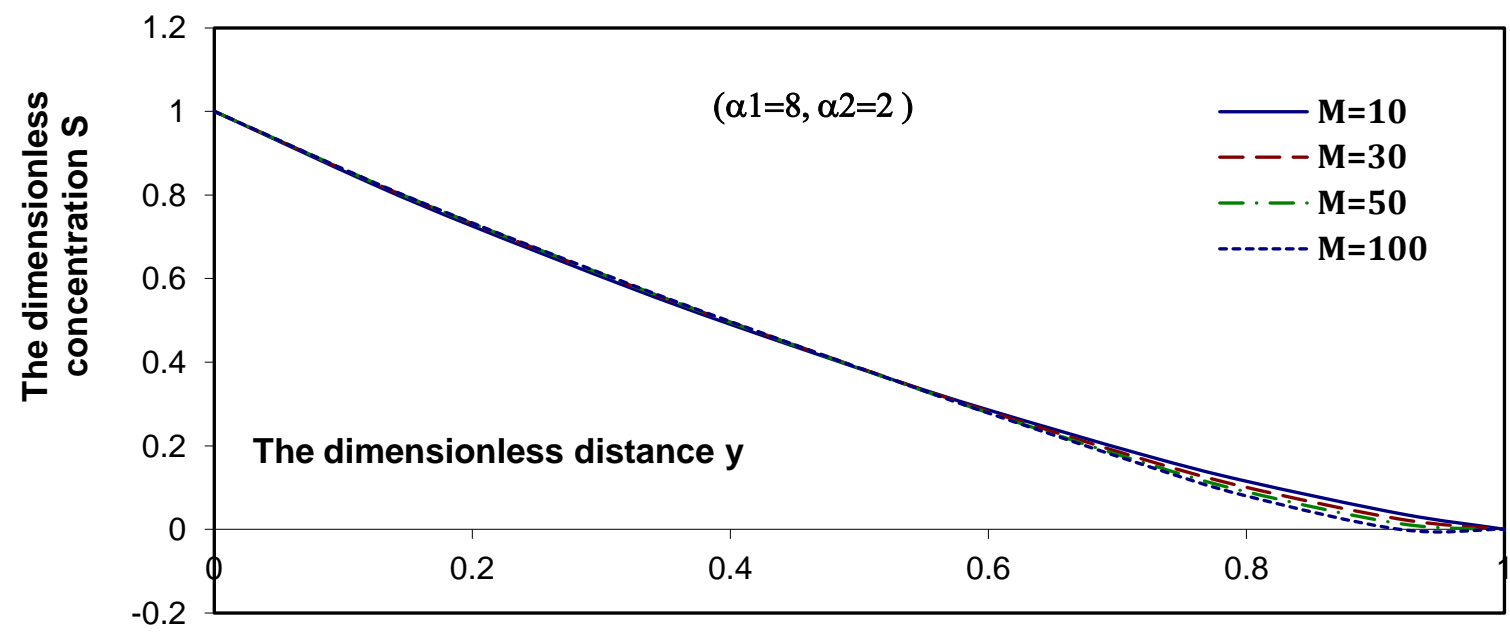

FIG. c-10: Profiles of the concentration $S(y)$ for a pure Couette flow with various values of $M$ for a system have the particulars $\mathrm{Da}=1, \mathrm{Fs}=1, \mathrm{Nt}=0.3, \mathrm{Gr}=0.1, \mathrm{Br}=0.1, \mathrm{Pr}=0.7, \mathrm{Nd}=2, \mathrm{Nb}=0.5, \mathrm{Nt}=0.3, \mathrm{Le}=2, \mathrm{Ld}=0.1, \mathrm{Rc}=0.5, \mathrm{~m}=2$. 


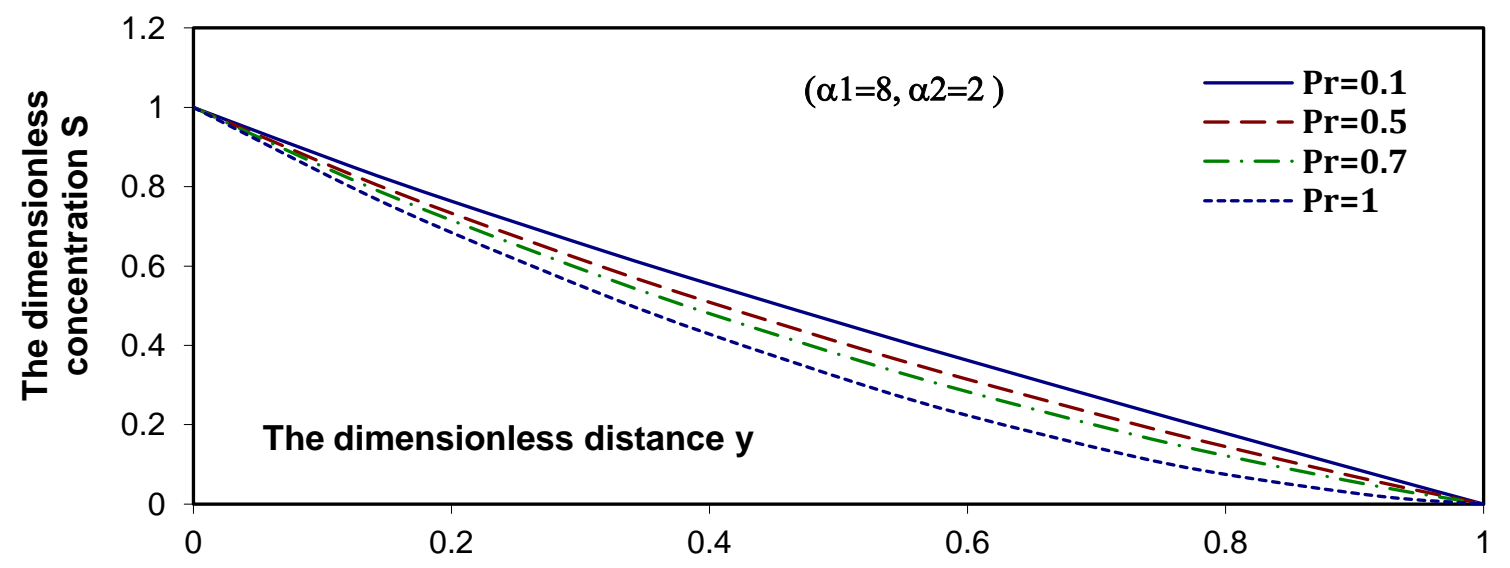

FIG. c-11: Profiles of the concentration $S(y)$ for a pure Couette flow with various values of Pr for a system have the particulars Da=1, Fs=1, Nt=0.3, Gr=0.1, $\mathrm{Br}=0.1, \mathrm{M}=0.1, \mathrm{Nd}=2, \mathrm{Nb}=0.5, \mathrm{Nt}=0.3, \mathrm{Le}=2, \mathrm{Ld}=0.1, \mathrm{Rc}=0.5, \mathrm{~m}=2$.

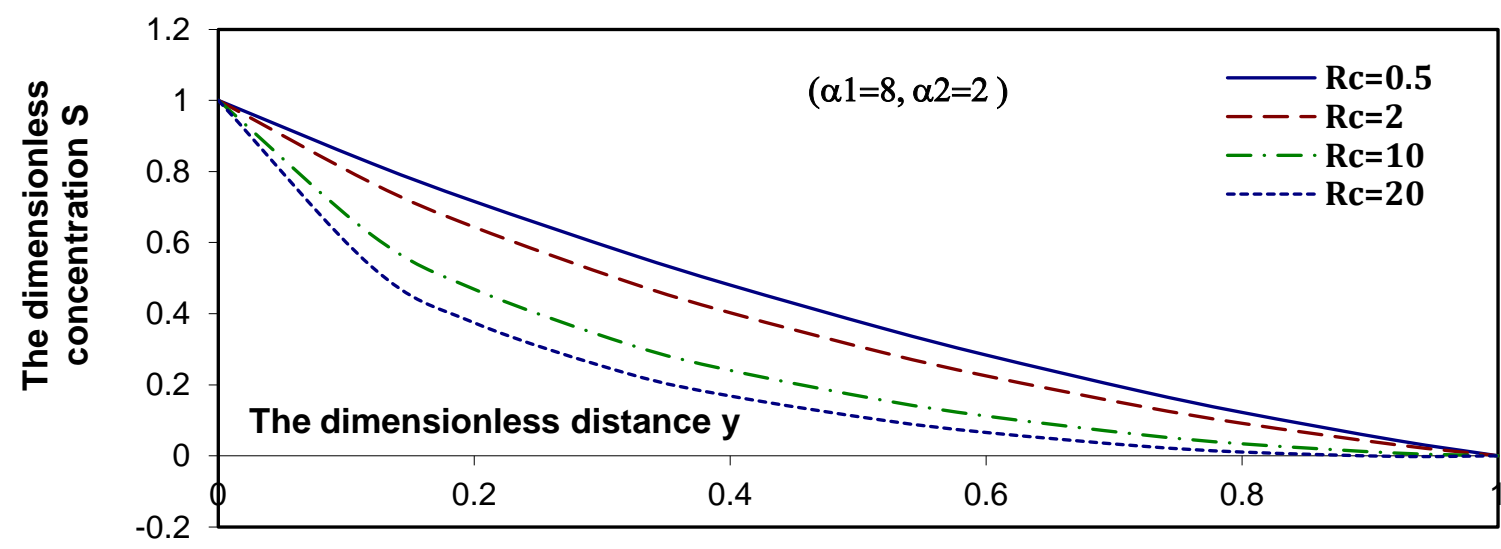

FIG. c-12: Profiles of the concentration $\mathrm{S}(\mathrm{y})$ for a pure Couette flow with various values of $\mathrm{Rc}$ for a system have the particulars $\mathrm{Pr}=0.7, \mathrm{Da}=1, \mathrm{Fs}=1, \mathrm{Nt}=0.3, \mathrm{Gr}=0.1, \mathrm{Br}=0.1, \mathrm{M}=0.1, \mathrm{Nd}=2, \mathrm{Nb}=0.5, \mathrm{Nt}=0.3, \mathrm{Le}=2, \mathrm{Ld}=0.1, \mathrm{~m}=2$.

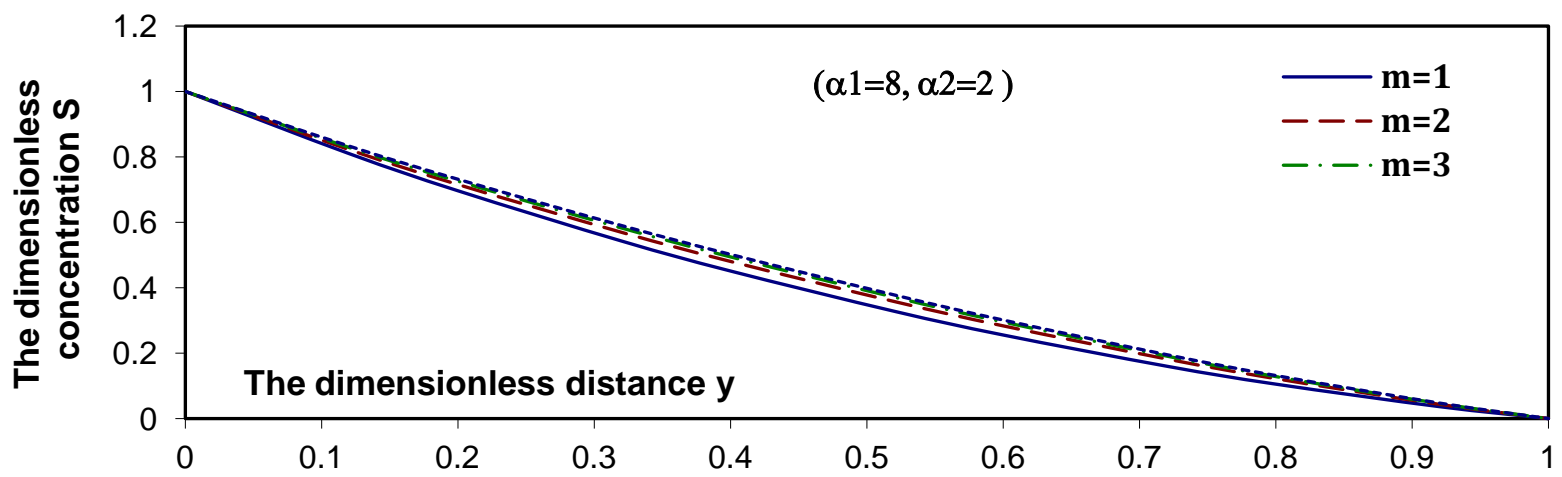

FIG. c-13: Profiles of the concentration $\mathrm{S}(\mathrm{y})$ for a pure Couette flow with various values of $\mathrm{m}$ for a system have the particulars $\operatorname{Pr}=0.7, \mathrm{Da}=1, \mathrm{Fs}=1, \mathrm{Nt}=0.3, \mathrm{Gr}=0.1, \mathrm{Br}=0.1, \mathrm{M}=0.1, \mathrm{Nd}=2, \mathrm{Nb}=0.5, \mathrm{Nt}=0.3, \mathrm{Le}=2, \mathrm{Ld}=0.1, \mathrm{Rc}=0.5$.

Figures (d-1 - d-13) display the distributions of the nanoparticle profile $\boldsymbol{\phi}$ at different values of some parameters of the problem. It is clear that, , the nanoparticle decreases by increasing each of local nanoparticle Grashof $\mathrm{B}_{\mathrm{r}}$, local temperature Grashof number $\mathrm{G}_{\mathrm{r}}$, Sort number $\mathrm{L}_{\mathrm{d}}$, Lewis number $\mathrm{L}_{\mathrm{e}}$, Dufour number $\mathrm{N}_{\mathrm{d}}$, Thermophoresis parameter $\mathrm{N}_{\mathrm{t}}$, Prandtl number $\mathrm{P}_{\mathrm{r}}$, and Chemical reaction parameter $\mathrm{R}_{\mathrm{c}}$. But, the nanoparticle increases by increasing Brownian motion parameter $\mathrm{N}_{\mathrm{b}}$. And, as Darcy number $\mathrm{D}_{\mathrm{a}}$ increases, the nanoparticle decreases in the region $0 \leq y \leq 0.57$ and, it returns increase to $0.57 \leq y \leq 1$. But, as Forchheimer number $\mathrm{F}_{\mathrm{s}}$ increases, the nanoparticle increases in the region $0 \leq y \leq 0.7$ and, it returns decrease to $0.7 \leq y \leq 1$. The effect of magnetic field parameter $\mathrm{M}$ on the nanoparticle disappears in the region $0 \leq y \leq 0.5$ and, the 
nanoparticle returns decrease to $0.5 \leq y \leq 1$ at large values of M. Finally, the nanoparticle increases by increasing Chemical reaction order $\mathrm{m}$.

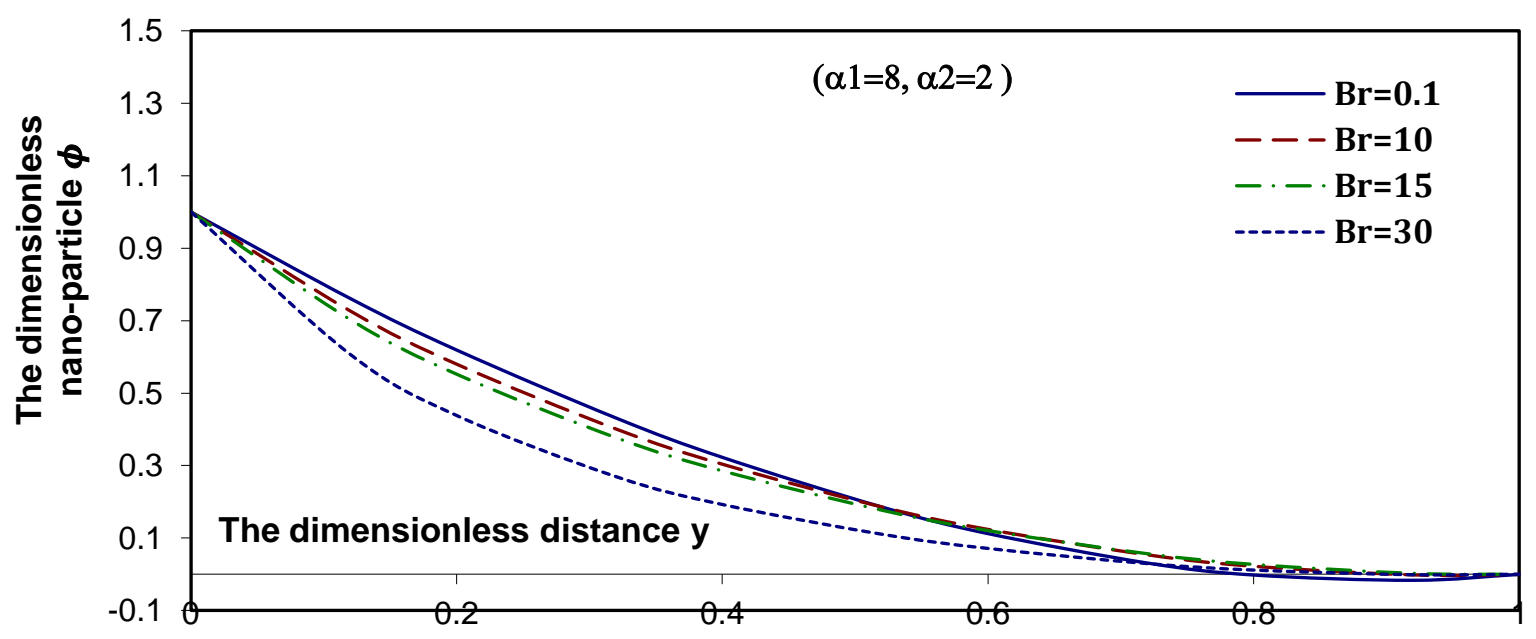

FIG. d-1: Profiles of the nano-particle $\phi(y)$ for a pure Couette flow with various values of $B r$ for a system have the particulars $\mathrm{M}=0.1, \mathrm{Da}=1, \mathrm{Fs}=0.5, \mathrm{Gr}=0.1, \mathrm{Pr}=0.7, \mathrm{Nd}=2, \mathrm{Nb}=0.5, \mathrm{Nt}=0.3, \mathrm{Le}=2, \mathrm{Ld}=0.1, \mathrm{Rc}=0.5, \mathrm{~m}=2$.

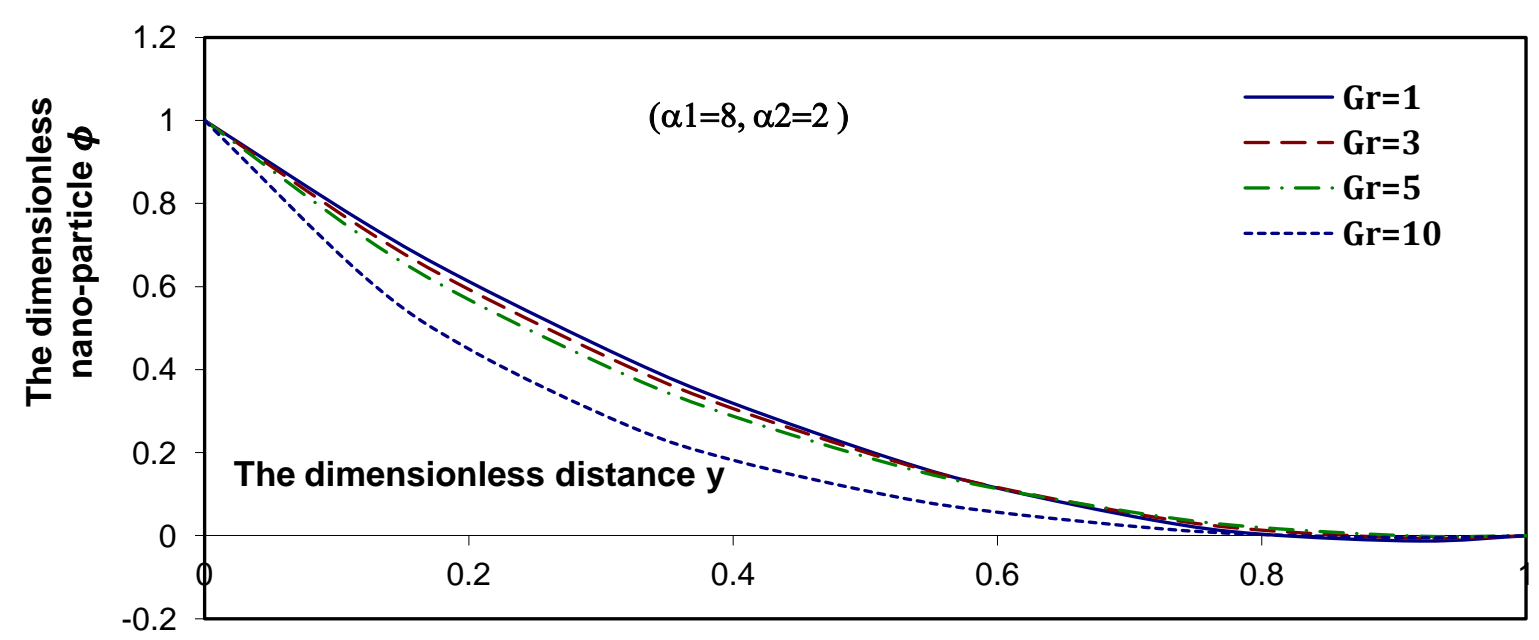

FIG. d-2: Profiles of the nano-particle $\phi(y)$ for a pure Couette flow with various valuesof Gr for a system have the particulars $\mathrm{M}=0.1, \mathrm{Da}=1, \mathrm{Fs}=0.5, \mathrm{Br}=0.1, \mathrm{Pr}=0.7, \mathrm{Nd}=2, \mathrm{Nb}=0.5, \mathrm{Nt}=0.3, \mathrm{Le}=2, \mathrm{Ld}=0.1, \mathrm{Rc}=0.5, \mathrm{~m}=2$.

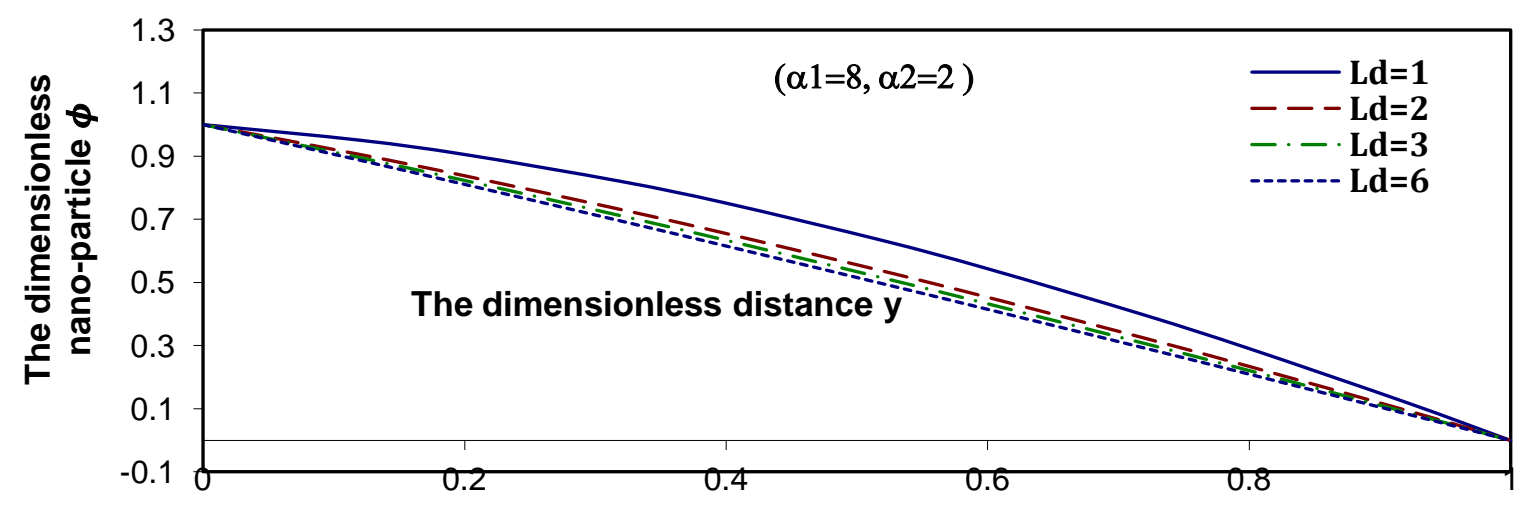

FIG. d-3: Profiles of the nano-particle $\phi(y)$ for a pure Couette flow with various values of Ld for a system have the particulars $\mathrm{M}=0.1, \mathrm{Da}=1, \mathrm{Fs}=0.5, \mathrm{Gr}=0.1, \mathrm{Br}=0.1, \mathrm{Pr}=0.7, \mathrm{Nd}=2, \mathrm{Nb}=0.5, \mathrm{Nt}=0.3, \mathrm{Le}=2, \mathrm{Rc}=0.5, \mathrm{~m}=2$. 


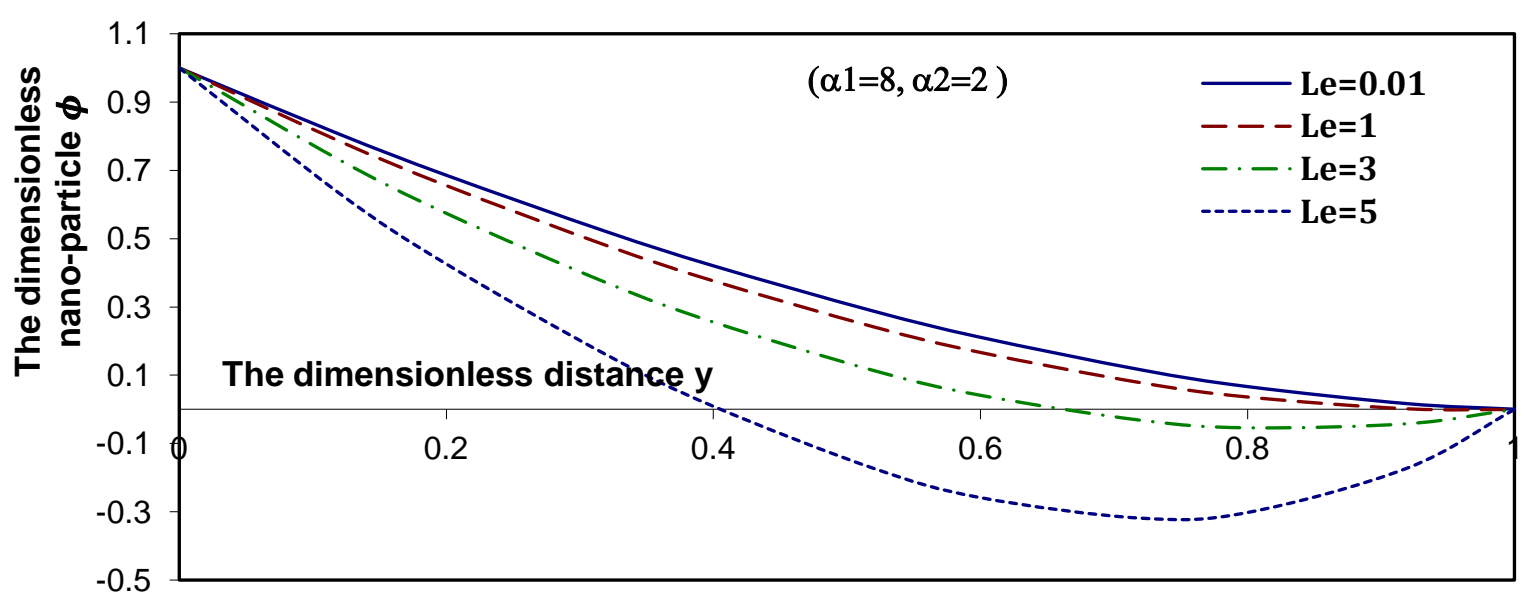

FIG. d-4: Profiles of the nano-particle $\phi(y)$ for a pure Couette flow with various values the Le for a system have the particulars M=0.1, Da=1, Fs=0.5, Gr=0.1, Br=0.1, $\mathrm{Pr}=0.7, \mathrm{Nd}=2, \mathrm{Nb}=0.5, \mathrm{Nt}=0.3, \mathrm{Ld}=0.1, \mathrm{Rc}=0.5, \mathrm{~m}=2$.

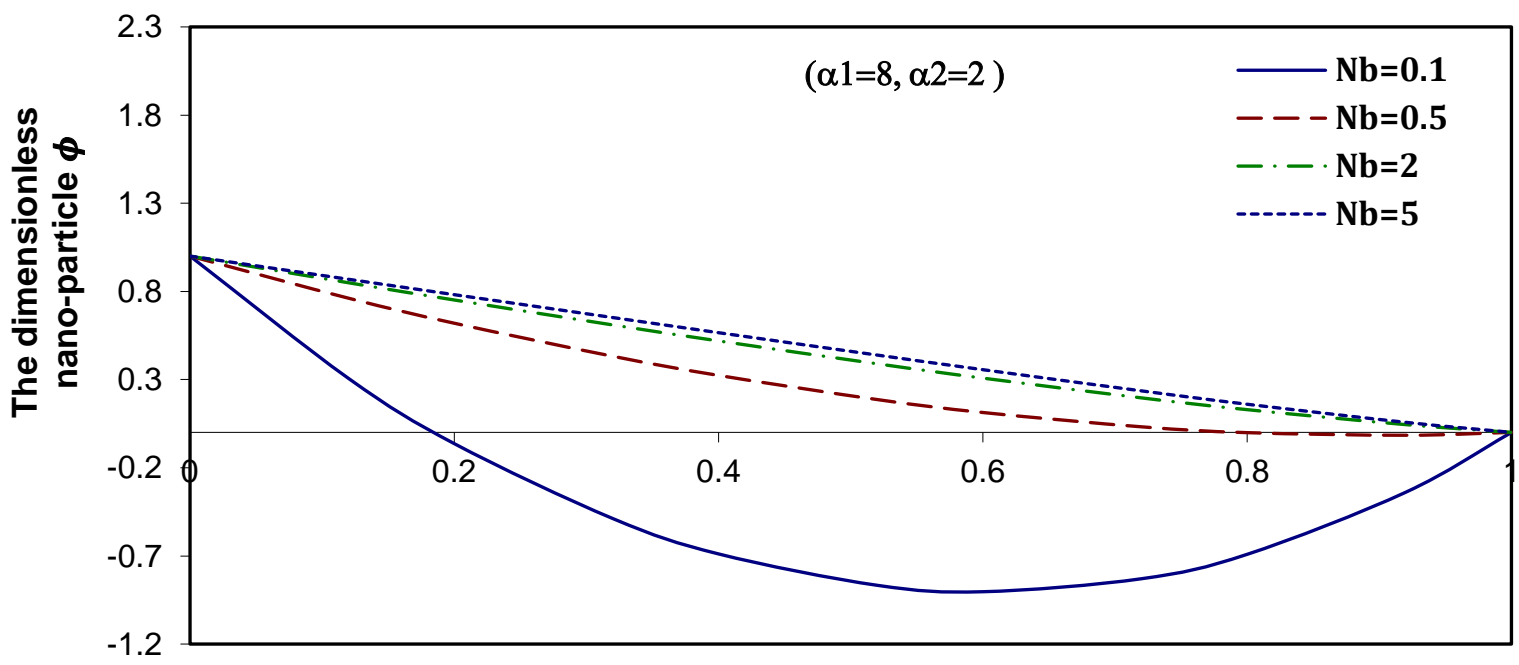

FIG. d-5: Profiles of nano-particle $\phi(y)$ for a pure Couette flow with various values of $\mathrm{Nb}$ for a system have the particulars M=0.1, Da=1, Fs=0.5, Gr=0.1, Br=0.1, Pr=0.7, Nd=2, Le=2, Nt=0.3, Ld=0.1, Rc=0.5, m=2.

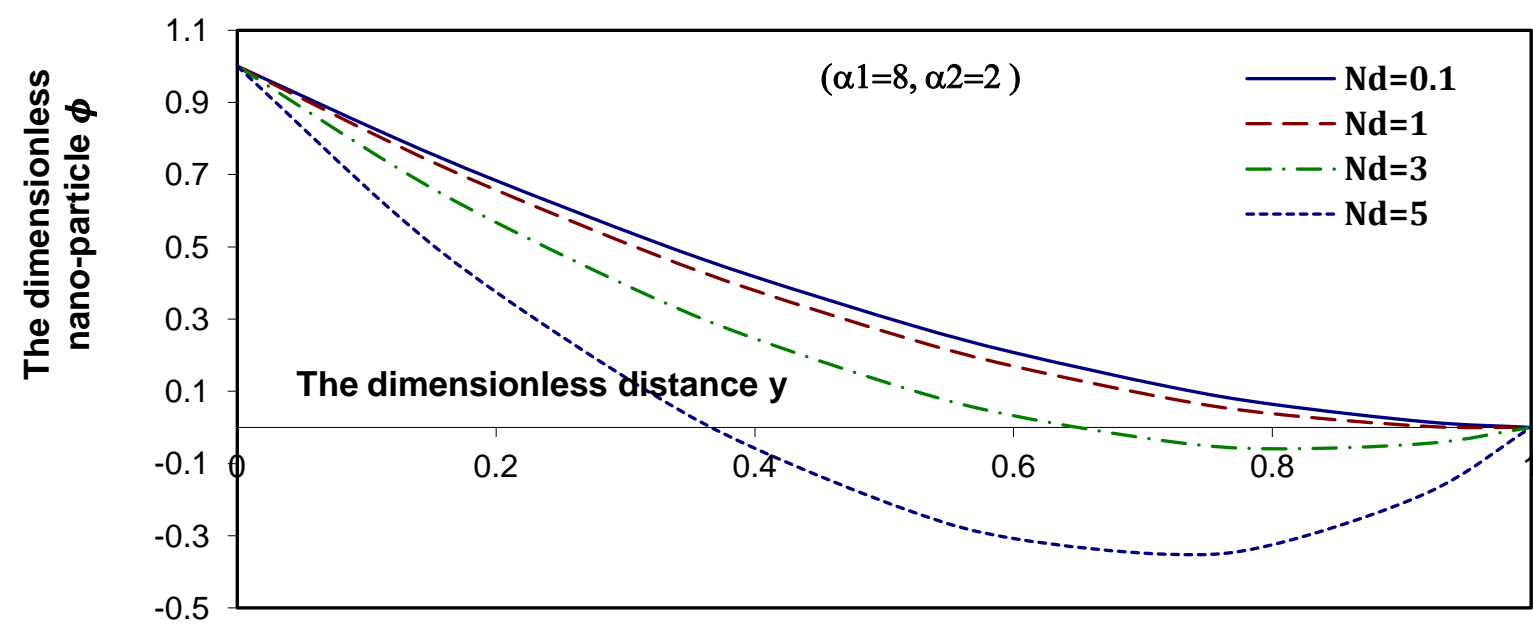

FIG. d-6: Profiles of the nano-particle $\phi(y)$ for a pure Couette flow with various values of Nd for a system have the particulars M=0.1, Da=1, Fs=0.5, Gr=0.1, Br=0.1, Pr=0.7, Nb=0.5, Le=2, Nt=0.3, Ld=0.1, Rc=0.5, m=2. 


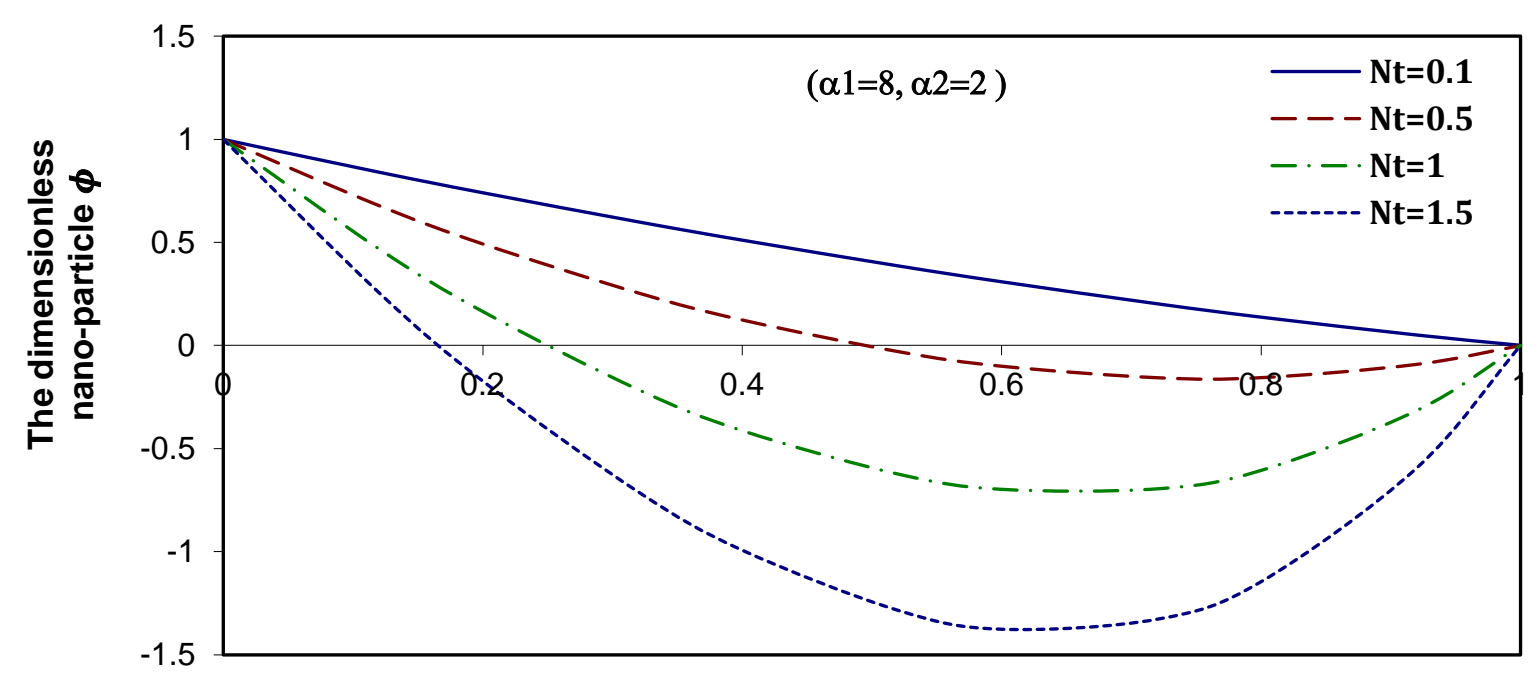

FIG. d-7: Profiles of the nano-particle $\phi(\mathrm{y})$ for a pure Couette flow with various values of $\mathrm{Nt}$ for a system have the particulars M=0.1, Da=1, Fs=0.5, Gr=0.1, Br=0.1, $\mathrm{Pr}=0.7, \mathrm{Nb}=0.5, \mathrm{Le}=2, \mathrm{Nd}=2, \mathrm{Ld}=0.1, \mathrm{Rc}=0.5, \mathrm{~m}=2$.

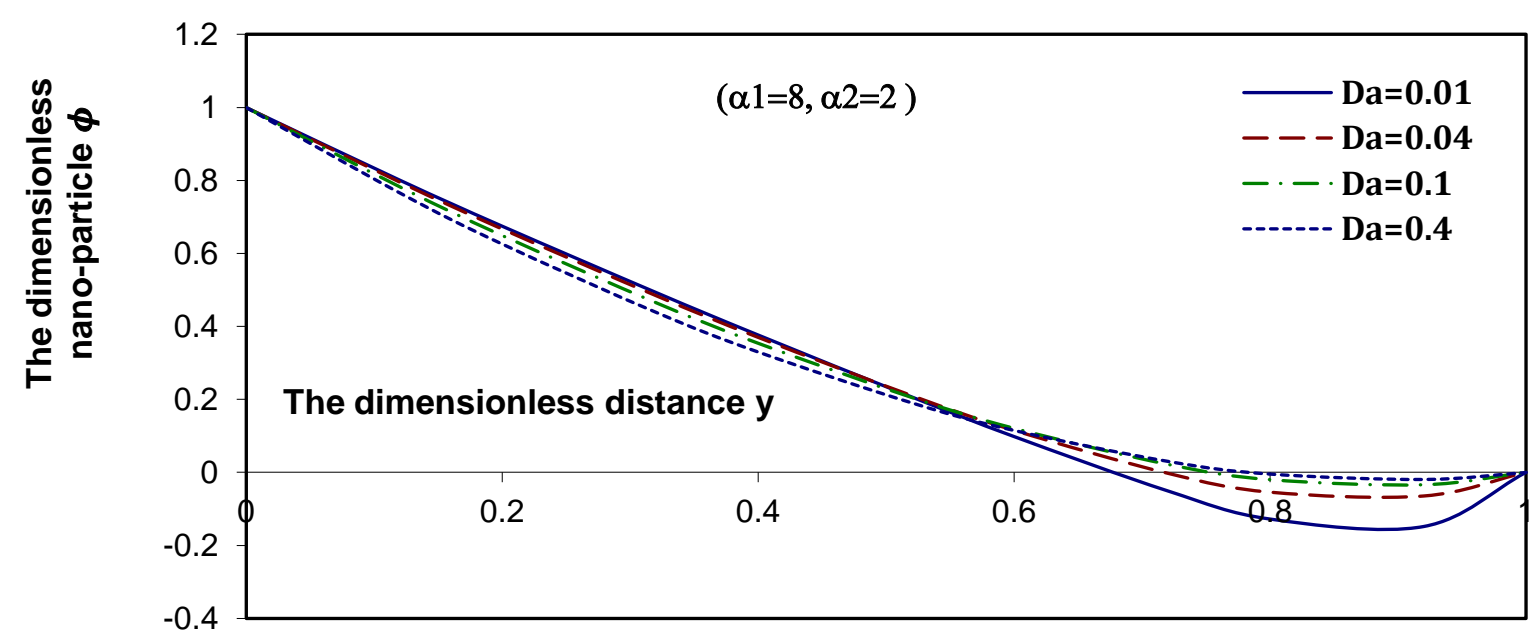

FIG. d-8: Profiles of the nano-particle $\phi(y)$ for a pure Couette flow with various values of Da for a system have the particulars M=0.1, Fs=0.5, Nt=0.3, Gr=0.1, Br=0.1, $\mathrm{Pr}=0.7, \mathrm{Nd}=2, \mathrm{Nb}=0.5, \mathrm{Nt}=0.3, \mathrm{Le}=2, \mathrm{Ld}=0.1, \mathrm{Rc}=0.5, \mathrm{~m}=2$.

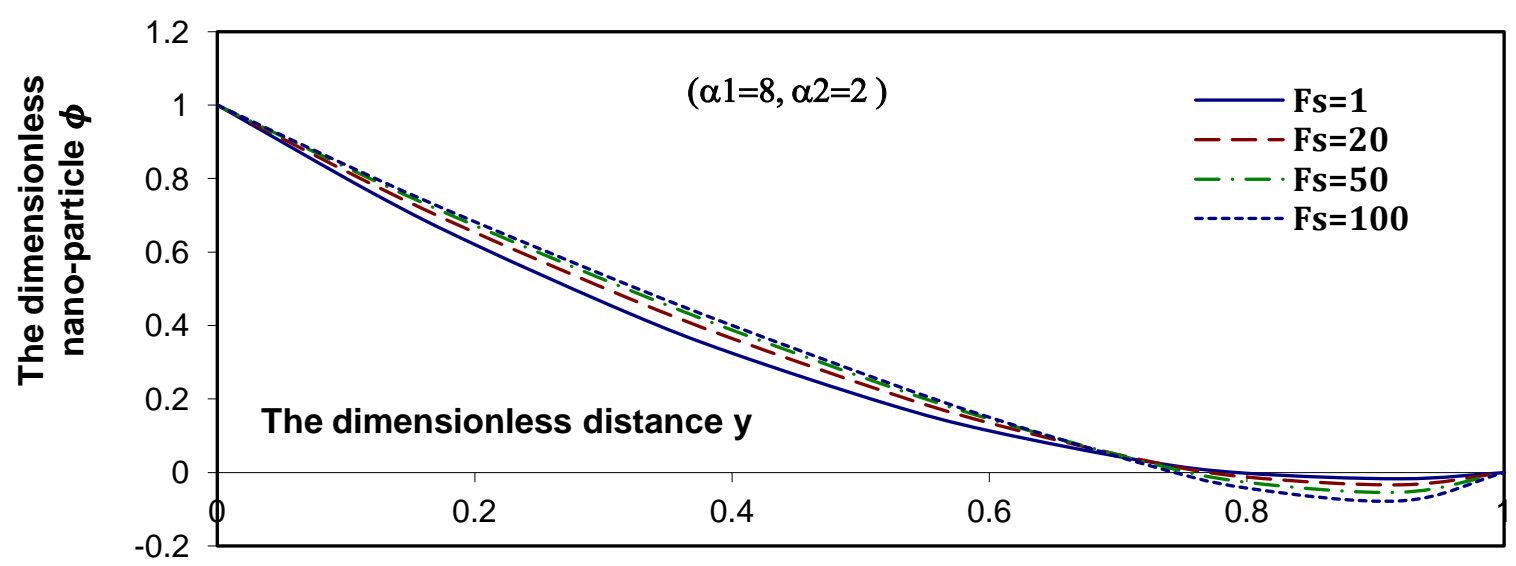

FIG. d-9: Profiles of the nano-particle $\phi(y)$ for a pure Couette flow with various values of Fs for a system have the particulars $\mathrm{M=0.1}, \mathrm{Da}=1, \mathrm{Nt}=0.3, \mathrm{Gr}=0.1, \mathrm{Br}=0.1, \mathrm{Pr}=0.7, \mathrm{Nd}=2, \mathrm{Nb}=0.5, \mathrm{Nt}=0.3, \mathrm{Le}=2, \mathrm{Ld}=0.1, \mathrm{Rc}=0.5, \mathrm{~m}=2$. 


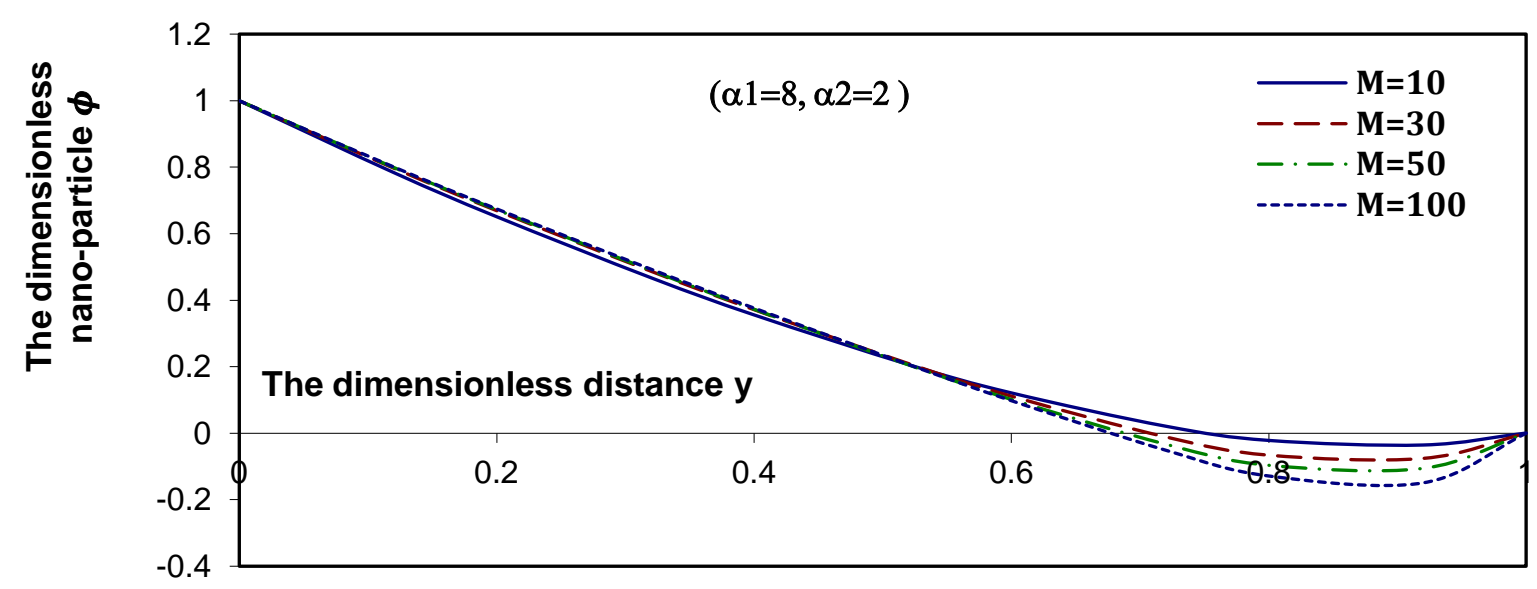

FIG. d-10: Profiles of the nano-particle $\phi(y)$ for a pure Couette flow with various values of $M$ for a system have the particulars $\mathrm{Da}=1, \mathrm{Fs}=0.5, \mathrm{Nt}=0.3, \mathrm{Gr}=0.1, \mathrm{Br}=0.1, \mathrm{Pr}=0.7, \mathrm{Nd}=2, \mathrm{Nb}=0.5, \mathrm{Nt}=0.3, \mathrm{Le}=2, \mathrm{Ld}=0.1, \mathrm{Rc}=0.5, \mathrm{~m}=2$.

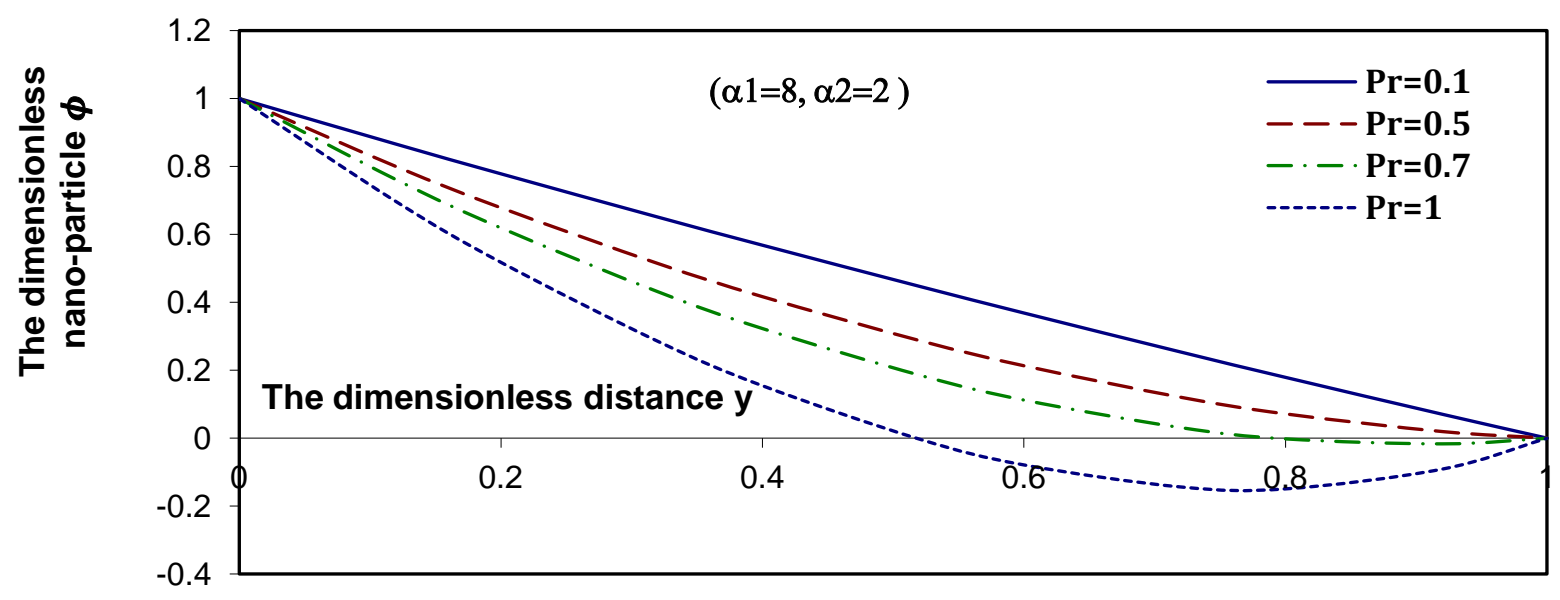

FIG. d-11: Profiles of the nano-particle $\phi(y)$ for a pure Couette flow with various values of $\operatorname{Pr}$ for a system have the particulars $\mathrm{Da}=1, \mathrm{Fs}=0.5, \mathrm{Nt}=0.3, \mathrm{Gr}=0.1, \mathrm{Br}=0.1, \mathrm{M}=0.1, \mathrm{Nd}=2, \mathrm{Nb}=0.5, \mathrm{Nt}=0.3, \mathrm{Le}=2, \mathrm{Ld}=0.1, \mathrm{Rc}=0.5, \mathrm{~m}=2$.

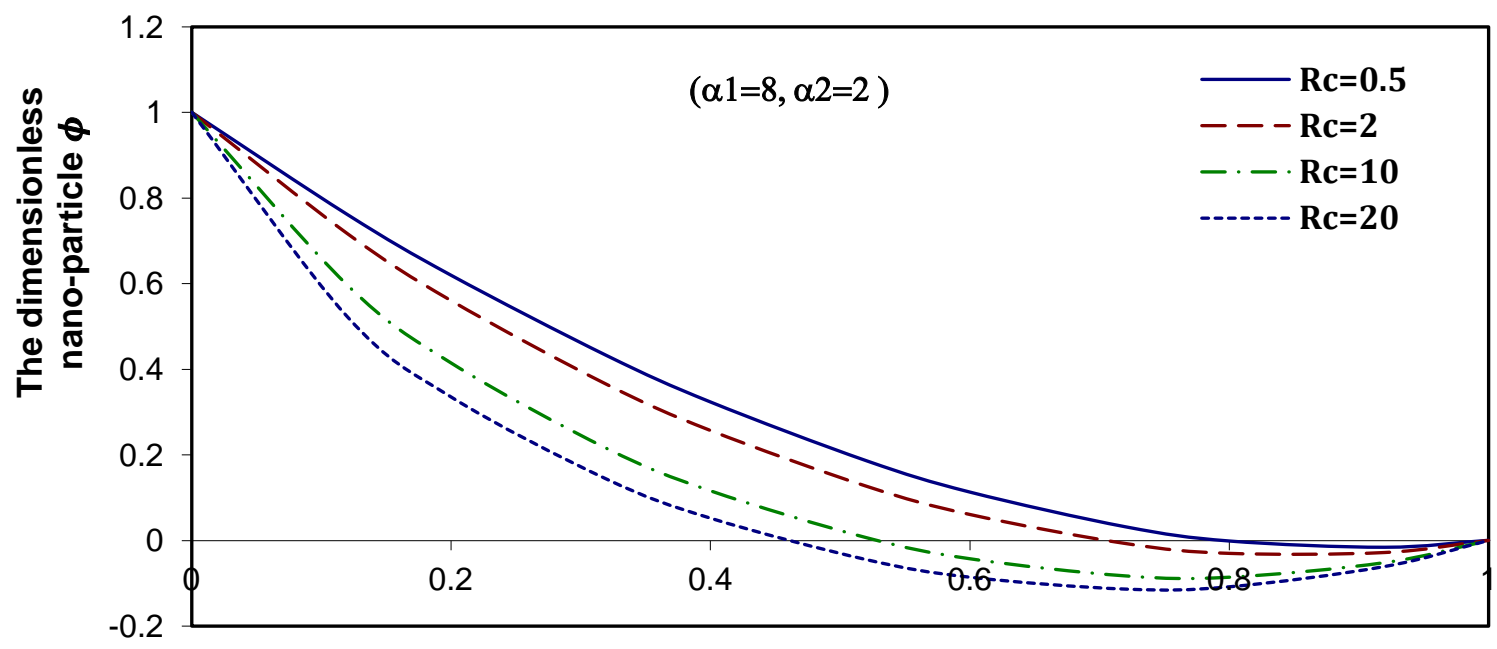

FIG. d-12: Profiles of the nano-particle $\phi(y)$ for a pure Couette flow with various values of Rc for a system have the particulars $\mathrm{Pr}=0.7, \mathrm{Da}=1, \mathrm{Fs}=0.5, \mathrm{Nt}=0.3, \mathrm{Gr}=0.1, \mathrm{Br}=0.1, \mathrm{M}=0.1, \mathrm{Nd}=2, \mathrm{Nb}=0.5, \mathrm{Nt}=0.3, \mathrm{Le}=2, \mathrm{Ld}=0.1, \mathrm{~m}=2$. 


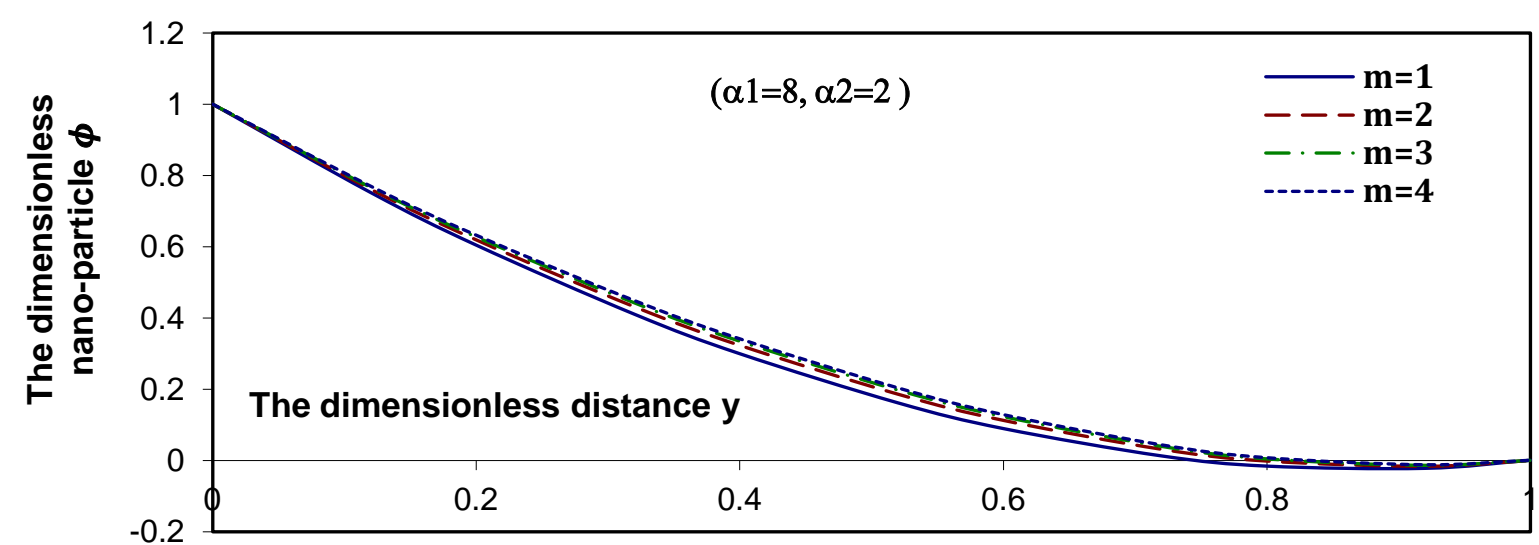

FIG. d-13: Profiles of the nano-particle $\phi(y)$ for a pure Couette flow with various values of $\mathrm{m}$ for a system have the particulars $\mathrm{Pr}=0.7, \mathrm{Da}=1, \mathrm{Fs}=0.5, \mathrm{Nt}=0.3, \mathrm{Gr}=0.1, \mathrm{Br}=0.1, \mathrm{M}=0.1, \mathrm{Nd}=2, \mathrm{Nb}=0.5, \mathrm{Nt}=0.3, \mathrm{Le}=2, \mathrm{Ld}=0.1, \mathrm{Rc}=0.5$.

\subsection{Poiseuille flow}

Figures (e-1 - e-4) describe the effect of pressure gradient $\left(\frac{d \hat{P}}{d x}\right)$ on the velocity, the temperature, the concentration, and the nanoparticle distributions. It is clear that, the velocity, the concentration, and the nanoparticle decrease with increasing $\left(\frac{d \hat{P}}{d x}\right)$, while the temperature increases with increasing $\left(\frac{d \hat{P}}{d x}\right)$.

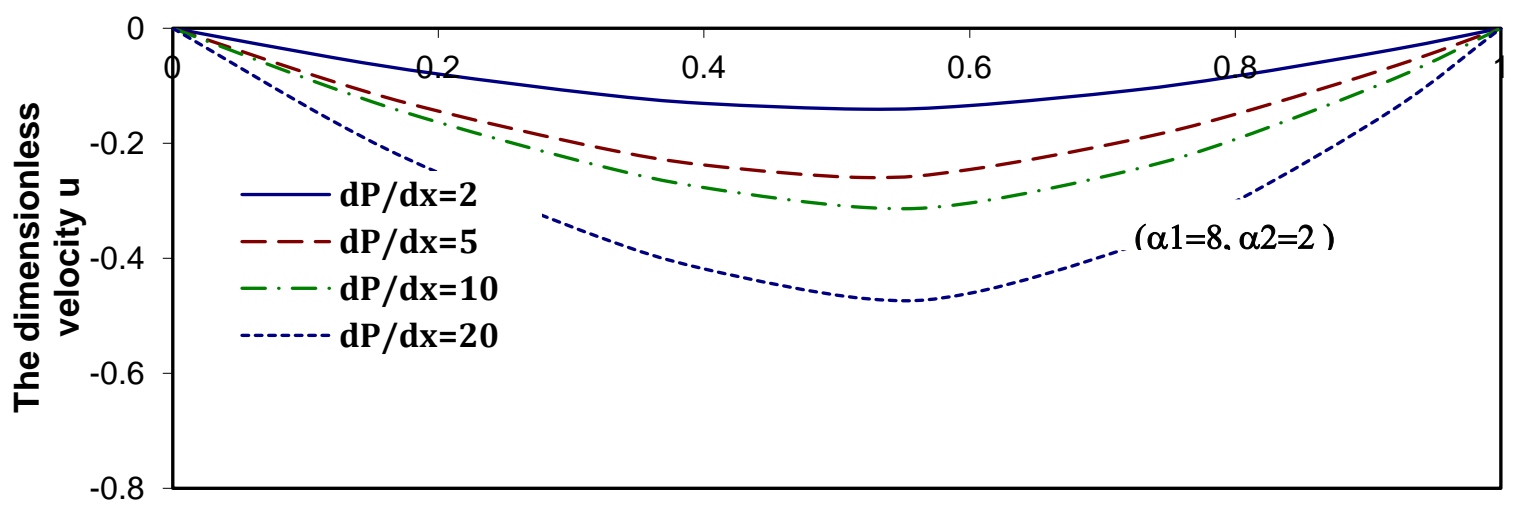

FIG. e-1: Profiles of the velocity $u(y)$ for a pure Poiseuille flow with various values of $\mathrm{dP} / \mathrm{dx}$ for a system have the particulars M=0.1, Da=1, Fs=0.5, Gr=0.1, Br=0.1, Pr=0.7, Nd=2, Nb=0.5, Nt=0.3, Le=2, Ld=0.1, Rc=0.5, m=2.

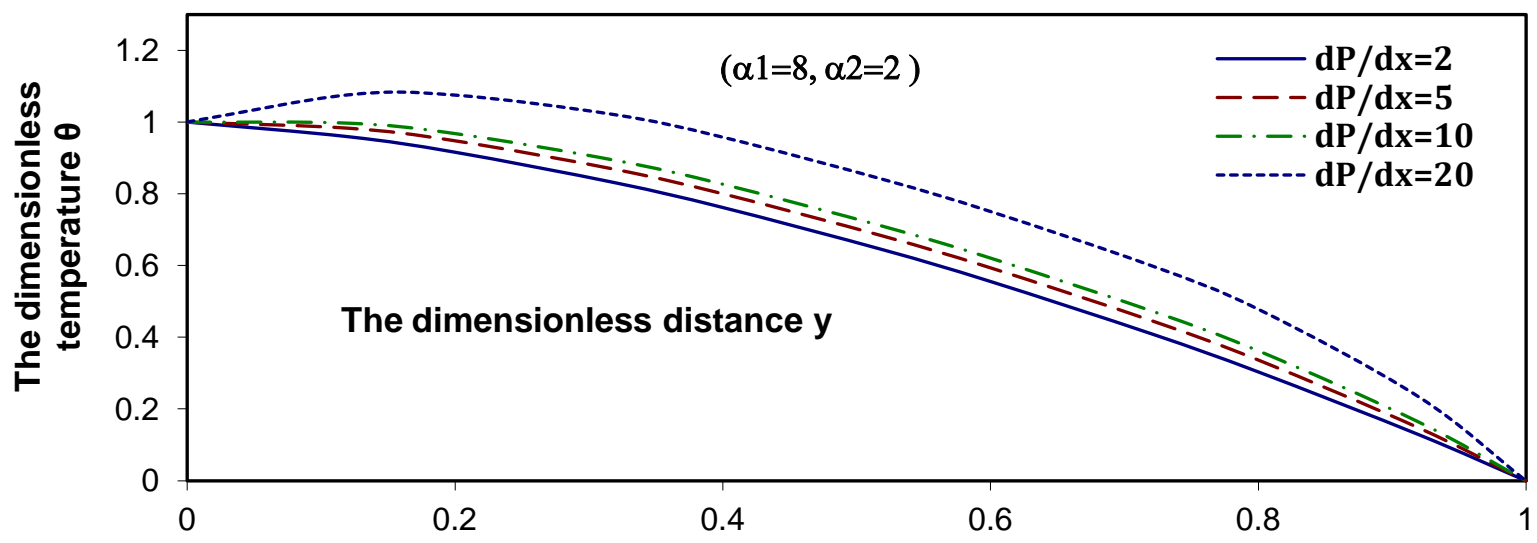

FIG. e-2: Profiles of the temperature $\theta(\mathrm{y})$ for a pure Poiseuille flow with various values of $\mathrm{dP} / \mathrm{dx}$ for a system have the particulars $\mathrm{M}=0.1, \mathrm{Da}=1, \mathrm{Fs}=0.5, \mathrm{Gr}=0.1, \mathrm{Br}=0.1, \mathrm{Pr}=0.7, \mathrm{Nd}=2, \mathrm{Nb}=0.5, \mathrm{Nt}=0.3, \mathrm{Le}=2, \mathrm{Ld}=0.1, \mathrm{Rc}=0.5, \mathrm{~m}=2$. 


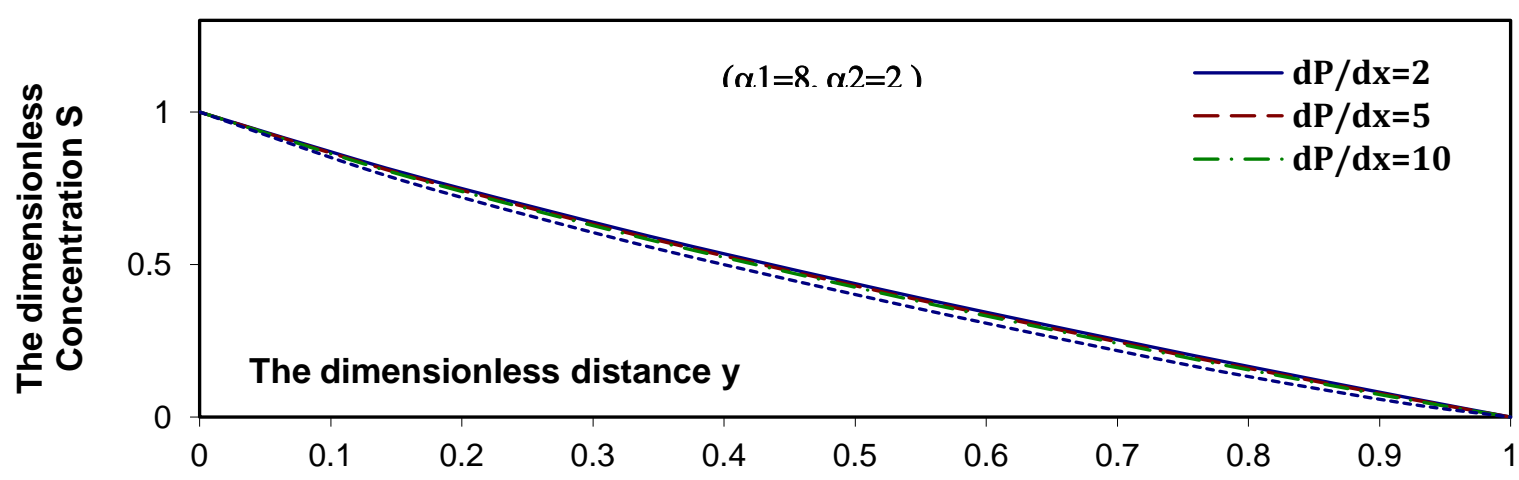

FIG. e-3: Profiles of the concentration $\mathrm{S}(\mathrm{y})$ for a pure Poiseuille flow with various values of $\mathrm{dP} / \mathrm{dx}$ for a system have the particulars $\mathrm{M}=0.1, \mathrm{Da}=1, \mathrm{Fs}=0.5, \mathrm{Gr}=0.1, \mathrm{Br}=0.1, \mathrm{Pr}=0.7, \mathrm{Nd}=2, \mathrm{Nb}=0.5, \mathrm{Nt}=0.3, \mathrm{Le}=2, \mathrm{Ld}=0.1, \mathrm{Rc}=0.5, \mathrm{~m}=2$.

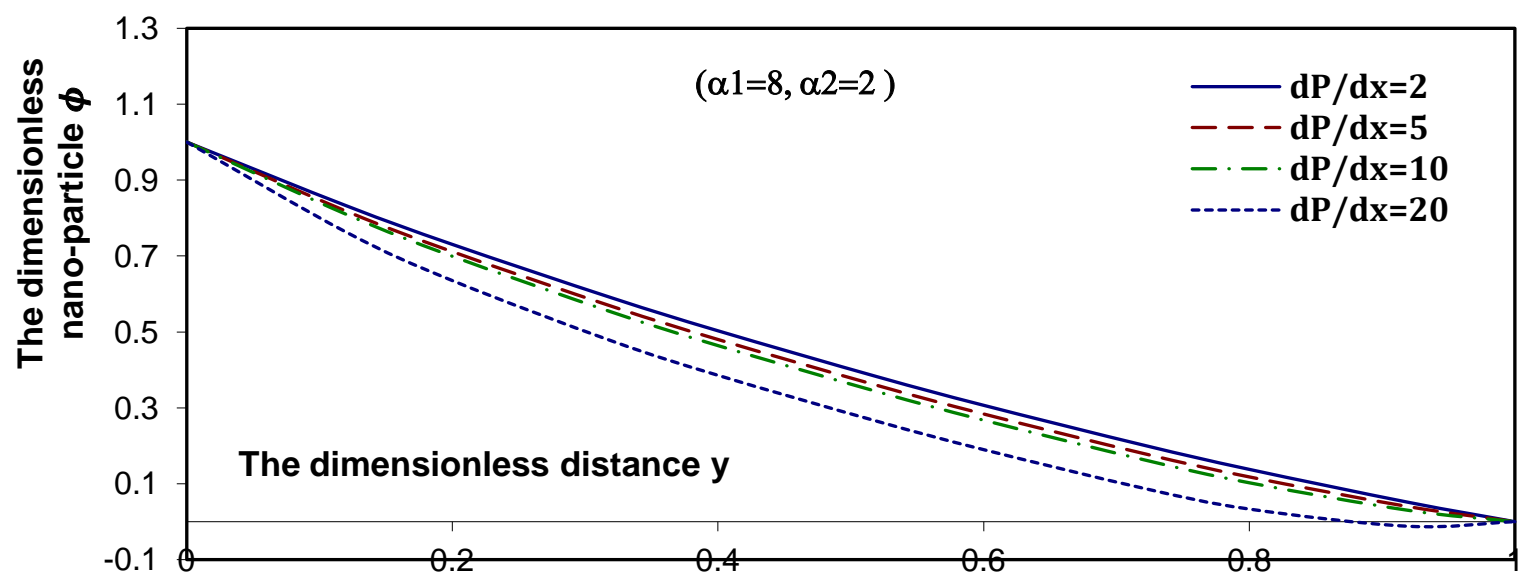

FIG. e-4: Profiles of the nano-particle $\phi(\mathrm{y})$ for a pure Poiseuille flow with various values of $\mathrm{dP} / \mathrm{dx}$ for a system have the particulars $\mathrm{M}=0.1, \mathrm{Da}=1, \mathrm{Fs}=0.5, \mathrm{Gr}=0.1, \mathrm{Br}=0.1, \mathrm{Pr}=0.7, \mathrm{Nd}=2, \mathrm{Nb}=0.5, \mathrm{Nt}=0.3, \mathrm{Le}=2, \mathrm{Ld}=0.1, \mathrm{Rc}=0.5, \mathrm{~m}=2$.

8.3

Generalized Couette flow

If the flow of an Oldroyd 6-constant fluid between two parallel plates is driven by both the motion of the top plate and a constant pressure gradient in the direction parallel to the plates, a generalized couette flow is formed for $\left(\frac{d \hat{P}}{d x} \neq 0\right.$, $\left.\mathrm{U}_{0}=1\right)$. Numerical results for such a generalized couette flow are illustrated in figures (f-1 - f-4).

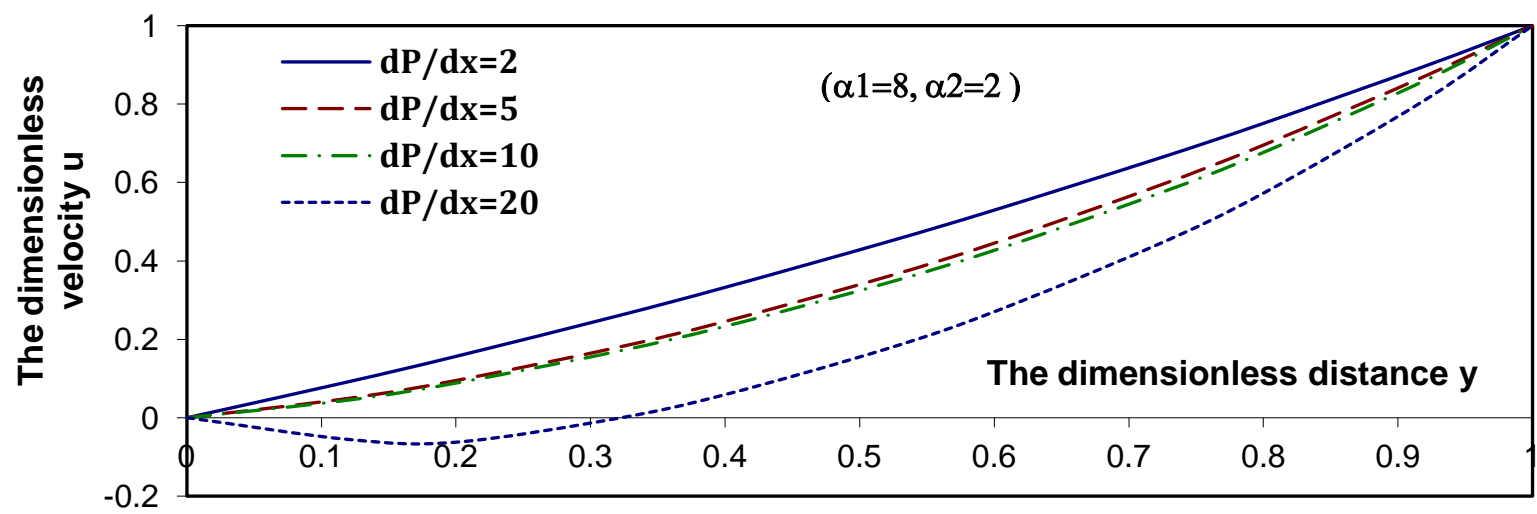

FIG. f-1: Profiles of the velocity $u(y)$ for a generalized Couette flow with various values of $\mathrm{dP} / \mathrm{dx}$ for a system have the particulars $\mathrm{M}=0.1, \mathrm{Da}=1, \mathrm{Fs}=0.5, \mathrm{Gr}=0.1, \mathrm{Br}=0.1, \mathrm{Pr}=0.7, \mathrm{Nd}=2, \mathrm{Nb}=0.5, \mathrm{Nt}=0.3, \mathrm{Le}=2, \mathrm{Ld}=0.1, \mathrm{Rc}=0.5, \mathrm{~m}=2$. 


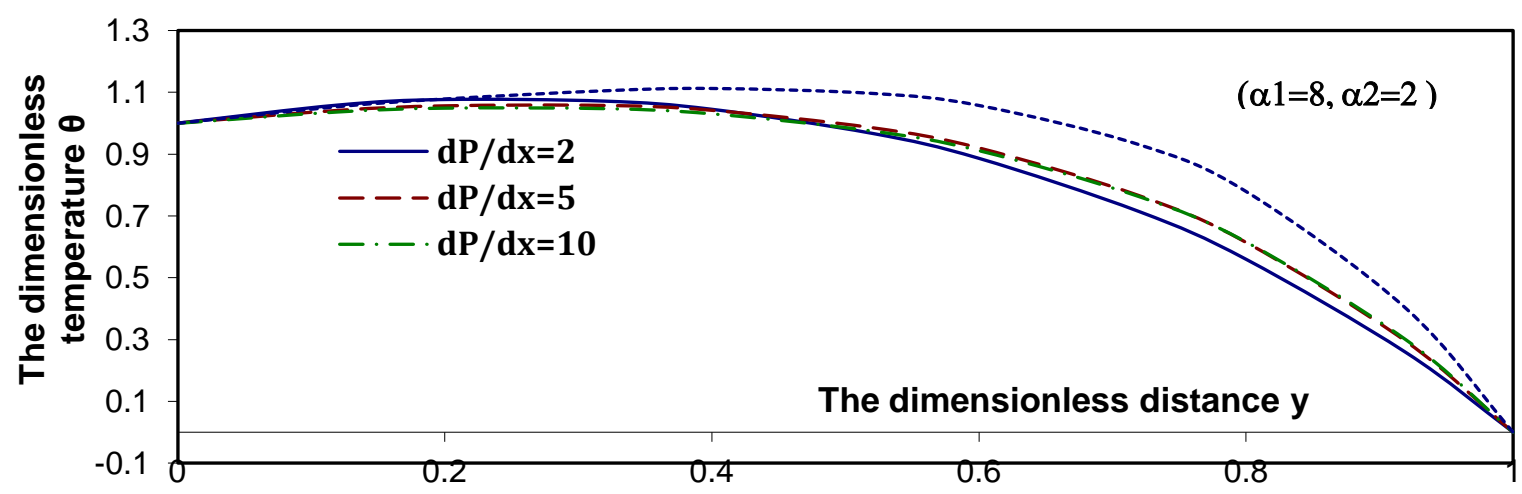

FIG. f-2: Profiles of the temperature $\theta(y)$ for a generalized Couette flow with various values of $\mathrm{dP} / \mathrm{dx}$ for a system have the particulars $\mathrm{M}=0.1, \mathrm{Da}=1, \mathrm{Fs}=0.5, \mathrm{Gr}=0.1, \mathrm{Br}=0.1, \mathrm{Pr}=0.7, \mathrm{Nd}=2, \mathrm{Nb}=0.5, \mathrm{Nt}=0.3, \mathrm{Le}=2, \mathrm{Ld}=0.1, \mathrm{Rc}=0.5, \mathrm{~m}=2$.

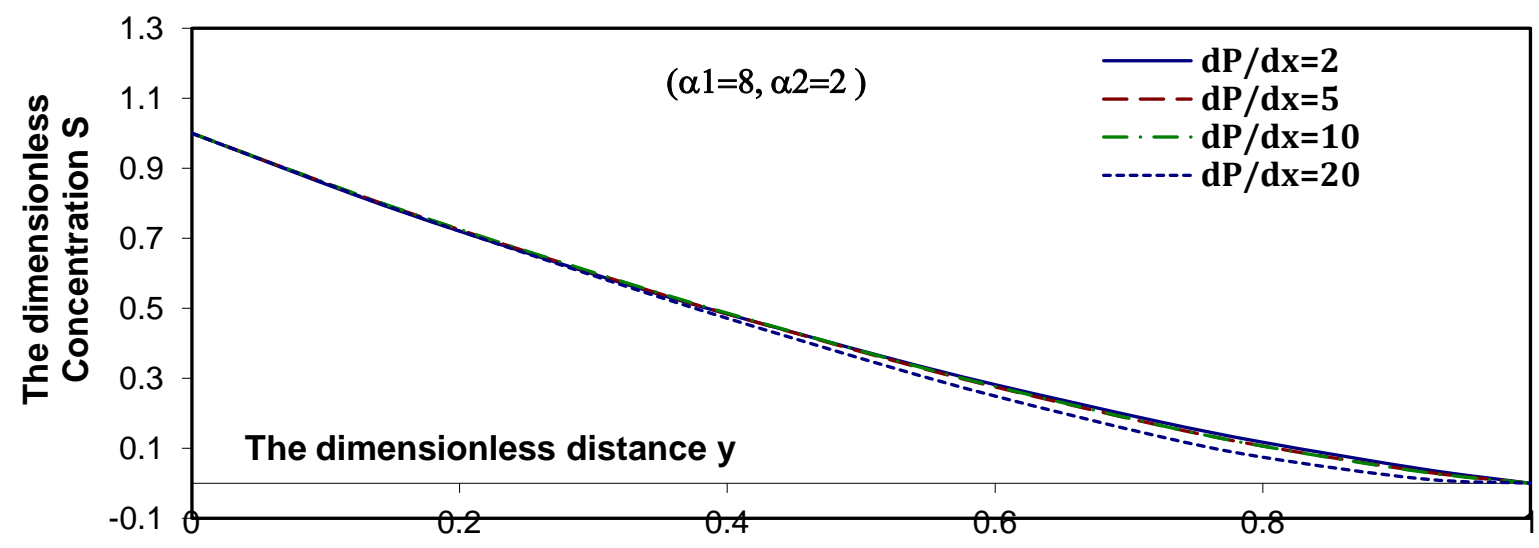

FIG. f-3: Profiles of the concentration $\mathrm{S}(\mathrm{y})$ for a generalized Couette flow with various values of $\mathrm{dP} / \mathrm{dx}$ for a system have the particulars $\mathrm{M}=0.1, \mathrm{Da}=1, \mathrm{Fs}=0.5, \mathrm{Gr}=0.1, \mathrm{Br}=0.1, \mathrm{Pr}=0.7, \mathrm{Nd}=2, \mathrm{Nb}=0.5, \mathrm{Nt}=0.3, \mathrm{Le}=2, \mathrm{Ld}=0.1, \mathrm{Rc}=0.5, \mathrm{~m}=2$.

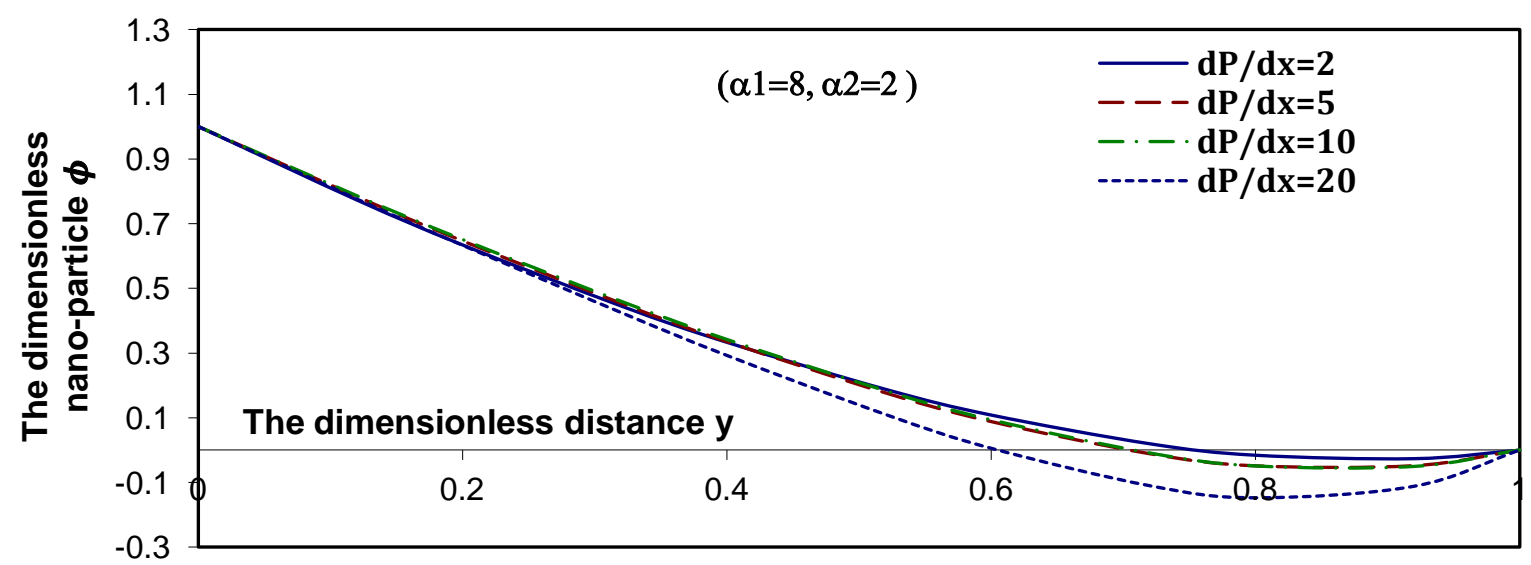

FIG. f-4: Profiles of the nano-particle $\phi(\mathrm{y})$ for a generalized Couette flow with various values of $\mathrm{dP} / \mathrm{dx}$ for a system have the particulars M=0.1, Da=1, Fs=0.5, Gr=0.1, Br=0.1, Pr=0.7, Nd=2, Nb=0.5, Nt=0.3, Le=2, Ld=0.1, Rc=0.5, $\mathrm{m}=2$.

Figures (f-1 - f-4) display the variation of the velocity, the temperature, the concentration, and the nanoparticle distributions for several values of the pressure gradient for $\left(\frac{d \hat{P}}{d x}\right)$. It is observed that, the velocity, the concentration, and the nanoparticle decrease with increasing $\left(\frac{d \hat{P}}{d x}\right)$, while the temperature increases with increasing $\left(\frac{d \hat{P}}{d x}\right)$. 


\section{CONCLUSION}

In this work, we have studied magnetohydrodynamic flows of an Oldroyd 6-constant nano-fluid through a non-Darcy porous medium with heat and mass transfer. The governing boundary value problem was solved numerically by an Explicit FiniteDifference method. We concentrated our work on obtaining the velocity, the temperature, the concentration, and the nanoparticle distributions which are illustrated graphically at different values of the parameters of the problem in three cases, pure Couette flow, pure Poiseuille flow, and generalized Couette flow. Global error estimation is also obtained using Zadunaisky technique. We used 26 points to find the interpolating polynomial of degree 25 in interval $[0,1]$ and the results are shown in table (1). We notice that, the error in table (1) is good enough to justify the use of resulting numerical values. The main findings from the current study can be summarized as follows:

1- By increasing $B_{r}, G_{r}$, and $D_{a}$, the velocity $u$ increases, whereas it decreases by increasing each of $F_{s}$, and $M$ in the case of Couette flow at $\left(\alpha_{1}=8, \alpha_{2}=2\right)$. And, the other parameters doesn't have any effect on the velocity.

2- By increasing $B_{r}, G_{r}$, and $D_{a}$, the temperature $\theta$ increases at the first part $(0 \leq y \leq 0.58)$ while, it decreases at the second part $(0.58 \leq y \leq 1)$. And, by increasing $\mathrm{L}_{\mathrm{d}}, \mathrm{L}_{\mathrm{e}}, \mathrm{N}_{\mathrm{b}}, \mathrm{N}_{\mathrm{d}}, \mathrm{N}_{\mathrm{t}}, \mathrm{P}_{\mathrm{r}}$, and $\mathrm{R}_{\mathrm{c}}$ the temperature increases, while by increasing $\mathrm{m}$, the temperature decreases. By increasing $\mathrm{F}_{\mathrm{s}}$, the temperature decreases at the first part $(0 \leq y \leq 0.7)$ while, it increases at the second part $(0.7 \leq y \leq 1)$. Also, By increasing $\mathrm{M}$, the temperature decreases at the first part $(0 \leq y \leq 0.5)$ while, it increases at the second part $(0.5 \leq y \leq 1)$. All these results are given in the case of Couette flow at $\left(\alpha_{1}=8, \alpha_{2}=2\right)$.

3- By increasing $\mathrm{Br}, \mathrm{Gr}, \mathrm{Ld}, \mathrm{Le}, \mathrm{Nb}, \mathrm{Nd}, \mathrm{Nt}, \mathrm{Pr}$, and $\mathrm{Rc}$, the concentration $\mathrm{S}$ decreases, while by increasing $\mathrm{m}$, the concentration increases. By increasing $\mathrm{Da}$, the concentration decreases at the first part $(0 \leq y \leq 0.6)$ while, it increases for $(0.6 \leq y \leq 1)$. But, by increasing Fs, the concentration increases in the first part $(0 \leq y \leq 0.7)$ while, it decreases at the second part $(0.7 \leq y \leq 1)$. And, by increasing $\mathrm{M}$, the concentration doesn't have any change at the first part $(0 \leq y \leq 0.6)$ while, it decreases at the second part $(0.6 \leq y \leq 1)$ at large values of M. All these results are given in the case of Couette flow at $\left(\alpha_{1}=8, \alpha_{2}=2\right)$.

4- By increasing $B_{r}, G_{r}, L_{d}, L_{e}, N_{d}, N_{t}, P_{r}$ and $R_{c}$, the nanoparticle $\phi$ decreases, while by increasing $N_{b}$, and $m$, the nanoparticle increases. By increasing $\mathrm{Da}$, the nanoparticle decreases at the first part $(0 \leq y \leq 0.57)$ while, it increases for $(0.57 \leq y \leq 1)$. But, by increasing Fs, the nanoparticle increases in the first part $(0 \leq y \leq 0.7)$ while, it decreases at the second part $(0.7 \leq y \leq 1)$. And, by increasing $M$, the nanoparticle doesn't have any change at the first part $(0 \leq y \leq 0.5)$ while, it decreases at the second part $(0.5 \leq y \leq 1)$ at large values of $\mathrm{M}$. All these results are given in the case of Couette flow at $\left(\alpha_{1}=8, \alpha_{2}=2\right)$.

5- By increasing $\frac{d \hat{P}}{d x}$, the velocity, the concentration, and the nanoparticle decrease, while the temperature increases in the case of Poiseuille flow at $\left(\alpha_{1}=8, \alpha_{2}=2\right)$.

6- By increasing $\frac{d \hat{P}}{d x}$, the velocity, the concentration, and the nanoparticle decrease, while the temperature increases in the case of generalized Couette flow at $\left(\alpha_{1}=8, \alpha_{2}=2\right)$.

\section{REFERENCES}

[1] A. Mushtaq, M. Mustafa, T. Hayat, and A. Alsaedi, "Nonlinear radiative heat transfer in the flow of nanofluid due to solar energy: A numerical study", J. Taiwan Inst. Chem. Eng., Vol. 45, Pp. 1176-1183, 2014.

[2] C. Guillope, and JC. Saut, "Global existence and one-dimensional non-linear stability of shearing motions of viscoelastic fluids of Oldroyd type, RAIRO Model", Math Anal Numer, Vol. 24, Pp. 369-401, 1990.

[3] F. Trieb, and J. Nitsch, "Recommendations for the market introduction of solar thermal power stations, Renew Energy", Vol. 14, Pp. 17-22, 1998.

[4] G. Pontrelli, "Longitudinal and torsional oscillations of a rod in an Oldroyd-B fluid with suction or injection", Acta Mech. , Vol. 123, Pp. 57-68, 1997.

[5] G. Pontrelli, and K. Bhatnagar, "Flow of a viscoelastic fluid between two rotating circular cylinders subject to suction or injection", Int. J. Numer. Maths. Fluids, Vol. 24, Pp. 337-349, 1997.

[6] H. C. Saxena, "Examples in finite differences and numerical analysis:, S. Chand \& Company Ltd, Ram Nagar, New delhi, 1991.

[7] H. Masuda, A. Ebata, K. Teramae, and N. Hishinuma, (1993), Alteration of thermal conductivity and viscosity of liquid by dispersing ultra-fine particles, Netsu Bussei, Vol. 7: 227-233. 
[8] J. Bear, (1972), Dynamics of fluids in Porous media, Elsevier, New York.

[9] J. Bungiorno, (2006), Convective transport in nanofluids, ASME J. Heat Transfer, Vol. 128: 240-250.

[10] J. N. Kapur, (1973), An Introduction of fluid dynamics, Indian Institute of Technology.

[11] JG. Oldroyd, (1951), The motion of an elastic-viscous liquid contained between coaxial cylinders, Quart. J. Mech. Appl. Math., Vol. 4: $271-282$

[12] K. Vajravelu, K. V. Prasad, and S. Abbasbandy, (2013), Convection transport of nanoparticle in multi-layer fluid flow, Appl. Math. Mech. Eng. Ed., Vol. 34(2): 177-188.

[13] KR. Rajagopal, (1996), On an exact solution for the flow of Oldroyd-B fluid, Bull Tech. Univ. Istanbul, Vol. 49: 617-623.

[14] M. A. Rana, A. M. Siddiqui, and R. Qamar, (2009), Hall effects on hydromagnetic flow of an Oldroyd 6-constant fluid between concentric cylinders, Chaos, Solitons and Fractals, Vol. 39: 204-213.

[15] Md. Shakhaoath Khan, Ifsana Karim, and Md. Sirajul Islam, (2014), Possessions of chemical reaction on MHD Heat and Mass Transfer Nanofluid Flow on a Continuously Moving Surface, Amer. Chem. Sci. J., Vol. 4(3): 401-415.

[16] Mohamed Y. Abou-zeid, Abeer A. Shaaban and Muneer Y. Alnour, (2015), Numerical treatment and global error estimation of natural convective effects on gliding motion of bacteria on a power-law nanoslime through a non-Darcy porous medium, J. Porous Media, Vol. 18(11): 1091-1106.

[17] Nabil T.M. El-dabe, Abeer A. Shaaban, Mohamed Y. Abou-zeid, and Hemat A. Ali, ( 2015), MHD non-Newtonian nanofluid flow over a stretching sheet with thermal diffusion and diffusion thermo effects through a non-Darcy porous medium with radiation and chemical reaction, J. Compu. Theor. Nanoscience, Vol. 12(11):1-9.

[18] P. A. Davidson, (2001), An Introduction to magnetohydrodynamics, Cambridge University Press.

[19] P. E. Zadunaisky, (1976), On the Estimation of Errors Propagated in the Numerical Integration of Ordinary Differential Equations, Numer. Math., Vol. 27: 21-29.

[20] R. M. Saldanha da Gama, (2005), A new mathematical modelling for describing the unsaturated flow of an incompressible liquid through a rigid porous medium, Int. J. Non-Linear Mec., Vol. 40: 49-68.

[21] S. Baris, (2001), Flow of an Oldroyd 6-constant fluid between intersecting planes, one of which is moving, Acta Mech. Vol. 147: 128135 .

[22] S. Choi, (2009), Enhancing thermal conductivity of fluids with nanoparticles, In: Siginer DA, Wang HP, editors. Dev. Appl. NonNewtonian flows, Vol. 231: 99-105.

[23] T. Hayat, M. Khan, and M. Ayub, (2004), Couette and Poiseuille flows of an Oldroyd 6-constant fluid with magnetic field, J. Math. Anal. Appl. , Vol. 298: 225-244.

[24] T. Hayat, M. Khan, and M. Ayub, (2007), the effect of the slip condition on flows of an Oldroyd 6-constant fluid, J. Comp. Appl. Math. , Vol, 202: 402-413.

[25] T. Hayat, M. Khan, and S. Asghar, (2004), Magnetohydrodynamic flow of an Oldroyd 6-constant fluid, Appl. Math. Comp., Vol. 155: 417-425.

[26] T. Hayat, R. Ellahi, and F. M. Mahomed, (2009), Exact solution of a thin film flow of an Oldroyd 6-constant fluid over a moving belt, Comm. Nonlinear Sci. Num. Sim. , Vol. 14: 133-139.

[27] U. Farooq, T. Hayat, A. Alsaedi, and Shijun Liao, (2014), Heat and mass transfer of two-layer flows of third-grade nano-fluids in a vertical channel, Appl. Math. Comp., vol. 242: 528-540.

[28] Y. Wang, and T. Hayat, (2008), Fluctuating flow of a Maxwell fluid past a porous plate with variable suction, Nonlinear Analysis: R. W. Appl., Vol. 9: 1269-1282.

[29] Y. Wang, T. Hayat, and K. Hutter, (2004), Magnetohydrodynamic flow of an Oldroyd 8-constant fluid in a porous medium, Can. J. Phys., Vol. 82: 965-980.

[30] Y. Wang, T. Hayat, and K. Hutter, (2005), On non-linear magnetohydrodynamic problems of an Oldroyd 6-constant fluid, Int. J. NonLinear Mech. Vol. 40: 49-58. 